\title{
A discrete, geometrically exact method for simulating nonlinear, elastic and inelastic beams
}

\author{
Claire Lestringant $^{\mathrm{a}}$, Basile Audoly ${ }^{\mathrm{b}}$, Dennis Kochmann $^{\mathrm{a}, \mathrm{c}}$ \\ ${ }^{a}$ Mechanics 83 Materials, Department of Mechanical and Process Engineering, ETH Zürich, 8092 Zürich, Switzerland \\ ${ }^{b}$ Laboratoire de Mécanique des Solides, CNRS, Institut Polytechnique de Paris, 91120 Palaiseau Cedex, France \\ ${ }^{c}$ Graduate Aerospace Laboratories, California Institute of Technology, Pasadena, CA 91125, USA
}

\begin{abstract}
We present an extension of a discrete, geometrically exact beam formulation based on discrete framed curves and discrete parallel transport originally introduced in the computer graphics community. In combination with variational constitutive updates, our numerical scheme decouples the kinematics from the material behavior, and can handle finite rotations as well as a wide class of constitutive laws depending on the stretching, flexural and torsional strain and strain rates. We demonstrate its capabilities through a suite of benchmark problems involving elastic, viscous and visco-elastic beams. The method fits naturally in existing finite element frameworks and is well suited to engineering applications. It can efficiently and accurately simulate the nonlinear deformation of slender beams featuring complex material behavior, such as those found in the topical design of flexible structural metamaterials.
\end{abstract}

Keywords: nonlinear beam theory, finite element method, constitutive model, variational calculus

\section{Introduction}

Slender beams are solids with one dimension much larger than the other two. This geometry allows them to undergo large deformations even when subjected to moderate mechanical forces and moderate strains. The resulting behavior is nonlinear, as exemplified by snapping or buckling, which makes these structures promising building blocks for the design of multistable and reconfigurable metamaterials, as described e.g. in the review papers by $\mathrm{Hu}$ and Burgueño (2015); Bertoldi et al. (2017). When combined with active materials through recent additive manufacturing techniques, beams can pave the way towards engineering metamaterials with properties tunable in time (so-called 4D-printing), using e.g. thermomechanical properties of shape-memory polymers (Wagner et al., 2017; Ding et al., 2018), swelling (Gladman et al., 2016), photoactuation (Shankar et al., 2013) or magnetorheological effects (Jackson et al., 2018). Such applications highlight the need for numerical tools that can efficiently predict the mechanical response of slender structures displaying complex constitutive behaviors, such as chemo-mechanical swelling (when the structure is submerged in a fluid), magneto-mechanically coupled behavior, residual thermal stresses in polymers (Ding et al., 2018) or the rate-dependent visco-elastic behavior displayed by the polymeric materials used in most 3D-printing technologies (Bertoldi et al., 2017). This paper presents a versatile numerical model, based on one-dimensional nonlinear Cosserat theory, that can handle a wide range of constitutive models.

There is a vast amount of literature on numerical beam models in the engineering community. Geometrically exact models, initially developed by Simo (1985); Simo and Vu-Quoc (1986) and later improved and extended by Cardona and Geradin (1988); Ibrahimbegović (1995); Sonneville et al. (2014) among others, are based on strain measures that remain accurate in the presence of finite rotations. By contrast, the co-rotational formulation (Crisfield, 1990) is based on linearized strain measures and has hence been termed "non-geometrically exact" by some authors. Difficulties arise when interpolating finite rotations in geometrically exact methods, including problems associated with non-objectivity and path-dependence (Crisfield and Jelenić, 1998). These were addressed in Betsch and Steinmann (2002); Eugster et al. (2014) by switching from an interpolation of the rotations to a parametrization of the frame directors. Besides, 
the directors' rotation and the centerline's translations are usually parameterized independently, so that constraints or penalty methods are required for the simulation of unshearable beams: this tends to be numerically inefficient and/or impractical.

Here, we model discrete beams based on a method which originated from the computer graphics community (Bergou et al., 2008; Jawed et al., 2017). It is geometrically exact but, contrary to other geometrically exact methods, it embeds the unshearability constraint directly into its formulation. This allows the directors rotation to be parameterized with a minimal set of degrees of freedom (Bergou et al., 2008; Jung et al., 2011); extensions to shearable beam models are nevertheless possible (Gazzola et al., 2018).

Over the last decade, this method has been applied to a variety of applications ranging from elastic beams (Bergou et al., 2008) to inextensible elastic ribbons (Shen et al., 2015), to viscous threads (Bergou et al., 2010; Audoly et al., 2013), to visco-elastic rods (Lang et al., 2011; Linn et al., 2013), to problems including contact, self-contact and friction (Jawed et al., 2014; Kaufman et al., 2014; Gazzola et al., 2018). However, those implementations were dedicated to particular applications, and were specific to each particular type of constitutive behaviors such as linear elasticity (Bergou et al., 2008; Shen et al., 2015; Jawed et al., 2017), linear viscosity (Bergou et al., 2010; Audoly et al., 2013) or visco-elasticity (Lang et al., 2011; Linn et al., 2013). By contrast, we here propose a general approach within the framework of the finite element method (FEM), in which the geometrically exact discrete beam model's implementation is independent of the constitutive law. We adopt variational constitutive updates (Ortiz and Stainier, 1999) to treat inelastic material models: as a consequence, our beam implementation can be combined with a variety of material behaviors including, e.g., rate-dependent constitutive laws. We formulate the equations of motion in a time-discrete setting, and we introduce inelastic strain contributions as internal variables. Starting from an elastic strain energy density and a dissipation potential (Biot, 1954; Halphen and Nguyen, 1975), we formulate the incremental local kinetic law governing the evolution of internal variables. The equations of motion are derived starting from the same potentials. The discrete beam model thus provides the nonlinear kinematics, i.e., the strain measures and their variations, which can be combined with a versatile choice of potentials describing the specific constitutive law. The framework is versatile in that it decouples the extraction of discrete strain measures of slender beams from the specific material behavior, thus making a single numerical framework suitable for the use of various material models (including elastic, viscoelastic, and viscous behavior). With the kinematic framework laid out here, it will be straightforward for users in the future to adapt the implementation to other material models.

In order to lay out the unified framework, which we believe will be valuable to researchers from the engineering community, this paper includes a self-contained presentation of the geometric description from Bergou et al. (2008), including the important notions borrowed from differential geometry. We also provide examples covering three distinct constitutive models: linear elasticity, pure viscosity, and visco-elasticity. For each constitutive law, we describe the potentials used to formulate the governing equations in the discrete numerical setting as well as their continuous counterparts. After discussing the details of the implementation for solving both dynamic and quasistatic problems, we demonstrate the power of the presented method through a suite of benchmark problems, featuring instabilities, nonlinear geometric coupling between bending and twist, as well as natural curvature. Elastic, viscous and visco-elastic material models are covered, as well as quasistatic and dynamic problems. We validate the convergence of our method and show that it competes with classical nonlinear beam formulations.

Outlook. Section 2 introduces the kinematics and strain measures of a continuous beam. The notions of parallel transport and holonomy, which are central to the discretization, are introduced in Section 3. In Section 4 we detail the discretization strategy and we define the discrete beam kinematics and strain measures. Section 5 focuses on calculating the variations of the discrete strain measures at the element level, as required for the numerical implementation. We introduce several 1D constitutive models in Section 6: linear elasticity, pure viscosity, and linear visco-elasticity. In Section 7 we define the equations of motion for a discrete beam and we discuss the numerical solution of quasistatic and dynamic problems. Finally, in Section 8 we present numerical results that serve for validation and illustrate the capability of our numerical scheme. 
Notation. In the following, we use bold symbols for tensors (using lower-case characters for first-order tensors and upper-case characters for higher-order tensors), while non-bold symbols represent scalars. The dot operator $(\cdot)$ defines the contraction between the last index of the first tensor with the first index of the second tensor, no matter what the ranks of the two tensors are. For example, when applied to a second-order tensor $\mathbf{M}$ and a first-order tensor (vector) $\mathbf{v}$ it yields the vector with components $(\mathbf{M} \cdot \mathbf{v})_{i}=\sum_{k=1}^{d} M_{i k} v_{k}$ in $d$ dimensions. For two second-order tensors $\mathbf{M}$ and $\mathbf{N}$ this yields a second-order tensor with components $(\mathbf{M} \cdot \mathbf{N})_{i j}=\sum_{k=1}^{d} M_{i k} N_{k j}$. For two vectors $\mathbf{u}$ and $\mathbf{v}$ it yields the scalar dot product $\mathbf{u} \cdot \mathbf{v}=\mathbf{u} \cdot \mathbf{v}=\sum_{k=1}^{d} u_{k} v_{k}$. The dyadic product $\mathbf{u} \otimes \mathbf{v}$ of two vectors $\mathbf{u}$ and $\mathbf{v}$ is defined through the relation $(\mathbf{u} \otimes \mathbf{v}) \cdot \mathbf{w}=(\mathbf{v} \cdot \mathbf{w}) \mathbf{u}$ for any vector $\mathbf{w}$.

We denote the first variation of a function $f$ depending on a vector $\mathbf{u}$ by

$$
\delta f=\operatorname{D} f(\mathbf{u}) \cdot \delta \mathbf{u} \quad \text { where } \quad \operatorname{D} f(\mathbf{u}) \cdot \mathbf{v}=\lim _{t \rightarrow 0} \frac{f(\mathbf{u}+t \mathbf{v})-f(\mathbf{u})}{t},
$$

for any admissible vector $\mathbf{v}$. We define the second variation of $f$ as

$$
\delta^{2} f=\delta \mathbf{u}^{1} \cdot \mathrm{D}^{2} f(\mathbf{u}) \cdot \delta \mathbf{u}^{2} \quad \text { where } \quad \delta \mathbf{v}^{1} \cdot \mathrm{D}^{2} f(\mathbf{u}) \cdot \delta \mathbf{v}^{2}=\lim _{t \rightarrow 0} \frac{\mathrm{D} f\left(\mathbf{u}+t \delta \mathbf{v}^{2}\right) \cdot \delta \mathbf{v}^{1}-\mathrm{D} f(\mathbf{u}) \cdot \delta \mathbf{v}^{1}}{t}
$$

for any admissible $\delta \mathbf{v}^{1}, \delta \mathbf{v}^{2}$.

The dependence of all variables on time is implied, and we use $\dot{f}=\frac{\mathrm{d} f}{\mathrm{~d} t}$ to denote the material derivative of $f$ with respect to time. Further, a comma in indices indicates a partial derivative with respect to the subsequent variables, e.g., $f_{, \varepsilon}=\frac{\partial f}{\partial \varepsilon}$.

\section{Kinematics of a continuous beam}

\subsection{Geometry}

We describe the kinematics of a slender beam using the Lagrangian curvilinear coordinate $s$, which runs along the beam's centerline. By convention, $s$ is defined as the arc-length coordinate in an initial, undeformed configuration. The current configuration of the beam's centerline is defined by the vector field $\mathbf{r}(s)$ and we denote by $\mathbf{t}(s)=\mathbf{r}^{\prime}(s) /\left\|\mathbf{r}^{\prime}(s)\right\|$ its unit tangent, where $\mathbf{r}^{\prime}(s)=\frac{\mathrm{d} \mathbf{r}}{\mathrm{d} s}$ represents the spatial derivative. Following Cosserat theory, we capture the orientation of the beam's cross-section by introducing a set of orthonormal frame vectors $\left(\mathbf{d}_{1}(s), \mathbf{d}_{2}(s), \mathbf{d}_{3}(s)\right)=\left(\mathbf{d}_{I}(s)\right)_{I \in\{1,2,3\}}$ at every point along the beam. These frame vectors are termed material directors. We focus on unshearable beam models (which can be justified from threedimensional elasticity under fairly generic assumptions), which means that one of the directors, say $\mathbf{d}_{3}$ must remain aligned with the tangent at every point. This is captured by the kinematic constraint

$$
\mathbf{d}_{3}(s)=\mathbf{t}(s)
$$

as shown in Figure 2.1(a).

(a)

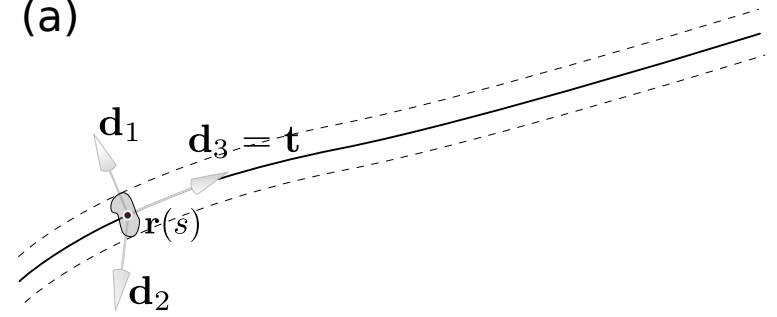

(b)

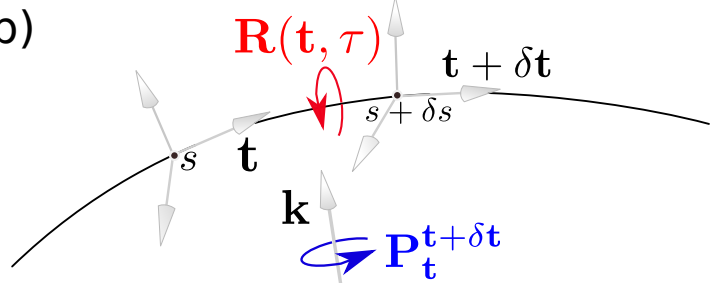

Figure 2.1: Kinematics of a continuous beam. (a) Definition of the deformed centerline $\mathbf{r}(s)$, the unit tangent $\mathbf{t}(s)$, and the material directors $\left(\mathbf{d}_{I}(s)\right)_{I \in\{1,2,3\}}$. (b) Decomposition of the flexural and torsional strains using parallel transport, illustrating Eq. (3.5). 


\subsection{Strain measures}

We define the axial strain $\varepsilon(s)$ by $\varepsilon(s)=\left\|\mathbf{r}^{\prime}(s)\right\|-1$, which shows that $\mathbf{r}^{\prime}(s)=(1+\varepsilon(s)) \mathbf{t}(s)$. Due to orthonormality, the spatial derivative of the material directors can be written as

$$
\mathbf{d}_{I}^{\prime}(s)=\boldsymbol{\Omega}(s) \times \mathbf{d}_{I}(s) \quad \text { for } \quad I \in\{1,2,3\},
$$

where the pseudo-vector $\boldsymbol{\Omega}(s)$ is called the rotation gradient (or Darboux vector in the context of differential geometry). It can be shown that the unshearability constraint (2.1) implies that $\boldsymbol{\Omega}$ is of the form $\boldsymbol{\Omega}(s)=$ $\mathbf{k}(s)+\tau(s) \mathbf{t}(s)$, where $\mathbf{k}(s)=\mathbf{t}(s) \times \mathbf{t}^{\prime}(s)$ is the so-called binormal vector, and the component of $\boldsymbol{\Omega}(s)$ along the unit tangent, $\tau(s)=\boldsymbol{\Omega}(s) \cdot \mathbf{t}(s)$, defines the (kinematic) twist. The bending strains are classically defined as the components of the binormal in the cross-sectional frame,

$$
\kappa_{I}(s)=\mathbf{k}(s) \cdot \mathbf{d}_{I}(s) \quad \text { for } \quad I \in\{1,2\} .
$$

The axial strain $\varepsilon(s)$, the twist $\tau(s)$ and the curvatures $\kappa_{1}(s)$ and $\kappa_{2}(s)$ define the four strain measures that enter into the constitutive equations for the beam, see Section 6 .

Similarly, considering the time derivative, another pseudo-vector $\boldsymbol{\pi}(s)$ is defined such that

$$
\dot{\mathbf{d}}_{I}(s)=\boldsymbol{\pi}(s) \times \mathbf{d}_{I}(s) \quad \text { for } \quad I \in\{1,2,3\},
$$

where $\boldsymbol{\pi}(s)=\mathbf{t}(s) \times \dot{\mathbf{t}}(s)+\vartheta(s) \mathbf{t}(s)$ is known as the angular velocity vector, and $\vartheta(s)$ is the spinning velocity about the tangent. For conciseness, we drop the argument $s$ in the following and tacitly assume that all kinematic measures depend on $s$.

Several technical complications arise when numerically solving the nonlinear equations for slender beams. First, the four strain measures $\varepsilon, \tau, \kappa_{1}$ and $\kappa_{2}$ depend nonlinearly on the current configuration of the beam, which involves finite rotations. Furthermore, the strain measures involve high-order derivatives of kinematic quantities with respect so $s$, since the binormal vector (which defines the bending strains) depends on $\mathbf{r}^{\prime \prime}(s)$. Finally, one has to account for the nonlinear constraint (2.1) coupling the rotations with the centerline's position. In order to overcome these difficulties, we rely on the parametrization introduced by Bergou et al. (2008, 2010), which provides a geometrically exact description of the discrete beam kinematics satisfying the nonlinear constraint (2.1) automatically. We describe this parametrization in Section 4; it relies heavily on the geometric notion of parallel transport, which is introduced next.

\section{Parallel transport and holonomy}

For two unit vectors $\mathbf{v}_{1}$ and $\mathbf{v}_{2}$ in $\mathbb{R}^{3}$, the parallel transport $\mathrm{P}_{\mathbf{v}_{1}}^{\mathbf{v}_{2}}$ is defined as the rotation about the vector $\mathbf{v}_{1} \times \mathbf{v}_{2}$ which maps $\mathbf{v}_{1}$ onto $\mathbf{v}_{2}$. It satisfies the following properties by construction:

$$
\left(\mathrm{P}_{\mathbf{v}_{1}}^{\mathbf{v}_{2}}\right)^{\mathrm{T}} \cdot \mathrm{P}_{\mathbf{v}_{1}}^{\mathbf{v}_{2}}=\mathbf{1}, \quad \mathrm{P}_{\mathbf{v}_{1}}^{\mathbf{v}_{2}} \cdot \mathbf{v}_{1}=\mathbf{v}_{2} \quad \text { and } \quad \mathrm{P}_{\mathbf{v}_{1}}^{\mathbf{v}_{2}} \cdot\left(\mathbf{v}_{1} \times \mathbf{v}_{2}\right)=\mathbf{v}_{1} \times \mathbf{v}_{2}
$$

where $\mathbf{1}$ denotes the identity. An explicit expression for the parallel transport operator (as long as $\mathbf{v}_{1} \nVdash \mathbf{v}_{2}$ ) is given by

$$
\mathrm{P}_{\mathbf{v}_{1}}^{\mathbf{v}_{2}}=\mathbf{v}_{2} \otimes \frac{\mathbf{v}_{1}-c \mathbf{v}_{2}}{s^{2}}+\left(2 c \mathbf{v}_{2}-\mathbf{v}_{1}\right) \otimes \frac{-c \mathbf{v}_{1}+\mathbf{v}_{2}}{s^{2}}+\frac{\left(\mathbf{v}_{1} \times \mathbf{v}_{2}\right) \otimes\left(\mathbf{v}_{1} \times \mathbf{v}_{2}\right)}{s^{2}},
$$

where $c=\mathbf{v}_{1} \cdot \mathbf{v}_{2}$ denotes the cosine of the angle spanned by $\left(\mathbf{v}_{1}, \mathbf{v}_{2}\right)$, and $s=\sqrt{1-c^{2}}$ its sine. Equation (3.2) can be proven easily by observing that this operator satisfies the properties (3.1) in the basis $\frac{1}{s^{2}}\left(\mathbf{v}_{1}-\right.$ $\left.c \mathbf{v}_{2},-c \mathbf{v}_{1}+\mathbf{v}_{2}\right)$ dual to $\left(\mathbf{v}_{1}, \mathbf{v}_{2}\right)$. The right-hand side of equation (3.2) is ill-defined when $\mathbf{v}_{1} \| \mathbf{v}_{2}$. When $\mathbf{v}_{2}=-\mathbf{v}_{1}$, this is because the parallel transport itself is undefined, a situation that we can avoid by selecting a small enough time step and grid size. If, however, $\mathbf{v}_{2}=\mathbf{v}_{1}$, the parallel transport is the identity, $\mathbf{1}$, and the singularity in the right-hand of (3.2) is an artifact: when $\mathbf{v}_{1} \approx \mathbf{v}_{2}$, we replace the singular expression (3.2) by its expansion

$\mathrm{P}_{\mathbf{v}_{1}}^{\mathbf{v}_{2}}=c \mathbf{1}+\left(\mathbf{v}_{1} \times \mathbf{v}_{2}\right)_{\times}+\left(\frac{1}{2}+\frac{1-c}{4}+\frac{(1-c)^{2}}{8}\left(1+\frac{\left|\mathbf{v}_{1} \times \mathbf{v}_{2}\right|^{2}}{4}\right)\right)\left(\mathbf{v}_{1} \times \mathbf{v}_{2}\right) \otimes\left(\mathbf{v}_{1} \times \mathbf{v}_{2}\right)+\mathcal{O}\left(\left(\mathbf{v}_{1}-\mathbf{v}_{2}\right)^{7}\right)$, 
where, for any vector $\mathbf{v}, \mathbf{v}_{\times}$denotes the skew-symmetric matrix such that $\mathbf{v}_{\times} \cdot \mathbf{x}=\mathbf{v} \times \mathbf{x}$. When numerically implementing parallel transport, we switch from (3.2) to the approximation (3.3) when $\left|\mathbf{v}_{1}-\mathbf{v}_{2}\right|<0.06$ (recall that $\left|\mathbf{v}_{1}\right|=\left|\mathbf{v}_{2}\right|=1$ ) in order to ensure that the numerical error made by replacing (3.2) by (3.3) remains smaller than $10^{-10}$ in absolute value.

For future reference, we note that a truncated version of the expansion (3.3) can be written as

$$
\mathrm{P}_{\mathbf{v}}^{\mathbf{v}}+\delta \mathbf{v}=\mathbf{1}+(\mathbf{v} \times \delta \mathbf{v})_{\times}+\mathcal{O}\left(\delta \mathbf{v}^{2}\right)
$$

where both $\mathbf{v}$ and $\mathbf{v}+\delta \mathrm{v}$ are unit vectors.

\subsection{Spatial parallel transport and kinematic twist}

A key observation is that parallel transport provides an intrinsic way to measure the kinematic twist $\tau$. Expanding (2.2) for a small increment $\delta s$ of the curvilinear coordinate $s$ yields $\mathbf{d}_{i}(s+\delta s)=\left(\mathbf{1}+\boldsymbol{\Omega}_{\times} \delta s\right)$. $\mathbf{d}_{i}(s)+\mathcal{O}\left(\delta s^{2}\right)=\left(\mathbf{1}+\mathbf{k}_{\times} \delta s+\tau \mathbf{t}_{\times} \delta s\right) \cdot \mathbf{d}_{i}(s)+\mathcal{O}\left(\delta s^{2}\right)=\left(\mathbf{1}+(\mathbf{t} \times \delta \mathbf{t})_{\times}+\tau \delta s \mathbf{t}_{\times}\right) \cdot \mathbf{d}_{i}(s)+\mathcal{O}\left(\delta s^{2}\right)$, where $\delta \mathbf{t}$ denotes the increment of the unit tangent vector $\mathbf{t}$ associated with the increment $\delta s$. This expansion can be re-written as $\mathbf{d}_{i}(s+\delta s)=\left(\mathbf{1}+(\mathbf{t} \times \delta \mathbf{t})_{\times}\right) \cdot\left(\mathbf{1}+\tau \delta s \mathbf{t}_{\times}\right) \cdot \mathbf{d}_{i}(s)+\mathcal{O}\left(\delta s^{2}\right)$. By identifying $\left(\mathbf{1}+(\mathbf{t} \times \delta \mathbf{t})_{\times}\right)$as an infinitesimal parallel transport from equation (3.4), we can decompose the rotation mapping the directors frame $\mathbf{d}_{i}(s)$ to a neighboring frame $\mathbf{d}_{i}(s+\delta s)$ into a twisting rotation and parallel transport,

$$
\mathbf{d}_{i}(s+\delta s)=\mathbf{P}_{\mathbf{t}(s)}^{\mathbf{t}(s+\delta s)} \cdot \mathbf{R}(\mathbf{t}(s), \tau(s) \delta s) \cdot \mathbf{d}_{i}(s)+\mathcal{O}\left(\delta s^{2}\right),
$$

where $\mathbf{R}(\mathbf{u}, \beta) \in \mathrm{SO}(3)$ denotes the rotation by angle $\beta$ about unit vector $\mathbf{u}$. This decomposition is depicted in Figure 2.1(b): twisting is defined geometrically by relying on parallel transport. A discrete version of this decomposition will play a key role in the discrete beam model.

\subsection{Temporal parallel transport and spinning velocity}

Carrying out a similar expansion starting from equation (2.3) for a small time increment $\delta t$, we obtain

$$
\mathbf{d}_{i}(t+\delta t)=\mathbf{P}_{\mathbf{t}(t)}^{\mathbf{t}(t+\delta t)} \cdot \mathbf{R}(\mathbf{t}(t), \vartheta(t) \delta t) \cdot \mathbf{d}_{i}(t)+\mathcal{O}\left(\delta t^{2}\right),
$$

where we have dropped the dependency on the curvilinear coordinate $s$ for the sake of brevity.

\subsection{Holonomy}

Given a set of unit vectors $\left(\mathbf{v}_{1}, \mathbf{v}_{2}, \cdots, \mathbf{v}_{m}\right)$, consider the composition of parallel transports along the closed loop, namely $\mathbf{P}_{\mathbf{v}_{m}}^{\mathbf{v}_{1}} \cdot \mathbf{P}_{\mathbf{v}_{m-1}}^{\mathbf{v}_{m}} \cdot \ldots \cdot \mathbf{P}_{\mathbf{v}_{2}}^{\mathbf{v}_{3}} \cdot \mathbf{P}_{\mathbf{v}_{1}}^{\mathbf{v}_{2}}$. It is easy to show that this is a rotation which leaves $\mathbf{v}_{1}$ invariant: it is a rotation about the axis spanned by $\mathbf{v}_{1}$. Denoting as $\mathcal{A}\left(\mathbf{v}_{1}, \mathbf{v}_{2}, \cdots, \mathbf{v}_{m}\right)$ its angle, we have

$$
\mathbf{P}_{\mathbf{v}_{m}}^{\mathbf{v}_{1}} \cdot \mathbf{P}_{\mathbf{v}_{m-1}}^{\mathbf{v}_{m}} \cdots \mathbf{P}_{\mathbf{v}_{2}}^{\mathbf{v}_{3}} \cdot \mathbf{P}_{\mathbf{v}_{1}}^{\mathbf{v}_{2}} \cdot=\mathbf{R}\left(\mathbf{v}_{1}, \mathcal{A}\left(\mathbf{v}_{1}, \mathbf{v}_{2}, \cdots, \mathbf{v}_{m}\right)\right) \text {. }
$$

The quantity $\mathcal{A}\left(\mathbf{v}_{1}, \mathbf{v}_{2}, \cdots, \mathbf{v}_{m}\right)$ is called the holonomy in differential geometry. The Fuller-White-Calugareanu theorem (Fuller (1971, 1978); Aldinger et al. (1995); de Vries (2005)) states that the holonomy is equal to the signed spherical area of the polygon spanned by the $n$ vectors $\left(\mathbf{v}_{1}, \mathbf{v}_{2}, \cdots, \mathbf{v}_{m}\right)$ on the unit sphere, also termed spherical excess or solid angle (see also Jawed et al. (2017), Chapter 7).

The case $m=4$ will be of particular interest in the following. Since the spherical quadrangle spanned by $\left(\mathbf{v}_{1}, \mathbf{v}_{2}, \mathbf{v}_{3}, \mathbf{v}_{4}\right)$ on the unit sphere can be split into two triangles, one has

$$
\mathcal{A}\left(\mathbf{v}_{1}, \mathbf{v}_{2}, \mathbf{v}_{3}, \mathbf{v}_{4}\right)=\mathcal{A}\left(\mathbf{v}_{1}, \mathbf{v}_{2}, \mathbf{v}_{4}\right)+\mathcal{A}\left(\mathbf{v}_{4}, \mathbf{v}_{2}, \mathbf{v}_{3}\right) .
$$

In order to calculate the holonomy $\mathcal{A}\left(\mathbf{v}_{1}, \mathbf{v}_{2}, \mathbf{v}_{3}, \mathbf{v}_{4}\right)$, it is thus sufficient to calculate the signed area of spherical triangles on the unit sphere. An explicit method for the latter is proposed in Appendix A. 


\section{Discrete strain measures}

In our implementation, we adopt an updated-Lagrangian approach in order to accurately capture large rotations. We call reference configuration the known configuration at the start of the current timestep (at time $t$ ), and current configuration that at the end of the timestep (at time $t+\delta t$ ). Starting from the reference configuration, we use temporal parallel transport to define the current state of the directors; this warrants that the stiffness matrices are sparse (and in fact banded, when the beam has the topology of a segment). By contrast, spatial parallel transport was used in the original implementation of Bergou et al. (2008), which has the drawback of making the stiffness matrices full.

\subsection{Discrete parametrization of rotations}

The centerline of a beam is described by a discrete line represented by the $n$ vertices labelled from 0 to $n-1$ as $\left\{\left(\mathbf{x}_{0}\right)_{\star}, \cdots,\left(\mathbf{x}_{n-1}\right)_{\star}\right\}$ in the reference configuration and $\left\{\mathbf{x}_{0}, \cdots, \mathbf{x}_{n-1}\right\}$ in the current configuration. In the sequel, we identify by the asterisk ${ }_{\star}$ ) all quantities pertaining to the reference configuration. By contrast, quantities carrying no asterisk are defined in the current configuration.

In both configurations, each pair of adjacent vertices $\left(\mathbf{x}_{i}, \mathbf{x}_{i+1}\right)$ for $i \in\{0, n-2\}$ spans a segment of index $i$, as depicted in Figure (4.1), which is described by the vector $\mathbf{e}^{i}=\mathbf{x}_{i+1}-\mathbf{x}_{i}\left(\right.$ resp. $\left.\mathbf{e}_{\star}^{i}\right)$. For each segment, we define the associated unit vector $\mathbf{t}^{i}=\mathbf{e}^{i} /\left\|\mathbf{e}^{i}\right\|$ (analogously $\mathbf{t}_{\star}^{i}=\mathbf{e}_{\star}^{i} /\left\|\mathbf{e}_{\star}^{i}\right\|$ ) and the material frame $\left(\mathbf{d}^{i}\right)_{I, I=1,2,3}$ with $\mathbf{d}_{3}^{i}=\mathbf{t}^{i}$ (analogously $\left(\mathbf{d}_{\star}^{i}\right)_{I, I=1,2,3}$ with $\left(\mathbf{d}_{3}^{i}\right)_{\star}=\mathbf{t}_{\star}^{i}$ ). In the following, segment-based quantities are labelled with superscripts while vertex-based quanties are labelled with subscripts.

We observe that the composition of the rotation that maps the reference frame $\left(\mathbf{d}_{\star}^{i}\right)_{I, I=1,2,3}$ into its current configuration $\left(\mathbf{d}^{i}\right)_{I, I=1,2,3}$ (applied first) with the parallel transport $\mathbf{P}_{\mathbf{t}^{i}}^{\mathbf{t}_{\star}^{i}}=\left(\mathbf{P}_{\mathbf{t}_{\star}^{i}}^{\mathbf{t}^{i}}\right)^{T}$ that brings back $\mathbf{t}^{i}$ to $\mathbf{t}_{\star}^{i}$ (applied second) leaves $\mathbf{t}_{\star}^{i}$ invariant, and is therefore a rotation about $\mathbf{t}_{\star}^{i}$. Denoting by $\omega^{i}$ its angle, we have

$$
\mathbf{d}_{I}^{i}=\mathbf{P}_{\mathbf{t}_{\star}^{i}}^{\mathbf{t}^{i}} \cdot \mathbf{R}\left(\mathbf{t}_{\star}^{i}, \omega^{i}\right) \cdot\left(\mathbf{d}_{I}^{i}\right)_{\star} \quad \text { for } \quad I \in\{1,2,3\} .
$$

The equation above is analogous to equation (3.6) and the angle $\omega^{i}$ is identified to the integral over the time step of the spinning velocity, $\omega^{i} \approx \vartheta \delta t$.

Equation (4.1) defines the material frame $\left(\mathbf{d}^{i}\right)_{I, I=1,2,3}$ in terms of $\mathbf{t}^{i}$ (which itself is a function of the position of the adjacent vertices) and of a scalar degree of freedom $\omega^{i}$. As noted by Bergou et al. (2008), this parameterization has the advantage of incorporating the unshearability condition. Being based on temporal parallel transport, this variant offers the additional benefit that the stiffness matrix will be sparse (usually banded), since the rotation is parameterized by local degrees of freedom only (adjacent nodes position, plus local rotation $\left.\omega^{i}\right)$.

Overall, the current configuration is parameterized by the global vector of degrees of freedom

$$
\mathbf{u}=\left(\mathbf{x}_{0}, \omega^{0}, \cdots, \mathbf{x}_{i-1}, \omega^{i-1}, \mathbf{x}_{i}, \omega^{i}, \mathbf{x}_{i+1}, \cdots, \omega^{n-2}, \mathbf{x}_{n-1}\right) .
$$

It also depends on the reference configuration, although this will be implicit in our notation. For a 3D problem, $\mathbf{u} \in \mathbb{R}^{4 n-1}$. In the $2 \mathrm{D}$ case, i. e., when the beam is restricted to a plane, the rotational degrees of freedom $\omega^{i}$ must remain zero, so that $\mathbf{u} \in \mathbb{R}^{2 n}$ effectively. Since the $2 \mathrm{D}$ model can be viewed as a particular case of the 3D model with some degrees of freedom frozen, we will focus on the latter in the sequel.

Following an updated-Lagrangian approach, the reference configuration is updated to be the current configuration after each converged simulation (load or time) step. It is important to note that this reference configuration differs from the initial, undeformed configuration used to initialize, e.g., the undeformed segment lengths and volumes at the start of the simulation.

In the following section, we show how a discrete measure of the twist $\tau$ can be defined, first, and calculated in terms of the degrees of freedom $\mathbf{u}$, next. 


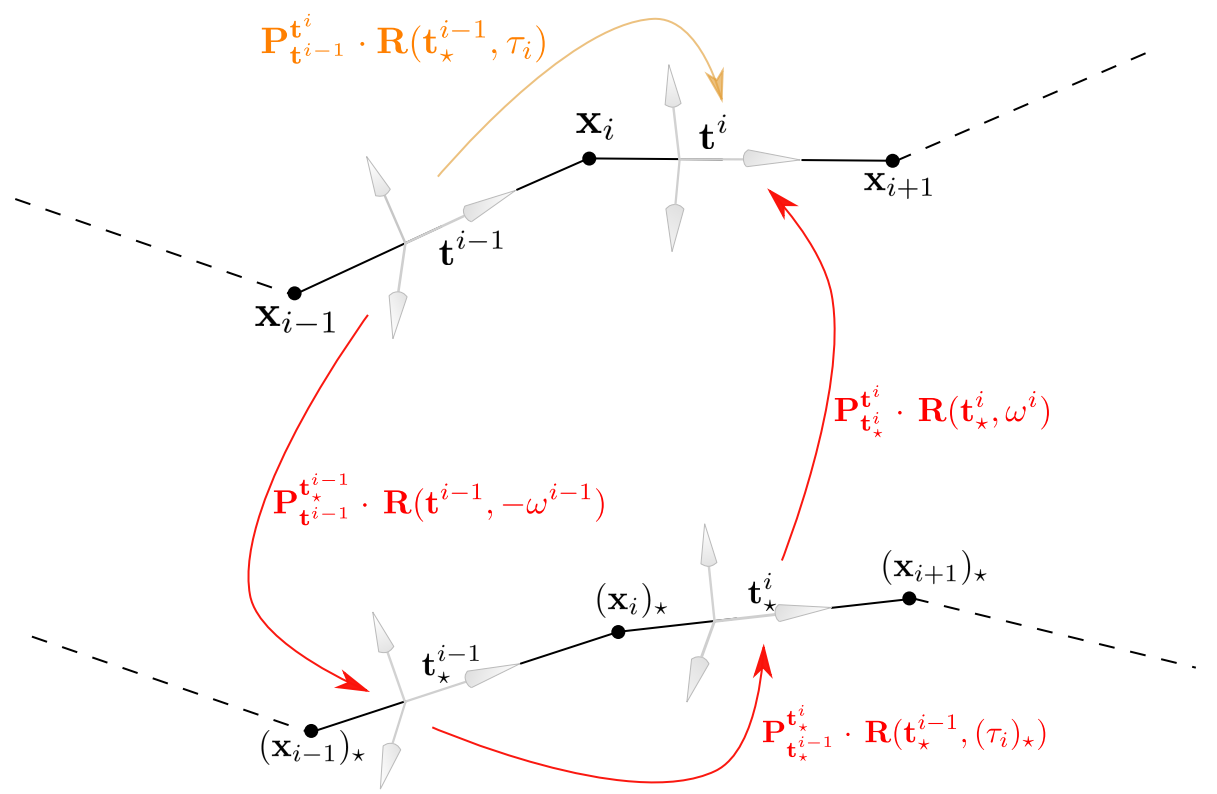

Figure 4.1: Commutative diagram for evaluating the discrete twist $\tau_{i}$. Portion $\left\{\mathbf{x}_{i-1}, \mathbf{x}_{i}, \mathbf{x}_{i+1}\right\}$ of a discrete beam in the reference configuration (bottom) and in the current configuration (top). As shown by equation (4.4), the rotation mapping $\left(\mathbf{d}^{i-1}\right)_{I, I \in\{1,2,3\}}$ into $\left(\mathbf{d}^{i}\right)_{I, I \in\{1,2,3\}}$ can be described equivalently by combining parallel transport in space and twist in the current configuration (orange arrow and left-hand side of the equation) or by combining two parallel transports in time between current and reference configurations, one parallel transport in space in the reference configuration, and a rotation about the axis in the undeformed configuration (red arrows and right-hand side of the equation).

\subsection{Definition and calculation of the discrete twist}

We consider a portion of the discrete line representing the centerline of a beam, centered at an interior vertex $i(i \in\{1, \cdots, n-2\})$ and defined by three vertices $\left\{\mathbf{x}_{i-1}, \mathbf{x}_{i}, \mathbf{x}_{i+1}\right\}$ in the current configuration (respectively, $\left\{\left(\mathbf{x}_{i-1}\right)_{\star},\left(\mathbf{x}_{i}\right)_{\star},\left(\mathbf{x}_{i+1}\right)_{\star}\right\}$ in the reference configuration), as depicted in Figure 4.1.

Considering the rotation that maps the frame $\left(\mathbf{d}^{i-1}\right)_{I, I \in\{1,2,3\}}$ onto $\left(\mathbf{d}^{i}\right)_{I, I \in\{1,2,3\}}$ and using a decomposition similar to (4.1), we have

$$
\mathbf{d}_{I}^{i}=\mathbf{P}_{\mathbf{t}^{i-1}}^{\mathbf{t}^{i}} \cdot \mathbf{R}\left(\mathbf{t}^{i-1}, \tau_{i}\right) \cdot \mathbf{d}_{I}^{i-1} \quad \text { for } \quad I \in\{1,2,3\}
$$

where the angle $\tau_{i}$ denotes the discrete twist associated with the vertex $i$ in the current configuration.

By inserting the parameterization of the frames $\left(\mathbf{d}^{i-1}\right)_{I, I=1,2,3}$ and $\left(\mathbf{d}^{i}\right)_{I, I=1,2,3}$ in terms of $\omega^{i-1}$ and $\omega^{i}$ from the previous section, we can arrive at an expression for the discrete twisting strain $\tau_{i}$ in terms of the degrees of freedom: this is the goal of the remainder of this section. Note the difference with the original work of Bergou et al. (2008), where $\tau_{i}$ was used as a degree of freedom and the above equation (which relies on spatial parallel transport) was used to parameterize the frame of directors, with the consequence that stiffness matrices were full. The approach followed here has been employed in subsequent work (Bergou et al., 2010; Audoly et al., 2013).

An equation analogous to (4.3) but applying to the reference configuration can be obtained as $\left(\mathbf{d}_{I}^{i}\right)_{\star}=$ $\mathbf{P}_{\mathbf{t}_{\star}^{i-1}}^{\mathbf{t}_{\star}^{i}} \cdot \mathbf{R}\left(\mathbf{t}_{\star}^{i-1},\left(\tau_{i}\right)_{\star}\right) \cdot\left(\mathbf{d}_{I}^{i-1}\right)_{\star}$, where $\left(\tau_{i}\right)_{\star}$ is the twisting strain in the reference configuration, which is known from the previous time step. Now, eliminating $\left(\mathbf{d}^{i-1}\right)_{I, I \in\{1,2,3\}}$ and $\left(\mathbf{d}^{i}\right)_{I, I \in\{1,2,3\}}$ from equation (4.3) using equation (4.1) twice yields

$$
\mathbf{P}_{\mathbf{t}^{i-1}}^{\mathbf{t}^{i}} \cdot \mathbf{R}\left(\mathbf{t}^{i-1}, \tau_{i}\right)=\mathbf{P}_{\mathbf{t}_{\star}^{i}}^{\mathbf{t}^{i}} \cdot \mathbf{R}\left(\mathbf{t}_{\star}^{i}, \omega^{i}\right) \cdot \mathbf{P}_{\mathbf{t}_{\star}^{i-1}}^{\mathbf{t}_{\star}^{i}} \cdot \mathbf{R}\left(\mathbf{t}_{\star}^{i-1},\left(\tau_{i}\right)_{\star}\right) \cdot \mathbf{P}_{\mathbf{t}^{i-1}}^{\mathbf{t}_{\star}^{i-1}} \cdot \mathbf{R}\left(\mathbf{t}^{i-1},-\omega^{i-1}\right) .
$$

This equation is interpreted graphically as a commutative diagram in Figure 4.1, as explained in the legend. 
This relation can be simplified using the conjugacy property $\mathbf{P}_{\mathbf{u}}^{\mathbf{v}} \cdot \mathbf{R}(\mathbf{u}, \beta)=\mathbf{R}(\mathbf{v}, \beta) \cdot \mathbf{P}_{\mathbf{u}}^{\mathbf{v}}$ valid for any set of unit vectors $\mathbf{u}$ and $\mathbf{v}$ and for any angle $\beta$. This yields

$$
\mathbf{R}\left(\mathbf{t}_{\star}^{i-1}, \gamma_{i}\right)=\mathbf{P}_{\mathbf{t}^{i-1}}^{\mathbf{t}_{\star}^{i-1}} \cdot\left(\mathbf{P}_{\mathbf{t}^{i-1}}^{\mathbf{t}^{i}}\right)^{T} \cdot \mathbf{P}_{\mathbf{t}_{\star}^{i}}^{\mathbf{t}^{i}} \cdot \mathbf{P}_{\mathbf{t}_{\star}^{i-1}}^{\mathbf{t}_{\star}^{i}}
$$

where $\gamma_{i}=\left(\tau_{i}-\left(\tau_{i}\right)_{\star}\right)-\left(\omega^{i}-\omega^{i-1}\right)$, that is

$$
\tau_{i}=\left(\tau_{i}\right)_{\star}+\left(\omega^{i}-\omega^{i-1}\right)+\gamma_{i}
$$

In the right-hand side of (4.5), $\left(\mathbf{P}_{\mathbf{t}^{i-1}}^{\mathbf{t}^{i}}\right)^{T}=\mathbf{P}_{\mathbf{t}^{i}}^{\mathbf{t}^{i-1}}$, which shows that this right-hand is a combination of parallel transports along a closed loop drawn on the unit sphere. Using the result established in Section 3.3, we conclude that $\gamma_{i}$ can be calculated as the sum of the signed area of two spherical triangles

$$
\gamma_{i}=\mathcal{A}\left(\mathbf{t}_{\star}^{i-1}, \mathbf{t}_{\star}^{i}, \mathbf{t}^{i-1}\right)+\mathcal{A}\left(\mathbf{t}^{i-1}, \mathbf{t}_{\star}^{i}, \mathbf{t}^{i}\right),
$$

where the spherical triangle area can be found using the method given in Appendix A. Note that, like the area of a spherical triangle, the holonomy and the parameter $\gamma_{i}$ are defined modulo $2 \pi$ : assuming sufficiently small load increments and time steps, we use the determination of the parameter $\gamma_{i}$ which is the smallest in absolute value.

The current unit tangents $\mathbf{t}^{i-1}$ and $\mathbf{t}^{i}$ can be reconstructed from the positions of the adjacent nodes: equations (4.6) and (4.7) provide an explicit expression of the twist $\tau_{i}$ in the current configuration in terms of the degrees of freedom $\mathbf{x}_{i-1}, \mathbf{x}_{i}, \mathbf{x}_{i+1}$ (on which $\mathbf{t}^{i-1}$ and $\mathbf{t}^{i}$ depend), $\omega^{i-1}$ and $\omega^{i}$.

\subsection{Expressions for the discrete strains}

Having obtained an explicit expression for the twist $\tau_{i}$ in Eqs. (4.6-4.7), we complement the kinematic description of a discrete beam by providing expressions for the axial and bending strains. The discrete axial strain in segment $i$ is defined via the change of length of the segment vector $\mathbf{e}^{i}$, viz.

$$
\varepsilon^{i}=\frac{\left\|\mathbf{e}^{i}\right\|^{2}-\left(l_{0}^{i}\right)^{2}}{2\left(l_{0}^{i}\right)^{2}},
$$

where $l_{0}^{i}$ denotes the initial, undeformed length of segment $i$. We define the bending strains as

$$
\kappa_{i}^{I}=\mathbf{k}_{i} \cdot\left[\frac{\mathbf{d}_{I}^{i-1}+\mathbf{d}_{I}^{i}}{2}\right] \text { for } \quad I \in\{1,2\},
$$

where $\mathbf{k}_{i}$ denotes the vertex-based discrete curvature binormal,

$$
\mathbf{k}_{i}=\frac{2 \mathbf{t}^{i-1} \times \mathbf{t}^{i}}{1+\mathbf{t}^{i-1} \cdot \mathbf{t}^{i}}
$$

The twisting (4.6) and bending strains (4.9) are defined at interior vertices while the axial strain defined in (4.8) is defined at every segment. These discrete strain measures for bending and twist are the discrete counterparts of the continuous strains introduced in Section 2.2, and the local value of the continuous axial strain introduced in Section 2.2 can be recovered using $\varepsilon^{i} \approx\left((1+\varepsilon)^{2}-1\right) / 2$. Many other discrete stretching and bending strains are consistent with the continuous strain measures, and could be used alternatively.

Note that the vertex-based strain measures defined above are integrated quantities: the local values of the continuous counterparts can be recovered as $\kappa_{i}^{I} /\left(\tilde{l}_{i}\right)_{0}$ for the discrete curvature in direction $I(I \in\{1,2\})$ and $\tau_{i} /\left(\tilde{l}_{i}\right)_{0}$ for the discrete twist, where

$$
\tilde{l}_{i}=\frac{l^{i-1}+l^{i}}{2}
$$

denotes the Voronoi length associated with the vertex of index $i$, and $l^{i-1}$ and $l^{i}$ are the current lengths of the two segments adjacent to this vertex.

This framework can be extended to model shearable beams by relaxing the constraint $\mathbf{t}^{i}=\mathbf{d}_{3}^{i}$ and considering an additional shear strain measure, such as $\mu_{1}^{i}=\mathbf{t}^{i} \cdot \mathbf{d}_{1}^{i}$ and $\mu_{2}^{i}=\mathbf{t}^{i} \cdot \mathbf{d}_{2}^{i}$. As it does not imply any major difficulty, see Gazzola et al. (2018), this extension will not be discussed further here. 


\subsection{Summary of the geometric beam description}

Table 1 summarizes all the geometric quantities involved in the calculation of the discrete strain, and mentions the place where they are defined. In particular, the material frame vectors and the axial strain are defined at every segment, while the twist, the holonomy and the bending strains are defined at interior vertices. Anticipating on the modelling of inelastic materials in Section 6, we introduce a set of additional, internal variables $\mathbf{z}_{l}$ defined at interior vertices.

Table 1: Geometry of a discrete beam

Algorithm 1 below outlines how the geometry of a discrete beam is initialized and updated at the different steps of a dynamic or a quasistatic simulation. In this algorithm, we use indices $i, k, l$ to iterate over vertices, segments and interior vertices, respectively, in accord with the conventions used in Table 1. More details on the methods and equations solved during a load or time step are provided in Section 7 and summarized in Algorithm 2. Note that $\omega^{k}$ denotes an increment of angle per timestep: we introduce the associated angular velocity $\theta^{k}$. With this notation, we define the vectors of velocities and accelerations along a discrete beam, respectively, as

$$
\begin{aligned}
& \mathbf{v}=\left(\dot{\mathbf{x}}_{0}, \theta^{0}, \cdots, \dot{\mathbf{x}}_{i-1}, \theta^{i-1}, \dot{\mathbf{x}}_{i}, \theta^{i}, \dot{\mathbf{x}}_{i+1}, \cdots, \theta^{n-2}, \dot{\mathbf{x}}_{n-1}\right), \\
& \mathbf{a}=\left(\ddot{\mathbf{x}}_{0}, \dot{\theta}^{0}, \cdots, \ddot{\mathbf{x}}_{i-1}, \dot{\theta}^{i-1}, \ddot{\mathbf{x}}_{i}, \dot{\theta}^{i}, \ddot{\mathbf{x}}_{i+1}, \cdots, \dot{\theta}^{n-2}, \ddot{\mathbf{x}}_{n-1}\right) .
\end{aligned}
$$

Details on the calculation of velocities and accelerations in a dynamic scheme are provided in Section 7.1.

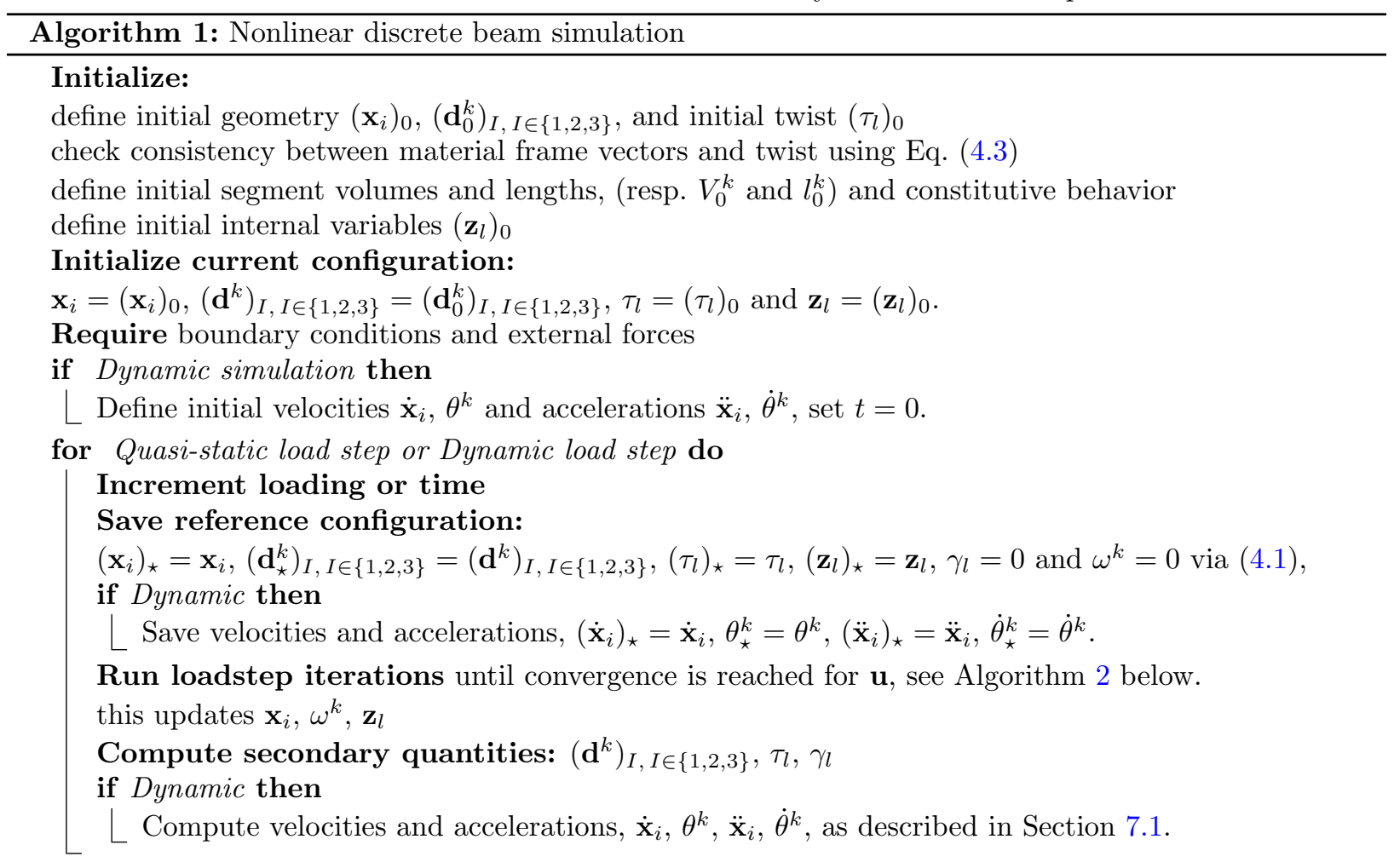

In the next section, we define the discrete beam elements and we detail the calculation of the variations of the discrete strains at the element level. This complements the description of the geometric part of the numerical formulation. 


\section{Discrete strains and their variations at the element's level}

\subsection{Discrete beam elements}

In our implementation, a portion of a discrete curve spanned by the two segments $i-1$ and $i$ (i.e., defined by the three vertices $\mathbf{x}_{i-1}, \mathbf{x}_{i}$ and $\mathbf{x}_{i+1}$, using the labeling conventions from Figure 4.1) defines the discrete beam element of index $i$. The discretization (4.2) thus defines $n-2$ elements, one per interior vertex. The local degrees of freedom associated with the element of index $i$ (for $i \in\{1, \cdots, n-2\}$ ) are defined as

$$
\mathbf{u}_{i}=\left(\mathbf{x}_{i-1}, \mathbf{x}_{i}, \mathbf{x}_{i+1}, \omega^{i-1}, \omega^{i}\right) .
$$

This vector of local degrees of freedom is built by copying a few selected values from the global vector of degrees of freedom $\mathbf{u}$. Mathematically, this can be represented as a multiplication by the connectivity matrix $\mathbf{C}_{i}$, whose coefficients are either 0 or 1 ,

$$
\mathbf{u}_{i}=\mathbf{C}_{i} \cdot \mathbf{u} .
$$

We introduce the vector of strains of element $i$ as

$$
\mathbf{E}_{i}\left(\mathbf{u}_{i}\right)=\left(\varepsilon^{i-1}, \varepsilon^{i}, \kappa_{i}^{1}, \kappa_{i}^{2}, \tau_{i}\right)
$$

where the discrete axial, bending and twisting strains have been defined in Eqs. (4.8), (4.9) and (4.6), respectively. Note that we include in the element strain $\mathbf{E}_{i}$ the axial strains $\varepsilon^{i-1}$ and $\varepsilon^{i}$ from the two segments $\mathbf{e}^{i-1}$ and $\mathbf{e}^{i}$ that are part of the element. This is because we chose to introduce a single type of finite element, that handles both the bending/twisting effects (which takes place at interior vertices) and the stretching effect (which takes place at segments); to be able to calculate the latter, the element needs to be informed about the axial strain of both segments participating into the element. An alternative design choice, explored in previous papers such as that of Bergou et al. (2008) is to have one type of element for bending and stretching, similar to the above, and a different type of element for stretching (whose stencil is two adjacent nodes). Note that dealing with special boundary conditions, such as clamping at boundary or rigid connections between beams in a truss, might require specific elements, such as rigid body elements for the junctions and specific clamped elements including a rigid constraint on the rotations of a segment; this is a consequence of the low-order interpolation introduced by the discretization. In this paper, we focus on the modeling of individual beams. Finally, we store a set of internal variables $\mathbf{z}_{i}$ on each element $(i \in\{1, \cdots, n-2\})$, which store, e.g., the viscous strains, as will be discussed in Section 6 .

With our choice of parametrization, the material directors admit a local support. This implies that the strain vectors $\mathbf{E}_{i}$ is a function of the local the degrees of freedom $\mathbf{u}_{i}$ in the element $i$. In order to derive the residual force vector and stiffness matrix of the discrete beam formulation, we consider an infinitesimal perturbation of local degrees of freedom,

$$
\delta \mathbf{u}_{i}=\left(\delta \mathbf{x}_{i-1}, \delta \mathbf{x}_{i}, \delta \mathbf{x}_{i+1}, \delta \omega^{i-1}, \delta \omega^{i}\right),
$$

resulting from a perturbation of the vertex positions $\mathbf{x}_{i-1}, \mathbf{x}_{i}$ and $\mathbf{x}_{i+1}$ and of segments' angles $\omega^{i-1}$ and $\omega^{i}$. To avoid duplicate derivations, we use in the following the generic index $i \pm$ to refer to the segment on the left-hand side $i-=i-1$, or that on the right-hand side, $i+=i$.

With a view to calculating the internal forces and the consistent tangent matrix used in the numerical scheme, we proceed to provide explicit expressions for the first variation of the various geometric quantities involved in the calculation of the strains (see Table 1), viz. segment and unit tangent vectors, holonomy and material frame vectors, and the binormal vector, respectively. The first variations of the axial, bending and twisting strains are provided in Sections 5.5, 5.6 and 5.7. In Section 5.8 we discuss the calculation of the second variations of the strain measures, whose detailed expressions are provided in Appendix B.

We note that, since all strain measures and derived quantities depend on the current value of the local displacement vector $\mathbf{u}_{i}$, in the following we drop the explicit dependence on $\mathbf{u}_{i}$ and simply write the gradient as $\mathrm{D} f\left(\mathbf{u}_{i}\right)=\mathrm{D} f$ for the sake of brevity. 


\subsection{First variation of the segment vectors and of the unit tangents}

The variation of segment vector $i-1$ (respectively $i$ ) is deduced from definition (5.1). It writes

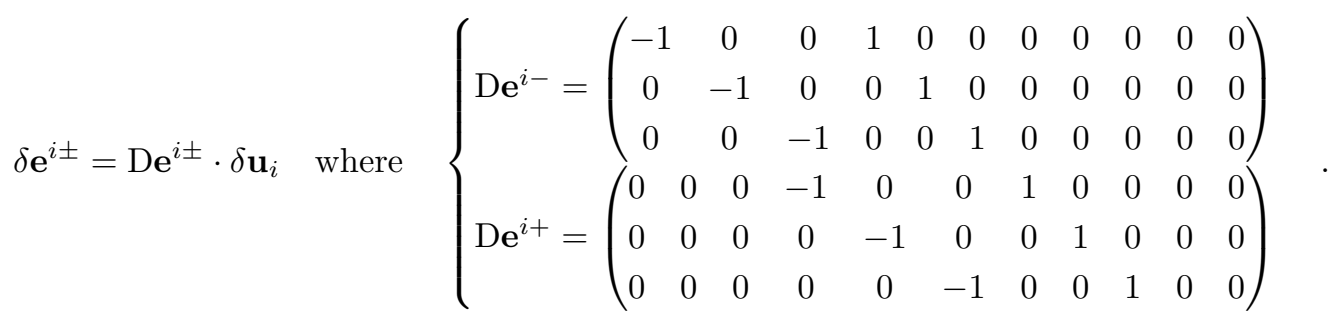

Using this relation, we obtain the first variation of the associated unit vector $\mathbf{t}^{i \pm}=\frac{\mathbf{e}^{i \pm}}{\left\|\mathbf{e}^{i \pm}\right\|}$ as

$$
\delta \mathbf{t}^{i \pm}=\mathrm{Dt}^{i \pm} \cdot \delta \mathbf{u}_{i} \quad \text { where } \quad \mathrm{Dt}^{i \pm}=\frac{\mathbf{1}-\mathbf{t}^{i \pm} \otimes \mathbf{t}^{i \pm}}{\left\|\mathbf{e}^{i \pm}\right\|} \cdot \mathrm{De}^{i \pm} .
$$

\subsection{First variation of the holonomy and of the material frame vectors}

Using the expression for the first variation of the signed area of a spherical triangle defined in Appendix A.3 and the definition (4.7), we express the first variation of the holonomy $\gamma_{i}$ as

$$
\delta \gamma_{i}=\mathrm{D} \gamma_{i} \cdot \delta \mathbf{u}_{i} \quad \text { where } \quad \mathrm{D} \gamma_{i}=\frac{\mathbf{k}_{i}+\mathbf{b}_{\star}^{i-1}}{2} \cdot \mathrm{Dt} \mathbf{t}^{i-1}+\frac{\mathbf{k}_{i}-\mathbf{b}_{\star}^{i}}{2} \cdot \mathrm{Dt} \mathbf{t}^{i},
$$

where $\mathrm{Dt}^{i \pm}$ have just been found in equation (5.4). In this equation, $\mathbf{b}_{\star}^{i-1}=\mathbf{b}\left(\mathbf{t}_{\star}^{i-1}, \mathbf{t}^{i-1}\right), \mathbf{b}_{\star}^{i}=\mathbf{b}\left(\mathbf{t}_{\star}^{i}, \mathbf{t}^{i}\right)$ are the reference and current binormals associated with temporal parallel transport, and $\mathbf{b}(\mathbf{u}, \mathbf{v})$ is the function defined by

$$
\mathbf{b}\left(\mathbf{v}_{1}, \mathbf{v}_{2}\right)=\frac{2 \mathbf{v}_{1} \times \mathbf{v}_{2}}{1+\mathbf{v}_{1} \cdot \mathbf{v}_{2}}
$$

for any pair of unit vectors $\mathbf{v}_{1}$ and $\mathbf{v}_{2}$.

The variation of the material directors $\left(\mathbf{d}^{i \pm}\right)_{I, I \in\{1,2,3\}}$ are obtained from definition (4.1) as

$$
\mathbf{d}_{I}^{i \pm}+\delta \mathbf{d}_{I}^{i \pm}=\mathbf{P}_{\mathbf{t}_{\star}^{i \pm}}^{\mathbf{t}^{i \pm}+\delta \mathbf{t}^{i \pm}} \cdot \mathbf{R}\left(\mathbf{t}_{\star}^{i \pm}, \omega^{i \pm}+\delta \omega^{i \pm}\right) \cdot\left(\mathbf{d}_{I}^{i \pm}\right)_{\star} \cdot
$$

We introduce the holonomy along the path $\left(\mathbf{t}_{\star}^{i \pm}, \mathbf{t}^{i \pm}+\delta \mathbf{t}^{i \pm}, \mathbf{t}^{i \pm}\right)$ as $\delta \phi^{i \pm}=\mathcal{A}\left(\mathbf{t}_{\star}^{i \pm}, \mathbf{t}^{i \pm}+\delta \mathbf{t}^{i \pm}, \mathbf{t}^{i \pm}\right)$. It satisfies by definition $\mathbf{P}_{\mathbf{t}_{\star}^{i \pm}}^{\mathbf{t}^{i \pm}+\delta \mathbf{t}^{i \pm}}=\mathbf{P}_{\mathbf{t}^{i \pm}}^{\mathbf{t}^{i \pm}+\delta \mathbf{t}^{i \pm}} \cdot \mathbf{P}_{\mathbf{t}_{\star}^{i \pm}}^{\mathbf{t}^{i \pm}} \cdot \mathbf{R}\left(\mathbf{t}_{\star}^{i \pm}, \delta \phi^{i \pm}\right)$. By inserting into the equation above, we obtain

$$
\begin{aligned}
\mathbf{d}_{I}^{i \pm}+\delta \mathbf{d}_{I}^{i \pm} & =\mathbf{P}_{\mathbf{t}^{i \pm}}^{\mathbf{t}^{i \pm}+\delta \mathbf{t}^{i \pm}} \cdot \mathbf{R}\left(\mathbf{t}^{i \pm}, \delta \phi^{i \pm}+\delta \omega^{i \pm}\right) \cdot \mathbf{d}_{I}^{i \pm} \\
& =\left[\mathbf{1}+\left(\mathbf{t}^{i \pm} \times \delta \mathbf{t}^{i \pm}\right)_{\times}\right] \cdot\left[\mathbf{1}+\left(\delta \phi^{i \pm}+\delta \omega^{i \pm}\right) \mathbf{t}_{\times}^{i \pm}\right] \cdot \mathbf{d}_{I}^{i \pm}
\end{aligned}
$$

Next, we insert the expression for the variation of holonomy $\delta \phi^{i \pm}$ from Appendix A, and obtain

$$
\delta \mathbf{d}_{I}^{i \pm}=\operatorname{Dd}_{I}^{i \pm} \cdot \delta \mathbf{u}_{i} \quad \text { where } \quad \operatorname{Dd}_{I}^{i \pm}=\sum_{J=1}^{2} \eta_{I J} \mathbf{d}_{J}^{i \pm} \otimes \boldsymbol{\zeta}^{i \pm}-\left[\sum_{J=1}^{2} \eta_{I J} \mathbf{d}_{J}^{j} \otimes \frac{\mathbf{b}_{\star}^{i \pm}}{2}+\mathbf{t}^{i \pm} \otimes \mathbf{d}_{J}^{i \pm}\right] \cdot \mathrm{Dt}^{i \pm}
$$

for $I \in\{1,2\}$, where $\boldsymbol{\zeta}^{i \pm}=\left(0, \cdots 0, \delta_{(i-1) i \pm}, \delta_{i i \pm}\right)$, such that $\boldsymbol{\zeta}^{i \pm} \cdot \delta \mathbf{u}_{i}=\delta \omega^{i \pm}$, and $\eta_{I J}$ denotes the antisymmetric symbol, $\eta_{11}=\eta_{22}=0, \eta_{12}=1$ and $\eta_{21}=-1$. The first variations of the unit tangents Dt ${ }^{i \pm}$ have been obtained in $(5.4)$ 


\subsection{First variation of a binormal vector}

In order to compute the variation of the bending strains, we first express the variation of the function $\mathbf{b}\left(\mathbf{v}_{1}, \mathbf{v}_{2}\right)$ defined in (5.6): for any infinitesimal variation of the two unit vectors $\left[\delta \mathbf{v}_{1}, \delta \mathbf{v}_{2}\right]$,

$$
\delta \mathbf{b}=\mathrm{Db}\left(\mathbf{v}_{1}, \mathbf{v}_{2}\right) \cdot\left[\delta \mathbf{v}_{1}, \delta \mathbf{v}_{2}\right]=\mathrm{D}_{1} \mathbf{b}\left(\mathbf{v}_{1}, \mathbf{v}_{2}\right) \cdot \delta \mathbf{v}_{1}+\mathrm{D}_{2} \mathbf{b}\left(\mathbf{v}_{1}, \mathbf{v}_{2}\right) \cdot \delta \mathbf{v}_{2},
$$

where the gradients with respect to the first and second argument read, respectively,

$$
\mathrm{D}_{1} \mathbf{b}\left(\mathbf{v}_{1}, \mathbf{v}_{2}\right)=-2\left[\frac{\left(\mathbf{v}_{2}\right)_{\times}}{1+\mathbf{v}_{1} \cdot \mathbf{v}_{2}}+\frac{\left(\mathbf{v}_{1} \times \mathbf{v}_{2}\right) \otimes \mathbf{v}_{2}}{\left(1+\mathbf{v}_{1} \cdot \mathbf{v}_{2}\right)^{2}}\right], \quad \mathrm{D}_{2} \mathbf{b}\left(\mathbf{v}_{1}, \mathbf{v}_{2}\right)=2\left[\frac{\left(\mathbf{v}_{1}\right)_{\times}}{1+\mathbf{v}_{1} \cdot \mathbf{v}_{2}}-\frac{\left(\mathbf{v}_{1} \times \mathbf{v}_{2}\right) \otimes \mathbf{v}_{1}}{\left(1+\mathbf{v}_{1} \cdot \mathbf{v}_{2}\right)^{2}}\right] .
$$

Using this notation, we write the first variation of the binormal vector $\mathbf{k}_{i}$ defined in (4.10) as $\mathbf{k}_{i}=\mathbf{b}\left(\mathbf{t}^{i-1}, \mathbf{t}^{i}\right)$ in the form

$$
\delta \mathbf{k}_{i}=\underbrace{\mathrm{D}_{1} \mathbf{b}\left(\mathbf{t}^{i-1}, \mathbf{t}^{i}\right)}_{\mathrm{D}_{1} \mathbf{k}_{i}} \cdot \delta \mathbf{t}^{i-1}+\underbrace{\mathrm{D}_{2} \mathbf{b}\left(\mathbf{t}^{i-1}, \mathbf{t}^{i}\right)}_{\mathrm{D}_{\mathbf{2}} \mathbf{k}_{i}} \cdot \delta \mathbf{t}^{i}
$$

where the first variations of the unit segment vectors $\mathrm{Dt}^{i \pm}$ are given by (5.4). We now have all ingredients to compute the first variations of the axial, bending and twisting strains defined over the element $i$.

\subsection{First variation of the axial strain}

The variation of the stretch $\varepsilon^{i \pm}$ is obtained from the definition (4.8) and writes

$$
\delta \varepsilon^{i \pm}=\mathrm{D} \varepsilon^{i \pm} \cdot \delta \mathbf{u}_{i} \quad \text { where } \quad \mathrm{D} \varepsilon^{i \pm}=\frac{\mathbf{e}^{i \pm} \cdot \mathrm{D} \mathbf{e}^{i \pm}}{\left(l_{0}^{i}\right)^{2}}
$$

where $\mathrm{De}^{i \pm}$ has been obtained in Eq. (5.3).

\subsection{First variation of the bending strain}

The first variation of the bending strains is derived from the definition (4.9) using the expression for the variation of the binormal vector (5.9) and the variation of the material frame vectors (5.7). This yields

$$
\delta \kappa_{i}^{I}=\mathrm{D} \kappa_{i}^{I} \cdot \delta \mathbf{u}_{i} \quad \text { with } \quad \mathrm{D} \kappa_{i}^{I}=\frac{\mathbf{d}_{I}^{i-1}+\mathbf{d}_{I}^{i}}{2} \cdot\left(\mathrm{D}_{1} \mathbf{k}_{i} \cdot \mathrm{D} \mathbf{t}^{i-1}+\mathrm{D}_{2} \mathbf{k}_{i} \cdot \mathrm{D} \mathbf{t}^{i}\right)+\frac{\mathbf{k}_{i}}{2} \cdot\left(\mathrm{Dd}_{I}^{i-1}+\mathrm{Dd}_{I}^{i}\right),
$$

for $I \in\{1,2\}$. The gradients of the unit tangent $\mathrm{Dt}^{i \pm}$ is given by (5.4).

\subsection{First variation of the twist}

We use the definition of the discrete twist in Eq. (4.6) and the expression for the first variation of $\gamma_{i}$ from (5.5) to express the first variation of the twist as

$$
\delta \tau_{i}=\mathrm{D} \tau_{i} \cdot \delta \mathbf{u}_{i} \quad \text { where } \quad \mathrm{D} \tau_{i}=\boldsymbol{\Delta}+\frac{1}{2}\left[\left(\mathbf{b}_{\star}^{i-1}+\mathbf{k}_{i}\right) \cdot \mathrm{Dt} \mathbf{t}^{i-1}+\left(-\mathbf{b}_{\star}^{i}+\mathbf{k}_{i}\right) \cdot \mathrm{D} \mathbf{t}^{i}\right],
$$

where $\boldsymbol{\Delta}=(0, \cdots 0,-1,+1)$, such that $\boldsymbol{\Delta} \cdot \delta \mathbf{u}_{i}=\delta \omega^{i}-\delta \omega^{i-1}$, and where the first variations of the unit segment vectors $\mathrm{Dt}^{i \pm}$ are given by (5.4).

\subsection{Second variations of strains}

The second variations of all these quantities are required for the calculation of the stiffness matrices. They follow from similar calculations. For example, from (5.10) we derive the second variation of the stretch as

$$
\delta^{2} \varepsilon^{i \pm}=\delta \mathbf{u}_{i}^{1} \cdot \mathrm{D}^{2} \varepsilon^{i \pm} \cdot \mathbf{u}_{i}^{2} \quad \text { where } \quad \mathrm{D}^{2} \varepsilon^{i \pm}=\frac{\left(\mathrm{D} \boldsymbol{e}^{i \pm}\right)^{T} \cdot \mathrm{D} \boldsymbol{e}^{i \pm}}{\left(l_{0}^{i}\right)^{2}}
$$

in terms of the first variations of the unit tangents $\mathrm{Dt}^{i \pm}$ found earlier by (5.4). The second variation of the bending and twisting strains are deduced from Eqs. (5.11) and (5.12) and are given in Appendix B. 


\section{Constitutive laws}

By contrast with previous implementations of the discrete beam method, the kinematic modeling of the beam (which involves the calculation of the discrete strains and of their variations) is independent from the material behavior in our implementation. We demonstrate the applicability of our approach to slender beams made of elastic, viscous and visco-elastic materials. These three cases are discussed successively in this section. The extension to other inelastic behaviors such as plasticity, visco-plasticity or damage is straightforward and will not be discussed.

\subsection{Inextensible, elastic beam}

The elastic energy of a beam of length $L$ made of a linear elastic material is given by the classical Euler-Bernoulli model which writes, see for example Audoly and Pomeau (2010),

$$
W(\mathbf{E})=\frac{1}{2} \int_{0}^{L}\left(E A \varepsilon^{2}+E\left(I_{1} \kappa_{1}^{2}+I_{2} \kappa_{2}^{2}\right)+\mu J \tau^{2}\right) \mathrm{d} s
$$

where $\mathbf{E}=\left(\varepsilon, \kappa_{1}, \kappa_{2}, \tau\right)$ denotes the vector of strains, $E$ is Young's modulus, $\mu$ is the shear modulus, $A$ the cross-sectional area, $I_{1}$ and $I_{2}$ the geometric moments of inertia, and $J$ is the geometric torsion constant (or torsional rigidity). In our examples, we assume that the strain remains small (in particular, this assumes $|\varepsilon| \ll 1$ ), so that any variation in the coefficients $A, I_{1}, I_{2}$ and $J$ can be ignored: the elastic constitutive law is linear.

To model a beam made of a linear elastic material, the energy (6.1) is approximated by $W_{\text {discrete }}=$ $\sum_{i=0}^{n-2} W_{i}$ where $W_{i}$ is the elastic energy of a discrete beam element defined as

$$
W_{i}\left(\mathbf{E}_{i}\right)=\frac{1}{2}\left[\frac{E A}{2}\left(l_{0}^{i-1}\left(\varepsilon^{i-1}\right)^{2}+l_{0}^{i}\left(\varepsilon^{i}\right)^{2}\right)+\left(\tilde{l}_{i}\right)_{0} E I_{1}\left(\tilde{\kappa}_{i}^{1}\right)^{2}+\left(\tilde{l}_{i}\right)_{0} E I_{2}\left(\tilde{\kappa}_{i}^{2}\right)^{2}+\left(\tilde{l}_{i}\right)_{0} \mu J \tilde{\tau}_{i}^{2}\right],
$$

where $\left(\tilde{l}_{i}\right)_{0}$ denotes the Voronoi length of element $i$ in the undeformed configuration, cf. (4.11). In this expression, the tilde denotes that the strain measures have been rescaled by $\left(\tilde{l}_{i}\right)_{0}: \tilde{\kappa}_{i}^{I}=\kappa_{i}^{I} /\left(\tilde{l}_{i}\right)_{0}$ for $I \in\{1,2\}$ and $\tilde{\tau}_{i}=\tau_{i} /\left(\tilde{l}_{i}\right)_{0}$, these quantities converge to the continuous measures of strain $\left(\kappa_{i}^{I}\right.$ and $\tau_{i}$, as defined in $(4.9)$ and (4.6)) in the limit of a zero segment length, as discussed in Section 4.3. In our numerical examples with elastic beams, we consider weakly extensible beam (i.e. $E A$ is large enough) so that the strain $|\varepsilon| \ll 1$.

\subsection{Viscous beam with surface tension}

The one-dimensional constitutive law of a thin thread having a circular cross-section and made of an incompressible Newtonian fluid with dynamic viscosity $\eta$ is captured by the Rayleigh potential (Audoly et al., 2013)

$$
\mathcal{D}(\dot{\mathbf{E}})=\frac{\eta}{2} \int_{0}^{L} \frac{1}{(1+\varepsilon)}\left[3 A(\varepsilon) \dot{\varepsilon}^{2}+3 I(\varepsilon)\left(\dot{\kappa}_{1}^{2}+\dot{\kappa}_{2}^{2}\right)+2 I(\varepsilon) \dot{\tau}^{2}\right] \mathrm{d} s,
$$

where $A(\varepsilon)$ and $I(\varepsilon)$ denote the current values of the cross-sectional area and area moment of inertia. The fluid being incompressible, we have $A(\varepsilon)=A_{0} /(1+\varepsilon)$ and $I(\varepsilon)=I_{0} /(1+\varepsilon)^{2}$. The factor $1 /(1+\varepsilon)$ in the integral comes from a coefficient $(1+\varepsilon)$ in front of $\mathrm{d} s$ which makes appear the current arc length, and a factor $1 /(1+\varepsilon)^{2}$ in front of the (Lagrangian) strain rates squared, which makes appear the Eulerian strain rates squared, see Audoly et al. (2013) for details (equations (28-29)). Surface tension is represented by a potential proportional to the area of the lateral surface,

$$
W(\varepsilon)=\gamma \int_{0}^{L} 2 \pi r(\varepsilon)(1+\varepsilon) \mathrm{d} s
$$

where $\gamma$ is the surface tension and $r(\varepsilon)=\sqrt{A(\varepsilon) / \pi}$ the current radius of the thread. 


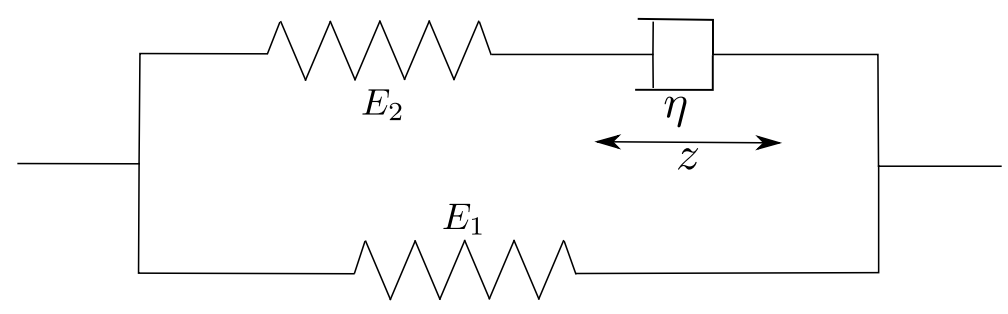

Figure 6.1: Standard visco-elastic behavior.

The discrete viscous dissipation potential representing (6.3) is $\mathcal{D}_{\text {discrete }}=\sum_{i=0}^{n-2} \mathcal{D}_{i}$ with

$$
\mathcal{D}_{i}\left(\dot{\mathbf{E}}_{i}\right)=\frac{\eta}{2}\left[\frac{3}{2}\left(V^{i-1}\left(\frac{l_{0}^{i-1}}{l_{\star}^{i-1}}\right)^{4}\left(\dot{\varepsilon}^{i-1}\right)^{2}+V^{i}\left(\frac{l_{0}^{i}}{l_{\star}^{i}}\right)^{4}\left(\dot{\varepsilon}^{i}\right)^{2}\right)+\frac{3\left(\tilde{I}_{i}\right)_{\star}\left(\dot{\boldsymbol{\kappa}}^{i}\right)^{2}}{\left(\tilde{l}_{i}\right)_{\star}}+\frac{2\left(\tilde{I}_{i}\right)_{\star} \dot{\tau}^{2}}{\left(\tilde{l}_{i}\right)_{\star}}\right],
$$

where we used the fact that the cross-sectional area of the fluid cylinder associated with segment $i$ can be written as $A_{\star}^{i}=V^{i} / l_{\star}^{i}$ and thus $A /(1+\varepsilon)$ can be recovered as $V^{i} l_{0}^{i} /\left(l_{\star}^{i}\right)^{2}$. Note that with our definition of the discrete axial strain $\varepsilon^{i}$ in (4.8), the local value of the continuous axial strain rate $\dot{\varepsilon}$ on segment $i$ is recovered as $l_{0}^{i} \dot{\varepsilon}^{i} / l_{\star}^{i}$. We define $V^{i}$ as the fluid volume in segment $i$, which is a fixed quantity since the fluid is incompressible. In this expression, $\left(\tilde{l}_{i}\right)_{\star}$ is the Voronoi length of element $i$ in the reference configuration and the geometric moment of inertia of element $i$ in the reference configuration is defined as

$$
\left(\tilde{I}_{i}\right)_{\star}=\frac{1}{4 \pi}\left[\frac{\left(V^{i} / l_{\star}^{i}\right)^{2}+\left(V^{i-1} / l_{\star}^{i-1}\right)^{2}}{2}\right],
$$

where we used the fact that the squared radius of the fluid cylinder associated with segment $i$ can be written as $\left(r_{\star}^{i}\right)^{2}=\left(V^{i} / l_{\star}^{i}\right) / \pi$. Following the discretization of (6.4) introduced in Audoly et al. (2013), we write the discrete surface energy for an element of index $i$ as

$$
W_{i}\left(\mathbf{E}_{i}\right)=2 \gamma \frac{\sqrt{\pi V^{i-1}\left(l_{0}\right)^{i-1} \sqrt{1+2 \varepsilon^{i}-1}}+\sqrt{\pi V^{i}\left(l^{i}\right)_{0} \sqrt{1+2 \varepsilon^{i}}}}{2},
$$

where we have used the fact that the lateral surface of the fluid cylinder associated with segment $i$ can be written as $\left(2 \pi r^{i}\right) l^{i}=2 \sqrt{\pi^{2}\left(r^{i}\right)^{2}\left(l^{i}\right)^{2}}=2 \sqrt{\pi V^{i} l^{i}}=2 \sqrt{\pi V^{i}\left(l^{i}\right)_{0} \sqrt{1+2 \varepsilon^{i}}}$ (with our definition of the discrete axial strain, the continuous axial strain on segment $i$ is recovered as $1+\varepsilon \approx \sqrt{2 \varepsilon^{i}+1}$ ).

\subsection{Visco-elastic beam}

We also consider a beam made of a linear visco-elastic material. The uniaxial behavior of the viscoelastic material is modeled by the constitutive law of a standard linear solid with elastic moduli $E_{1}, E_{2}$ and viscosity $\eta$, as sketched in Figure 6.1. This constitutive law is identical to the one used in Gomez et al. (2019) to model the visco-elastic snap-through of a slender beam and whose results will serve for validation in our numerical examples. It corresponds to a $1 \mathrm{D}$ potential energy density and a $1 \mathrm{D}$ dissipation potential, given by, respectively,

$$
W(\mathbf{E}, z)=\frac{1}{2} \int_{0}^{L}\left[E A \varepsilon^{2}+E_{2} I_{1}\left(\kappa_{1}-z\right)^{2}+E_{1} I_{1} \kappa_{1}^{2}\right] \mathrm{d} s, \quad \mathcal{D}(\dot{z})=\frac{\eta I_{1}}{2} \int_{0}^{L} \dot{z}^{2} \mathrm{~d} s,
$$

where $z$ is an internal variable representing a permanent change of reference curvature in the viscous term, represented by the extension of the dashpot in the figure. In Eq. (6.8), we ignore the viscous effect associated with the axial strain: in our numerical example in Section 8.3, we simulate the deformation of a 2D shallow arch that is almost inextensible. The beam deformation is restricted to a plane by means of the constraints 
$\kappa_{2}=0$ and $\tau=0$. The extension to general constitutive laws including viscous axial strain, bending in the second direction, or twist is straightforward.

A discrete counterpart of the constitutive behavior (6.7) can be achieved using the following potential energy $W_{i}$ and dissipation potential $\mathcal{D}_{i}$ per element,

$$
W_{i}\left(\mathbf{E}_{i}, z_{i}\right)=\frac{E A}{4}\left(l_{0}^{i-1}\left(\varepsilon^{i-1}\right)^{2}+l_{0}^{i}\left(\varepsilon^{i}\right)^{2}\right)+\frac{I_{1}\left(\tilde{l}_{i}\right)_{0}}{2}\left(E_{1}\left(\tilde{\kappa}_{i}^{1}\right)^{2}+E_{2}\left(\tilde{\kappa}_{i}^{1}-\tilde{z}_{i}\right)^{2}\right), \quad \mathcal{D}_{i}\left(\dot{z}_{i}\right)=\frac{\eta I_{1}}{2\left(\tilde{l}^{i}\right)_{0}} \dot{\tilde{z}}_{i}^{2},
$$

where $\tilde{z}_{i}=z_{i} /\left(\tilde{l}_{i}\right)_{0}$. Here, the internal variable $\mathbf{z}_{i}=\left(z_{i}\right)$ is a scalar representing the permanent change of reference (integrated) curvature in element $i$.

\section{Equations of motion and methods of solution}

We proceed to formulate the governing equations in a discrete setting, for conservative material models in Section 7.2 and for dissipative models in Section 7.3. We discuss both equilibrium and dynamic problems. In all our forthcoming examples involving inertia, we consider homogeneous beams of mass density $\rho$ with small axial strains $|\varepsilon| \ll 1$, so that their cross-sectional area $A=A_{0}$ and geometric moment of inertia $I=I_{0}$ are constant. The kinetic energy of a beam thus writes

$$
\mathcal{E}_{c}(\dot{\boldsymbol{r}}, \vartheta)=\frac{\rho}{2} \int_{0}^{L}\left[A_{0} \dot{\boldsymbol{r}}^{2}+I_{0} \vartheta^{2}\right] \mathrm{d} s
$$

The kinetic energy (7.1) is discretized in space as $\mathcal{E}_{c}^{\text {discrete }}=\sum_{i=1}^{n-2} \mathbf{v} \cdot \mathbf{C}_{i}^{T} \cdot \mathbf{M}_{i} \cdot \mathbf{C}_{i} \cdot \mathbf{v}$ where $\mathbf{M}_{i}$ denotes the mass matrix of element $i$ and $\mathbf{v}$ is the generalized velocity defined in (4.12). Different discretizations yielding the consistent and lumped mass matrices are presented in Appendix C.

\subsection{Time discretization}

We introduce a generic time discretization as follows. We denote by $t_{0}=0$ the time in the initial, undeformed configuration, by $t_{\star}$ the time at the start of the current time step, and by $t=t_{\star}+\Delta t$ the time at the end of the current time step. Any specific finite difference scheme provides a method to compute the vectors of velocities $\mathbf{v}(4.12)$ and accelerations $\mathbf{a}(4.13)$ at the end of the current time step $t_{\star}+\Delta t$ as

$$
\mathbf{v}_{\Delta t}=\mathcal{F}\left(\mathbf{u}, \mathbf{u}_{\star}, \mathbf{v}_{\star}\right), \quad \mathbf{a}_{\Delta t}=\mathcal{G}\left(\mathbf{u}, \mathbf{u}_{\star}, \mathbf{v}_{\star}, \mathbf{a}_{\star}\right),
$$

where $\mathbf{u}_{\star}, \mathbf{v}_{\star}$ and $\mathbf{a}_{\star}$ are the vectors of degrees of freedom, velocities and accelerations at $t_{\star}$, respectively, and $\mathbf{u}$ is the vector of the degrees of freedom at $t_{\star}+\Delta t$. The functions $\mathcal{F}$ and $\mathcal{G}$ reflect the choice of finite difference discretization.

For example, an implicit Newmark scheme with average acceleration makes use, for the translational degrees of freedom, of $\mathbf{x}_{i}=\left(\mathbf{x}_{i}\right)_{\star}+\Delta t\left(\dot{\mathbf{x}}_{i}\right)_{\star}+\frac{\Delta t^{2}}{4}\left(\ddot{\mathbf{x}}_{i}+\left(\ddot{\mathbf{x}}_{i}\right)_{\star}\right)$ and $\dot{\mathbf{x}}_{i}=\left(\dot{\mathbf{x}}_{i}\right)_{\star}+\frac{\Delta t}{2}\left(\ddot{\mathbf{x}}_{i}+\left(\ddot{\mathbf{x}}_{i}\right)_{\star}\right)$, for $i \in$ $\{0, \cdots, n-1\}$. Solving the first equation for $\ddot{\mathbf{x}}_{i}$ yields

$$
\ddot{\mathbf{x}}_{i}=\frac{4}{\Delta t^{2}}\left[\mathbf{x}_{i}-\left(\mathbf{x}_{i}\right)_{\star}-\Delta t\left(\dot{\mathbf{x}}_{i}\right)_{\star}\right]-\left(\ddot{\mathbf{x}}_{i}\right)_{\star} .
$$

Inserting (7.3) into the equation for $\dot{\mathbf{x}}_{i}$ yields

$$
\dot{\mathbf{x}}_{i}=-\left(\dot{\mathbf{x}}_{i}\right)_{\star}+\frac{2}{\Delta t}\left[\mathbf{x}_{i}-\left(\mathbf{x}_{i}\right)_{\star}\right] .
$$

Applying an analogous scheme to angular velocities $\theta^{k}(k \in\{0, \cdots n-2\})$ complements the scheme with

$$
\begin{gathered}
\dot{\theta}^{k}=\frac{4}{\Delta t^{2}}\left[\omega^{k}-\Delta t \theta_{\star}^{k}\right]-\dot{\theta}_{\star}^{k}, \\
\theta^{k}=-\theta_{\star}^{k}+\frac{2}{\Delta t} \omega^{k} .
\end{gathered}
$$

Eqs. (7.4) and (7.6) provide an update rule for the velocities v: their right-hand sides can be identified with the function $\mathcal{F}_{\text {Newmark }}$ appearing in (7.2). Similarly, the right-hand sides of (7.3) and (7.5) provide a specific update rule $\mathcal{G}_{\text {Newmark }}$ that fits the general form $(7.2)$. 


\subsection{Elastic case}

In the case of an elastic beam, the elastic energy $W_{i}\left(\mathbf{E}_{i}\right)$ of the element with index $i(i \in\{1, \cdots, n-2\})$ is defined in (6.2), where $\mathbf{E}_{i}$ denotes the element's vector of strains as defined by (5.2). The vector $\boldsymbol{\Sigma}_{i}$ of internal stress in element $i$ (normal force, bending and twisting moments) writes

$$
\boldsymbol{\Sigma}_{i}\left(\mathbf{E}_{i}\right)=\left(\frac{\partial W_{i}}{\partial \varepsilon^{i-1}}, \frac{\partial W_{i}}{\partial \varepsilon^{i}}, \frac{\partial W_{i}}{\partial \kappa_{i}^{1}}, \frac{\partial W_{i}}{\partial \kappa_{i}^{2}}, \frac{\partial W_{i}}{\partial \tau_{i}}\right)
$$

We also define the vector $\mathrm{DE}_{i}$ of the first variation of the discrete strains (5.2) as

$$
\mathrm{D} \mathbf{E}_{i}=\left(\mathrm{D} \varepsilon^{i-1}, \mathrm{D} \varepsilon^{i}, \mathrm{D} \kappa_{i}^{1}, \mathrm{D} \kappa_{i}^{2}, \mathrm{D} \tau_{i}\right)
$$

where the expressions for $\mathrm{D} \varepsilon^{i \pm}, \mathrm{D} \kappa_{i}^{1}, \mathrm{D} \kappa_{i}^{2}, \mathrm{D} \tau_{i}$ are given in Eqs. (5.10), (5.11) and (5.12), respectively. As mentionned earlier, gradients without an explicit argument, such as $\mathrm{DE}_{i}$, are with respect to the local degrees of freedom $\mathbf{u}_{i}$.

We introduce a basis of $\mathbb{R}^{4 n-1}$ denoted by $\left(\tilde{\mathbf{u}}^{k}\right)_{k \in\{1, \cdots, 4 n-1\}}$ and defined as

$$
\tilde{\mathbf{u}}^{1}=(1,0,0, \cdots, 0), \quad \tilde{\mathbf{u}}^{2}=(0,1,0, \cdots, 0), \quad \cdots, \quad \tilde{\mathbf{u}}^{4 n-1}=(0,0,0, \cdots, 1) .
$$

Upon projection onto this basis, the equations of motion (obtained, e.g. by the Euler-Lagrange equations expressing the condition of stationary action) read

$$
\sum_{i=1}^{n-2}\left[\mathbf{a}_{\Delta t} \cdot \mathbf{C}_{i}^{T} \cdot \mathbf{M}_{i}+\boldsymbol{\Sigma}_{i} \cdot \mathrm{D} \mathbf{E}_{i}\right] \cdot \mathbf{C}_{i} \cdot \tilde{\mathbf{u}}^{k}-F_{\mathrm{ext}}^{k}=0
$$

for $k \in\{1, \cdots, 4 n-1\}$. Here, the acceleration $\mathbf{a}_{\Delta t}$ is discretized in time using a finite-difference scheme (i.e. we use $\mathbf{a}_{\Delta t}=\mathcal{G}\left(\mathbf{u}, \mathbf{u}_{\star}, \mathbf{v}_{\star}, \mathbf{a}_{\star}\right)$ from (7.2)), and we denote by $F_{\text {ext }}^{k}$ the external forces dual to the degree of freedom of index $k$. The discrete external force $\mathbf{F}_{\text {ext }}=\left(F_{\text {ext }}^{k}\right)_{k \in\{1, \cdots, 4 n-1\}}$ can represent actual forces applied at vertices (they are then conjugate to the corresponding translational degrees of freedom) or twisting moments (which are conjugate to the rotational degrees of freedom $\omega^{k}$ ). A bending moment (i.e., a moment whose axis is perpendicular to the centerline) can be obtained by applying two opposite, point-like forces on neighboring vertices; an example will be given in Section 8.1.

Finally, by inserting the expressions given by (7.7), (7.8), (5.10), (5.11) and (5.12) into (7.9) we obtain a set of $4 n-1$ equations for the degrees of freedom at the end of the time step $\mathbf{u}\left(t_{\star}+\Delta t\right.$ ) (on which the generalized acceleration $\mathbf{a}_{\Delta t}=\mathcal{G}\left(\mathbf{u}, \mathbf{u}_{\star}, \mathbf{v}_{\star}, \mathbf{a}_{\star}\right)$ depends). This evolution problem, akin to a second-order ordinary differential equation, requires admissible initial conditions on $\mathbf{u}, \mathbf{v}$ and $\mathbf{a}$ to be specified at $t_{0}$. The velocities and accelerations are updated at the end of each time step using, e.g. (7.4), (7.3), (7.6) and (7.5).

For quasistatic problems, the inertia term in (7.9) is omitted and the problem reduces to a set of nonlinear equations for $\mathbf{u}$

$$
\sum_{i=1}^{n-2} \boldsymbol{\Sigma}_{i} \cdot \mathrm{DE}_{i} \cdot \mathbf{C}_{i} \cdot \tilde{\mathbf{u}}^{k}-F_{\mathrm{ext}}^{k}=0,
$$

which we solved numerically using a Newton-Raphson method. Note that both $\boldsymbol{\Sigma}_{i}$ and DE $\mathbf{E}_{i}$ depend (albeit implicitly in our notation) on the unknown $\mathbf{u}$; this makes the problem nonlinear. Examples of solution of (7.10) will be presented in Section 8.1 for various types of loading.

The iterative solution schemes for both the quasistatic and implicit dynamic problems require the calculation of the stiffness matrix $\mathbf{T}$. We denote by $\mathbf{F}_{\text {int }}$ the resultant of internal stress appearing in both the dynamic equation (7.9) and in the equilibrium equation (7.10): restoring the implicit arguments,

$$
\left.\mathbf{F}_{\text {int }}(\mathbf{u})=\sum_{i=1}^{n-2} \boldsymbol{\Sigma}_{i}\left(\mathbf{E}_{i}\left(\mathbf{C}_{i} \cdot \mathbf{u}\right)\right) \cdot \mathrm{DE}_{i}\left(\mathbf{C}_{i} \cdot \mathbf{u}\right)\right) \cdot \mathbf{C}_{i} .
$$


The stiffness matrix is the gradient of internal stress resultant $\mathbf{F}_{\text {int }}(\mathbf{u})$ with respect to $\mathbf{u}:$ for $(k, l) \in$ $\{0,1, \cdots, 4 n-1\} \times\{0,1, \cdots, 4 n-1\}$, the stiffness component associated with global degrees of freedom $k$ and $l$ reads

$$
T_{k l}=\sum_{i=1}^{n-2}\left(\mathbf{C}_{i} \cdot \tilde{\mathbf{u}}^{k}\right) \cdot\left(\mathrm{DE} \mathbf{E}_{i} \cdot \mathbf{K}_{i} \cdot \mathrm{D} \mathbf{E}_{i}\right) \cdot\left(\mathbf{C}_{i} \cdot \tilde{\mathbf{u}}^{l}\right)+\boldsymbol{\Sigma}_{i} \cdot\left(\left(\mathbf{C}_{i} \cdot \tilde{\mathbf{u}}^{k}\right) \cdot \mathrm{D}^{2} \mathbf{E}_{i} \cdot\left(\mathbf{C}_{i} \cdot \tilde{\mathbf{u}}^{l}\right)\right),
$$

where $\mathrm{DE}_{i}$ denotes the first gradient of the strains as defined by Eq. (7.8), and $\mathrm{D}^{2} \mathbf{E}_{i}$ is the second gradient derived in Section 5.8 and Appendix B,

$$
\mathrm{D}^{2} \mathbf{E}_{i}=\left(\mathrm{D}^{2} \varepsilon^{i-1}, \mathrm{D}^{2} \varepsilon^{i}, \mathrm{D}^{2} \kappa_{i}^{1}, \mathrm{D}^{2} \kappa_{i}^{2}, \mathrm{D}^{2} \tau_{i}\right)
$$

Finally, $\mathbf{K}_{i}$ is the matrix of tangent moduli which depends on the element energy $W_{i}$ and is defined as

$$
\mathbf{K}_{i}\left(\mathbf{E}_{i}\right)=\left(\begin{array}{ccccc}
W_{i, \varepsilon^{i-1} \varepsilon^{i-1}} & 0 & W_{i, \kappa_{i}^{1} \varepsilon^{i-1}} & W_{i, \kappa_{i}^{2} \varepsilon^{i-1}} & W_{i, \tau_{i} \varepsilon^{i-1}} \\
& W_{i, \varepsilon^{i} \varepsilon^{i}} & W_{i, \kappa_{i}^{1} \delta \varepsilon^{i}} & W_{i, \kappa_{i}^{2} \varepsilon^{i}} & W_{i, \tau_{i} \varepsilon^{i}} \\
& & W_{i, \kappa_{i}^{1} \kappa_{i}^{1}} & W_{i, \kappa_{i}^{1} \kappa_{i}^{2}} & W_{i, \kappa_{i}^{1} \tau_{i}} \\
& \operatorname{sym} . & & W_{i, \kappa_{i}^{2} \kappa_{i}^{2}} & W_{i, \kappa_{i}^{2} \tau_{i}} \\
& & & & W_{i, \tau_{i} \tau_{i}}
\end{array}\right),
$$

where a subscript commas denote partial derivatives, e.g., $W_{i, \tau_{i} \tau_{i}}=\frac{\partial^{2} W_{i}}{\partial \tau_{i}^{2}}$. Explicit expressions for $\boldsymbol{\Sigma}_{i}$ and $\mathbf{K}_{i}$ for the potential energy (6.2) are provided in Appendix D.

The terms in the right-hand side of (7.11) are known as elastic stiffness and geometric stiffness, respectively. If the external load $\mathbf{F}_{\text {ext }}$ is a follower load, there is an additional contribution to the stiffness coming from the gradient of the force, as shown in one of the examples in Section 8.1.

\subsection{Inelastic case}

In the case of a beam made of a viscous or visco-elastic material, $W_{i}\left(\mathbf{E}_{i}, \mathbf{z}_{i}\right)$ is the potential energy and $\mathcal{D}_{i}\left(\dot{\mathbf{E}}_{i}, \dot{\mathbf{z}}_{i}\right)$ the dissipation potential of the element $i, i \in\{1, \cdots, n-2\}$. Inspired by the variational constitutive updates introduced by Ortiz and Stainier (1999) we define $\mathbf{z}_{i}^{\dagger}\left(\mathbf{E}_{i}\right)$ as the solution of the following equation

$$
\frac{\partial W_{i}}{\partial \mathbf{z}_{i}}\left(\mathbf{E}_{i}, \mathbf{z}_{i}\right)+\frac{\partial \mathcal{D}_{i}}{\partial \dot{\mathbf{z}}_{i}}\left(\frac{\mathbf{E}_{i}-\left(\mathbf{E}_{i}\right)_{\star}}{\Delta t}, \frac{\mathbf{z}_{i}-\left(\mathbf{z}_{i}\right)_{\star}}{\Delta t}\right)=0 .
$$

As the quantities $\left(\mathbf{E}_{i}\right)_{\star}$ and $\mathbf{z}_{i}^{\dagger}\left(\mathbf{E}_{i}\right)$ vary from one time step to next, $\mathbf{z}_{i}^{\dagger}\left(\mathbf{E}_{i}\right)$ depends on time $t_{\star}$, even though this is implicit in our notation. The relation above ensures the equality between viscous and elastic stresses, with a formalism akin to that of, e.g., Biot (1954); Halphen and Nguyen (1975).

Following Ortiz and Stainier (1999), we introduce the condensed potentials by inserting the solution $\mathbf{z}_{i}^{\dagger}\left(\mathbf{E}_{i}\right)$ of the above equation into the original potentials,

$$
W_{i}^{\dagger}\left(\mathbf{E}_{i}\right)=W_{i}\left(\mathbf{E}_{i}, \mathbf{z}_{i}^{\dagger}\left(\mathbf{E}_{i}\right)\right), \quad \mathcal{D}_{i}^{\dagger}\left(\mathbf{E}_{i}\right)=\mathcal{D}_{i}\left(\frac{\mathbf{E}_{i}-\left(\mathbf{E}_{i}\right)_{\star}}{\Delta t}, \frac{\mathbf{z}_{i}^{\dagger}\left(\mathbf{E}_{i}\right)-\left(\mathbf{z}_{i}\right)_{\star}}{\Delta t}\right) .
$$

The discrete elastic and viscous stress then take the form, respectively,

$$
\begin{aligned}
& \mathbf{\Sigma}_{i}^{\dagger}\left(\mathbf{E}_{i}\right)=\left(W_{i, \varepsilon^{i-1}}^{\dagger}, W_{i, \varepsilon^{i}}^{\dagger}, W_{i, \kappa_{i}^{1}}^{\dagger}, W_{i, \kappa_{i}^{2}}^{\dagger}, W_{i, \tau_{i}}^{\dagger}\right), \\
& \mathbf{N}_{i}^{\dagger}\left(\mathbf{E}_{i}\right)=\left(\mathcal{D}_{i, \varepsilon^{i-1}}^{\dagger}, \mathcal{D}_{i, \varepsilon^{i}}^{\dagger}, \mathcal{D}_{i, \kappa_{i}^{1}}^{\dagger}, \mathcal{D}_{i, \kappa_{i}^{2}}^{\dagger}, \mathcal{D}_{i, \tau_{i}}^{\dagger}\right) .
\end{aligned}
$$

Note that the derivatives with respect to the components of $\mathbf{E}_{i}$ in (7.15) and (7.15) involve a dependence on $\mathbf{z}^{\dagger}\left(\mathbf{E}_{i}\right)$. For example,

$$
\frac{\partial W_{i}^{\dagger}}{\partial \varepsilon^{i}}=\left[\frac{\partial W_{i}}{\partial \varepsilon_{i}}\right]_{\mathbf{z}_{i}=\mathbf{z}_{i}^{\dagger}}+\left[\frac{\partial W_{i}}{\partial \mathbf{z}_{i}}\right]_{\mathbf{z}_{i}=\mathbf{z}_{i}^{\dagger}} \cdot\left(\frac{\partial \mathbf{z}_{i}^{\dagger}}{\partial \varepsilon^{i}}\right)
$$




$$
\frac{\partial \mathcal{D}_{i}^{\dagger}}{\partial \varepsilon^{i}}=\left[\frac{\partial \mathcal{D}_{i}}{\partial \varepsilon_{i}}\right]_{\mathbf{z}_{i}=\mathbf{z}_{i}^{\dagger}}+\frac{1}{\Delta t}\left[\frac{\partial \mathcal{D}_{i}}{\partial \dot{\mathbf{z}}_{i}}\right]_{\mathbf{z}_{i}=\mathbf{z}_{i}^{\dagger}} \cdot\left(\frac{\partial \mathbf{z}_{i}^{\dagger}}{\partial \varepsilon^{i}}\right) .
$$

Note that the particular linear combination of $\boldsymbol{\Sigma}_{i}^{\dagger}\left(\mathbf{E}_{i}\right)$ and $\mathbf{N}_{i}^{\dagger}\left(\mathbf{E}_{i}\right)$ that enters into (7.16), namely $\boldsymbol{\Sigma}_{i}^{\dagger}+\Delta t \mathbf{N}_{i}^{\dagger}$, makes the last terms in each of the above equations cancel with one another due to (7.14). This cancellation, however, does not take place in the calculation of the matrix of tangent moduli. For the constitutive relation (6.7) considered here, (7.14) is a simple algebraic equation and the partial derivatives $\frac{\partial \mathbf{z}_{i}^{\dagger}}{\partial \varepsilon^{i-1}}, \frac{\partial \mathbf{z}_{i}^{\dagger}}{\partial \varepsilon^{i}}$, $\frac{\partial \mathbf{z}_{i}^{\dagger}}{\partial \kappa_{i}^{\dagger}}, \frac{\partial \mathbf{z}_{i}^{\dagger}}{\partial \kappa_{i}^{2}}$ and $\frac{\partial \mathbf{z}_{i}^{\dagger}}{\partial \tau_{i}}$ are available in closed form, as shown in Appendix D.

With this definition of the elastic and viscous internal forces, the principle of virtual work leads to a set of nonlinear, coupled equations for the components of the generalized acceleration $\mathbf{a}_{\Delta t}$ : upon projection onto the basis $\left(\tilde{\mathbf{u}}^{k}\right)_{k \in\{1, \cdots, 4 n-1\}}$, it writes

$$
\sum_{i=1}^{n-2}\left[\mathbf{a}_{\Delta t} \cdot \mathbf{C}_{i}^{T} \cdot \mathbf{M}_{i}\right] \cdot\left(\mathbf{C}_{i} \cdot \tilde{\mathbf{u}}^{k}\right)+\sum_{i=1}^{n-2}\left[\left(\boldsymbol{\Sigma}_{i}^{\dagger}+\Delta t \mathbf{N}_{i}^{\dagger}\right) \cdot \mathrm{D} \mathbf{E}_{i}\right] \cdot\left(\mathbf{C}_{i} \cdot \tilde{\mathbf{u}}^{k}\right)-F_{\mathrm{ext}}^{k}=0,
$$

where $\mathbf{a}_{\Delta t}$ is discretized in time using an appropriate finite-difference scheme (i.e. we use $\mathbf{a}_{\Delta t}=\mathcal{G}\left(\mathbf{u}, \mathbf{u}_{\star}, \mathbf{v}_{\star}, \mathbf{a}_{\star}\right)$ from (7.2)). This equation is analogous to the evolution equation in the conservative case (7.9), except for the additional viscous contribution $\mathbf{N}_{i}^{\dagger}$ to the internal stress, and for the additional dependence of the elastic stress on the internal variable $\mathbf{z}_{i}$ reflected by the function $\boldsymbol{\Sigma}_{i}^{\dagger} \neq \boldsymbol{\Sigma}_{i}$. We denote by $\mathbf{F}_{\text {int }}^{\dagger}$ the resultant of internal stress appearing in the equation (7.16): restoring the implicit arguments and denoting $\mathbf{S}_{i}=\boldsymbol{\Sigma}_{i}^{\dagger}+\Delta t \mathbf{N}_{i}^{\dagger}$

$$
\mathbf{F}_{\text {int }}^{\dagger}(\mathbf{u})=\sum_{i=1}^{n-2} \mathbf{S}_{i}\left(\mathbf{E}_{i}\left(\mathbf{C}_{i} \cdot \mathbf{u}\right)\right) \cdot \mathrm{D} \mathbf{E}_{i}\left(\mathbf{C}_{i} \cdot \mathbf{u}\right) \cdot \mathbf{C}_{i}
$$

The stiffness matrix is given by equation (7.11) for the elastic case, but with $\boldsymbol{\Sigma}_{i}$ replaced with $\mathbf{S}_{i}$, it is replaced by

$$
\mathbf{K}_{i}^{\dagger}=\left(\begin{array}{ccccc}
\mathbf{S}_{i, \varepsilon^{i-1} \varepsilon^{i-1}} & 0 & \mathbf{S}_{i, \kappa_{i}^{1} \varepsilon^{i-1}} & \mathbf{S}_{i, \kappa_{i}^{2} \varepsilon^{i-1}} & \mathbf{S}_{i, \tau_{i} \varepsilon^{i-1}} \\
& \mathbf{S}_{i, \varepsilon^{i} \varepsilon^{i}} & \mathbf{S}_{i, \kappa_{i}^{1} \varepsilon^{i}} & \mathbf{S}_{i, \kappa_{i}^{2} \varepsilon^{i}} & \mathbf{S}_{i, \tau_{i} \varepsilon^{i}} \\
& & \mathbf{S}_{i, \kappa_{i}^{1} \kappa_{i}^{1}} & \mathbf{S}_{i, \kappa_{i}^{2} \kappa_{i}^{1}} & \mathbf{S}_{i, \tau_{i} \kappa_{i}^{1}} \\
& \operatorname{sym} & & \mathbf{S}_{i, \kappa_{i}^{2} \kappa_{i}^{2}} & \mathbf{S}_{i, \tau_{i} \kappa_{i}^{2}} \\
& & & & \\
& & & \mathbf{S}_{i, \tau_{i} \tau_{i}}
\end{array}\right) .
$$

Explicit expressions of $\mathbf{S}_{i}$ and $\mathbf{K}_{i}^{\dagger}$ for the viscous and visco-elastic laws, (6.5) and (6.7), respectively, are provided in Appendix D. Numerical examples involving viscous beams will be presented in Section 8.2.

Algorithm 2 summarizes the iterative solution of a quasistatic load step for a discrete beam using Newton- 
Raphson iteration. In this algorithm, we use the index $i, i \in\{1, \cdots, n-2\}$ to iterate over elements.

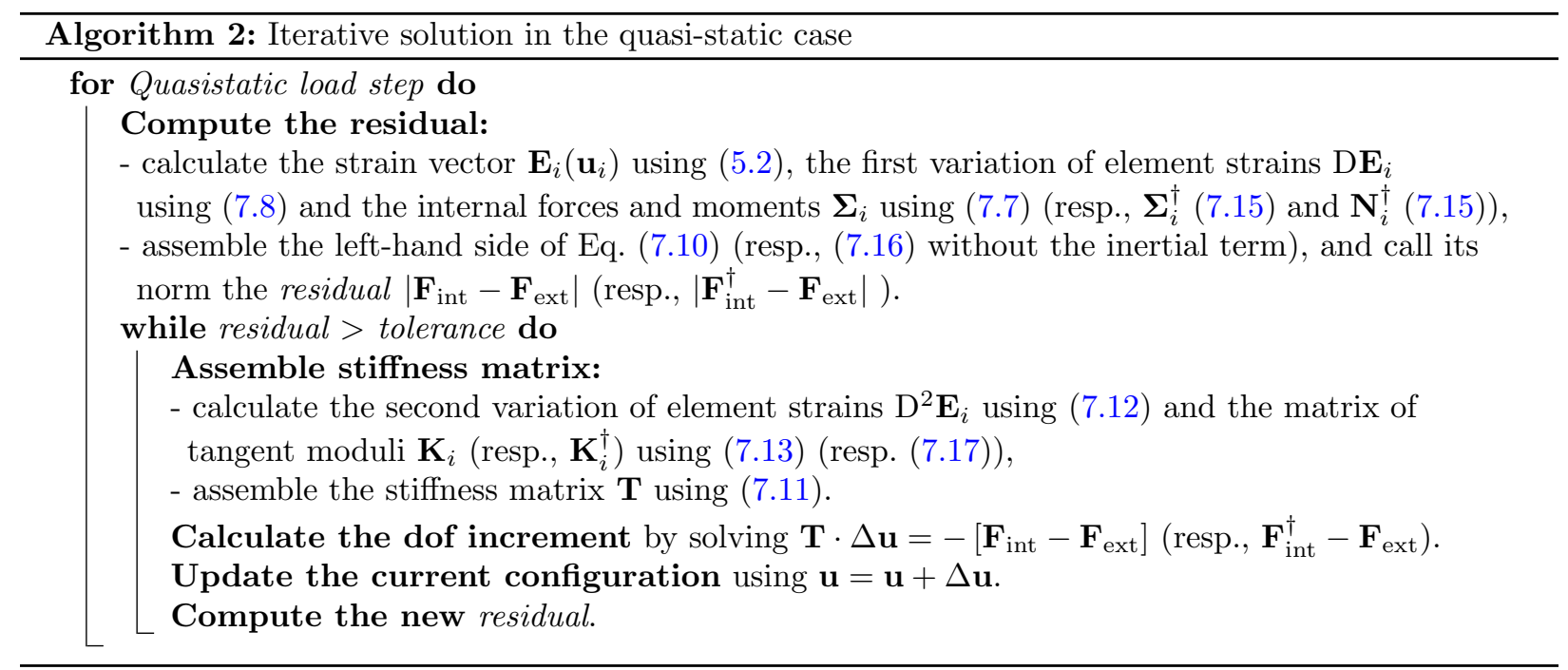

\section{Examples}

Previous work on the discrete beam model was focused on specific material models, namely linear elasticity and linear viscosity. The versatile framework presented here is validated for various types of constitutive behavior. The goal is to verify the accuracy of our numerical scheme and demonstrate its ability to solve a variety of initial value and boundary value problems: our benchmark examples include three quasistatic problems for inextensible elastic beams described by the constitutive laws (6.1) in 2D and in 3D (Section 8.1), two 2D quasistatic problems for viscous beams made of a Newtonian fluid described by (6.3) (Section 8.2), and a dynamic problem involving a visco-elastic beam described by (6.7) (Section 8.3).

\subsection{Quasistatic problems involving elastic beams}

We start with weakly extensible, linearly elastic beams, also known as Elasticas. The material behavior is described by the discrete version of the quadratic elastic potential (6.2). We first study the pinching of a circular, planar elastica, then the bending of a $2 \mathrm{D}$ beam subject to an end moment, and finally the equilibrium of a curly hair under gravity. For each of these three examples, we compare the solution of the discrete beam model to numerical solutions of the 1D boundary value equilibrium problem using a shooting method and standard integration techniques for ordinary differential equations. We consider beams made of an incompressible material in $3 \mathrm{D}$, so that $\mu=E / 3$ in (6.2). Entire solution branches of the discrete model (7.9) are obtained using a path-following method: the value of the loading parameter is progressively increased and the equilibrium is obtained at each load step.

Elastica in 2D. We consider a planar Elastica rolled into a circular arch of initial radius $R=0.4933$ (which corresponds to an arc length $L \approx 3$ ), subject to two diametrically opposite pinching forces of magnitude $F$, as illustrated in Figure 8.1(a). The bending modulus in $(6.2)$ is set to $E I_{1} \approx 0.74$, the magnitude of the final load $F$ is adjusted so as to have $\frac{L^{2} F}{2 E I_{1}}=4.87(F=6)$ and we set the slenderness parameter $I_{1} / A L^{2} \approx 10^{-9}$.

We focus on solutions retaining the symmetries of the data, and solve the problem on a quarter of the arch as sketched in Figure 8.1(b): the boundary conditions relevant to the assumed symmetry must be enforced on the endpoints of the quarter circle, i.e., the rotation must be blocked there. To achieve this, we surround the endpoints of the quarter circle with two equally spaced vertices, see the arrows in Figure 8.1(b), and constraint the displacements of each of these pairs of vertices in such a way that the rotation of the corresponding (terminal) segment is zero. In the continuum limit, the rotation is effectively blocked on the midpoints of the terminal segments, which by construction match the endpoints of the physical domain (quarter circle), hence a good accuracy. 


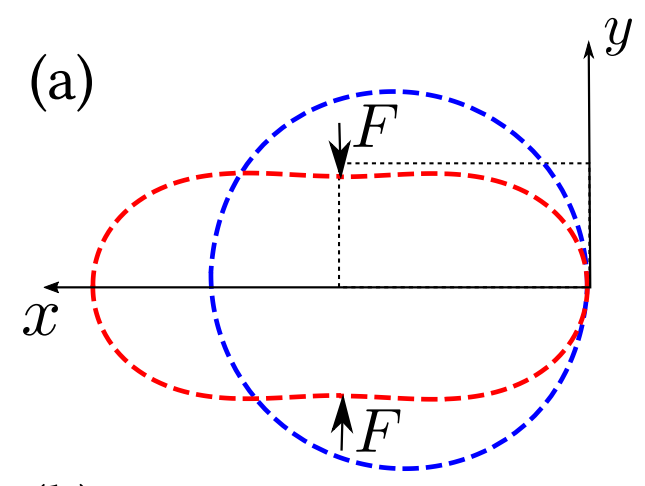

(b)
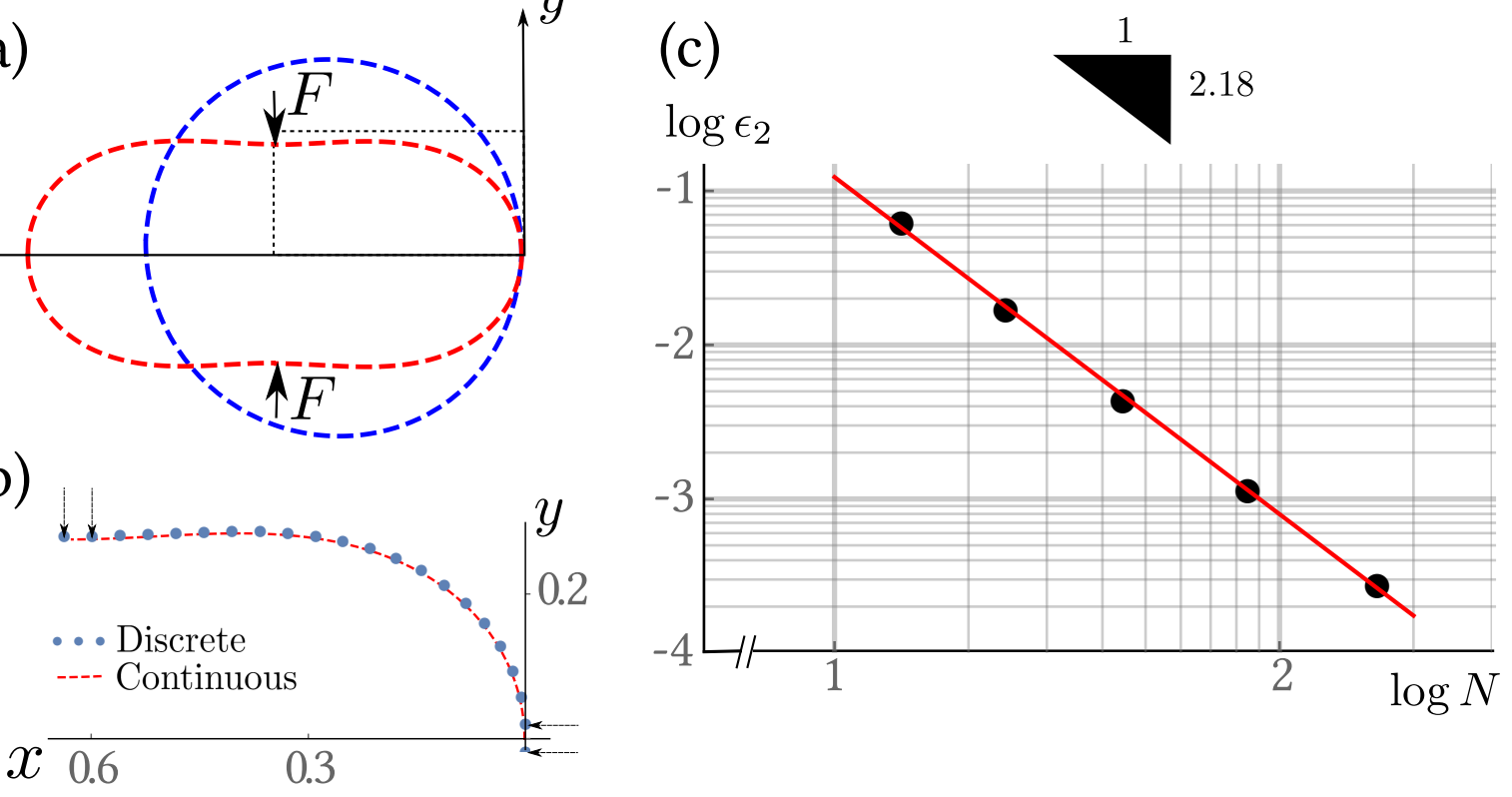

Figure 8.1: Validation for a pinched Elastica in 2D. (a) Undeformed (blue) and deformed (red) configurations of a circular Elastica (with initial radius $R=0.4933$ ) subject to a pinching force $F$ (with $\frac{L^{2} F}{2 E I_{1}}=4.87$ ). (b) Comparison between results of the discrete model with $n=22$ (blue dots) and a numerical solution obtained by the shooting method applied to the ordinary differential equations for the equilibrium (dashed red line). (c) Convergence of the error (using the relative discrete $L^{2}$-norm) as a function of the number of degrees of freedom $N=2 n$ (dots) drawn in a log-log plot, shown with its logarithmic regression (red line) and the measured slope.

The discrete numerical solution calculated for $n=22(N=44$ degrees of freedom $)$ is depicted by the blue dots in Figure 8.1(b). It is in excellent agreement with the solution obtained by solving the continous boundary value equilibrium problem using a shooting method (as shown by the dashed red line). We further evaluate the convergence of our discrete solution by computing the relative norm of the error defined as

$$
\epsilon_{2}=\frac{\sum_{k=0}^{n}\left[\left(x\left(s_{k}\right)-\tilde{x}\left(s_{k}\right)\right)^{2}+\left(y\left(s_{k}\right)-\tilde{y}\left(s_{k}\right)\right)^{2}\right]}{\left.\sum_{k=0}^{n}\left[x\left(s_{k}\right)^{2}+y\left(s_{k}\right)\right)^{2}\right]}
$$

where $\left\{s_{1}, \cdots, s_{n}\right\}$ denote the curvilinear coordinates of the vertices, $(x, y)$ denotes the reference solution obtained by the shooting method and $(\tilde{x}, \tilde{y})$ denotes the solution of the discrete model. As may be expected from a beam model (requiring square-integrable second derivatives and hence leading to FEM error estimates that scale quadratically with element size), we achieve quadratic convergence, as demonstrated in Figure 8.1(c).

The second example demonstrates applied point-like moments. We consider a naturally straight, initially horizontal beam in 2D, having normalized length $L=1$, clamped at one end and subjected to a bending moment of mangitude $M$ at its free end, as depicted in Figure 8.2(a). The bending modulus is set to $E I_{1}=0.74$, the final value of the loading moment is adjusted so as to have $\frac{M L}{E I_{1}}=3.1(M=2.3)$ and we set the slenderness parameter $I_{1} / A L^{2} \approx 10^{-9}$. As in the previous example, we apply a clamped boundary condition at $s=0$ by defining an additional vertex at $\mathbf{x}_{0}$ outside to the left of the domain $[0, L]$ (not shown in the figure) and blocking the displacements of $\mathbf{x}_{0}$ and $\mathbf{x}_{1}$. We apply the external moment by adding one extra vertex outside to the right of the domain $[0, L]$ and by setting up two forces perpendicular to the end segment $\mathbf{t}^{n-2}=\mathbf{d}_{3}^{n-2}$ (e.g. aligned with $\mathbf{d}_{1}^{n-2}$ ) and of opposite magnitudes $\mathbf{F}_{ \pm}= \pm\left(M / l_{0}^{n-2}\right) \mathbf{d}_{1}^{n-2}$ on the last two vertices at $\mathbf{x}_{n-1}$ and $\mathbf{x}_{n-2}$, respectively. Note that these forces contribute to the stiffness matrix for they involve a non-zero variation: for a small increment in displacement $\delta \mathbf{u}, \delta \mathbf{F}_{ \pm}= \pm\left(M / l_{0}^{n-2}\right) \operatorname{Dd}_{1}^{n-2} \cdot \mathbf{C}_{n-2} \cdot \delta \mathbf{u}$ where $\mathrm{Dd}_{1}^{n-2}$ is given by (5.7). As for the pinched elastica, we compare our results to a solution obtained by 

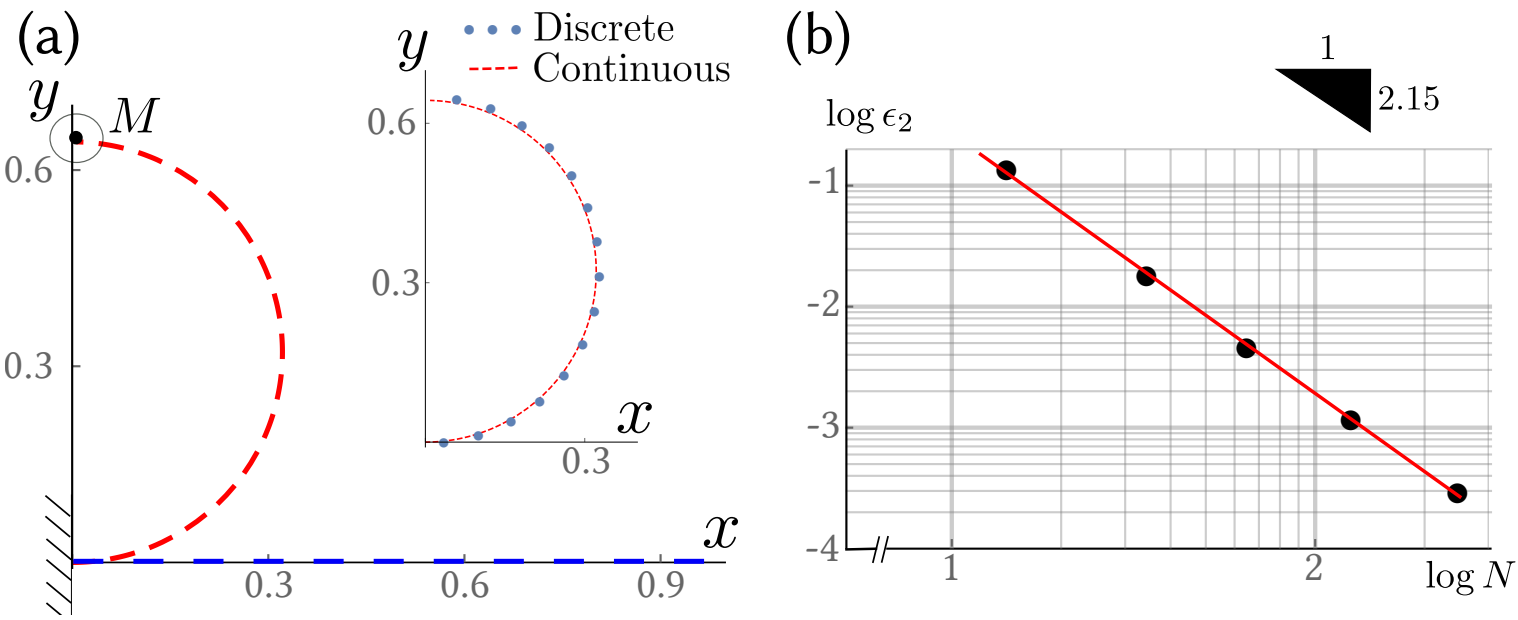

Figure 8.2: Elastica bent under an end moment. (a) Undeformed (blue) and deformed (red) configurations of an initially straight Elastica (of length $L=1$ ) subject to an end moment $M$ (with $\frac{M L}{E I_{1}}=3.1$ ). Inset: comparison between results obtained with the discrete model with $n=16$ (blue dots) and a direct numerical integration of the continuous equations (dashed red line). (b) Convergence of the error (in the relative discrete $L^{2}$-norm) as a function of the number of degrees of freedom $N=2 n$ (dots), drawn in a log-log plot and shown with its logarithmic regression (red line).

a direct numerical integration of the continuous equilibrium equations, as shown in Figure 8.2(a) for $n=22$. The quadratic convergence of the error is confirmed in Figure 8.2(b).

Elastica in 3D: suspended curly hair under gravity. With the aim to validate the model in $3 \mathrm{D}$, we determine the equilibrium shape of a suspended curly hair of lineic weight $w$ and length $L$. Natural curvature and twist $\tau_{0}$ are accounted for by modifying the elastic energy (6.2) to

$W_{i}=\frac{1}{2}\left(\frac{E A}{2}\left(l_{0}^{i-1}\left(\varepsilon^{i-1}\right)^{2}+l_{0}^{i}\left(\varepsilon^{i}\right)^{2}\right)+\left(\tilde{l}_{i}\right)_{0} E I_{1}\left(\tilde{\kappa}_{i}^{1}-\left(\tilde{\kappa}_{0}^{1}\right)_{i}\right)^{2}+\left(\tilde{l}_{i}\right)_{0} E I_{2}\left(\tilde{\kappa}_{i}^{2}-\left(\tilde{\kappa}_{0}^{2}\right)_{i}\right)^{2}+\left(\tilde{l}_{i}\right)_{0} \mu J\left(\tilde{\tau}_{i}-\left(\tilde{\tau}_{0}\right)_{i}\right)^{2}\right)$

where $\left(\tilde{\kappa}_{0}^{I}\right)_{i}=\kappa_{0}^{I} /\left(\tilde{l}_{i}\right)_{0}$ for $I \in\{1,2\},\left(\tilde{\tau}_{0}\right)_{i}=\tau_{0} /\left(\tilde{l}_{i}\right)_{0}$, and $\kappa_{0}^{1}, \kappa_{0}^{2}$ denote the natural curvatures along the material directors $\mathbf{d}_{1}$ and $\mathbf{d}_{2}$, respectively, and $\tau_{0}$ denotes the natural twist (see for example Basset (1895)). In this example we set $\kappa_{0}^{1}=\kappa_{\text {nat }}, \kappa_{0}^{2}=0$ and $\tau_{0}=0$.

Under the assumption $I_{1}=I_{2}$ relevant to circular cross-sections, the hair's shape is controlled by two parameters (Audoly and Pomeau, 2010): the dimensionless natural curvature $\bar{\kappa}=\kappa_{\text {nat }} L$ and the dimensionless lineic weight $\bar{w}=w / \kappa_{\text {nat }}^{3} E I_{1}$. The equilibrium problem admits a planar solution for small natural curvatures, which bifurcates into a 3D configuration comprising twist as $\kappa_{\text {nat }}$ increases (Miller et al., 2014). This behavior is reproduced by the discrete beam model as follows.

We use the same set of dimensionless parameters as in Miller et al. (2014), and set the torsion modulus to $\mu J=0.5$, the lineic weight to $w=2.8 \cdot 10^{-6}$ and the slenderness parameter to $I_{1} / A L^{2} \approx 10^{-5}$. We follow the branch of nonlinear solutions by progressively increasing the natural curvature until we reach $(\bar{\kappa}, \bar{w})=(8.4,108)$. The hair is naturally straight with a small natural twist $\tau_{0}=10^{-5}$, and we apply a clamping condition $\left(\mathbf{d}_{1}, \mathbf{d}_{2}, \mathbf{d}_{3}\right)_{s=0}=\left(\mathbf{e}_{y}, \cos \theta_{0} \mathbf{e}_{x}-\sin \theta_{0} \mathbf{e}_{z},-\cos \theta_{0} \mathbf{e}_{z}-\sin \theta_{0} \mathbf{e}_{x}\right)$ with $\theta_{0}=\frac{\pi}{20}$, as shown in Figure 8.3(a). The small parameters $\tau_{0}$ and $\theta_{0}$ help trigger the out-of-plane buckling.

When we progressively increase the natural curvature, the hair first bends, staying close to the $\left(\mathbf{e}_{x}, \mathbf{e}_{z}\right)$ plane and later adopts a 3D shape, as shown in Figure 8.3(a). We compare our discrete, numerical solution with one obtained by a numerical solution of the continuous equations of equilibrium using the continuation software AUTO (Doedel et al., 2007). There is good agreement between the two solutions, as shown in Figure 8.3(a) for $n=121$. The convergence is quadratic, as for the $2 \mathrm{D}$ examples, see Figure 8.3(b). This examples completes our validation of elasticity problems. 

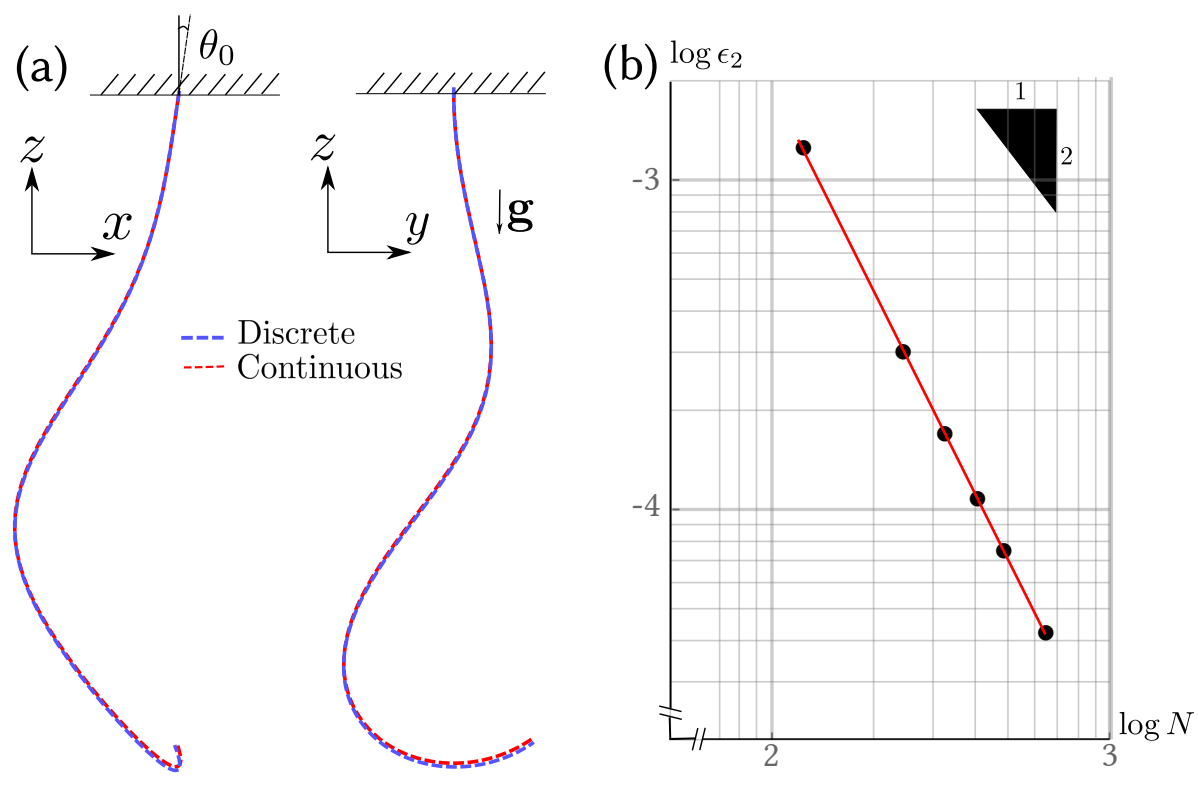

Figure 8.3: Curly hair under gravity. Beam having natural curvature $\kappa_{\text {nat }} L=8.4$, an isotropic bending modulus $E I=1$, a torsion modulus $\mu J=0.5$ and a lineic weight $w$, with $\frac{w}{E I \kappa_{\text {nat }}^{3}}=108$. (a) Comparison between results obtained with the discrete model with $n=121$ (dashed blue line) and by integrating continuous equilibrium equations (dashed red line). (b) Convergence of the error (in the relative discrete $L^{2}$-norm) as a function of the number of degrees of freedom $N=4 n-1$ (dots) drawn in log-log scale, shown together with its logarithmic regression (red line).

\subsection{Quasistatic evolution of viscous beams}

We proceed to illustrate viscous material behavior, as defined by the dissipation potential (6.5). We solve numerically the evolution equation of the discrete problem in Eq. (7.16), neglecting inertia. We focus on two 2D examples: the stretching of a vertical thread of viscous fluid under its own weight, and the sagging of a horizontal viscous thread under the combined effects of gravity and surface tension.

Stretching of a vertical filament under its own weight. We consider an initially straight, vertical viscous filament of homogeneous mass density $\rho=100$ and viscosity $\eta=2$ subject to gravity, as pictured in Figure 8.4(a). For this simple example, we neglect the effects of surface tension $(\gamma=0)$, so that we can derive a reference analytical solution. We denote by $\lambda(s)=1+\varepsilon(s)$ the axial stretch. The evolution is governed by the balance of gravity and viscous forces,

$$
\rho g(L-s)-3 \eta \frac{\dot{\lambda}}{\lambda^{2}}=0
$$

which can be integrated with respect to time $t$, yielding $\lambda=1 /(1-\rho g(L-s) t / 3 \eta)$. The result at short times $t \ll 1$ can be re-written as $\lambda \approx 1+\rho g(L-s) t / 3 \eta$. Integrating this last expression yields the axial displacement $\xi_{z}(s, t)=\left[\boldsymbol{r}(s, t)-\boldsymbol{r}_{0}(s, t)\right] \cdot \mathbf{e}_{z}$ at short times as

$$
\xi_{z}(s, t)=\frac{\rho g}{3 \eta}\left(L s-\frac{s^{2}}{2}\right) t .
$$

This solution at $t=10^{-4}$ is compared with discrete numerical results obtained with $n=20$ in Figure 8.4(a) for a viscous filament of initial length $L_{0}=1$. Convergence is evaluated to be linear, as shown in Figure 8.4(b). Note that, with our choice of discretization, the convergence is quadratic when bending and twisting are considered, as seen in Section 8.1 (inextensible beam model), while it is linear for pure stretching as shown here, owing to the differences in the order of derivation in the weak forms for the stretching and 
(a)

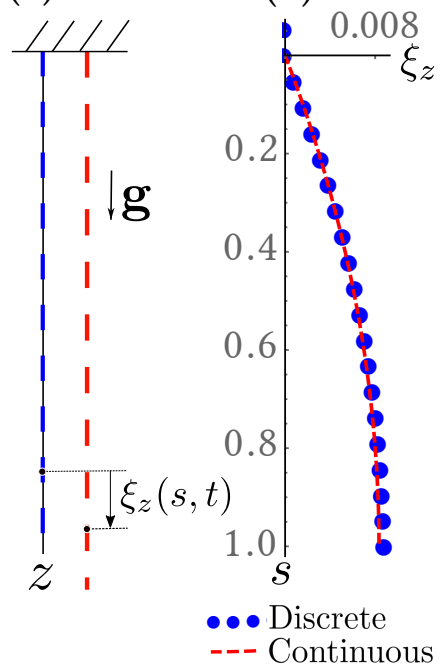

(c)

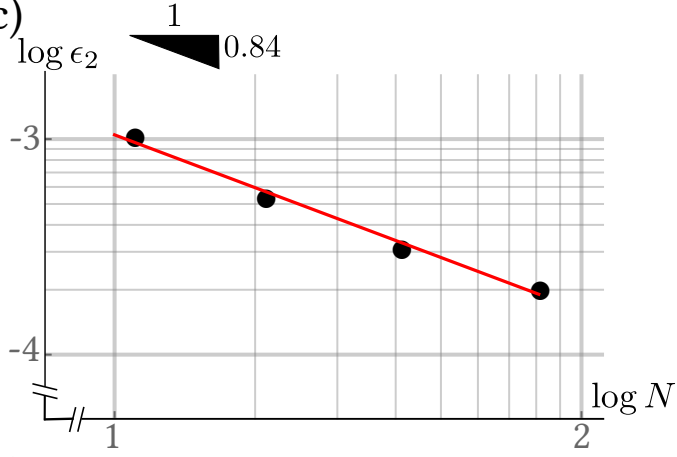

(d) $L(t)$

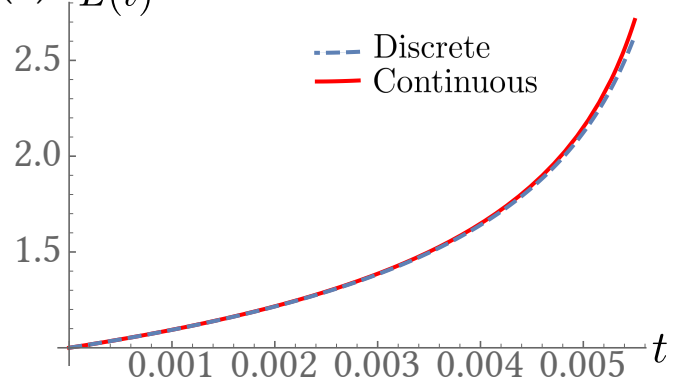

Figure 8.4: Straight viscous beam sagging under gravity. Initially straight thread of viscous fluid having viscosity $\eta=2$, density $\rho=100$, initial radius $r_{0}=10^{-2}$ and length $L_{0}=1$ sagging under gravity. (a) Sketch of the undeformed (blue) and deformed (red) configurations. (b) Comparison of the axial displacement at $t=10^{-4}$ evaluated with the discrete model with $n=20$ (blue dots) and with the analytical prediction (8.2)(dashed red line). (c) Convergence of the error at $t=10^{-4}$, as a function of the number of degrees of freedom $N=n$ (dots), drawn in a log-log plot and shown along with its logarithmic regression (red line). (d) Comparison of the current length $L(t)$ over long times evaluated with the discrete model with $n=20$ (dashed blue line) and with the analytical prediction (8.3) (red line). 
(a)

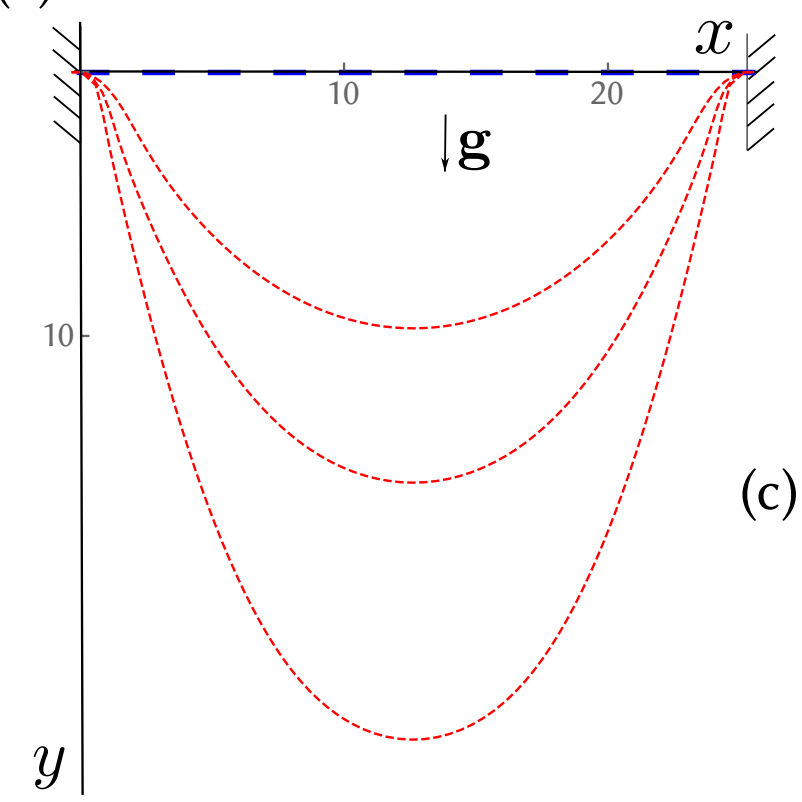

(b)

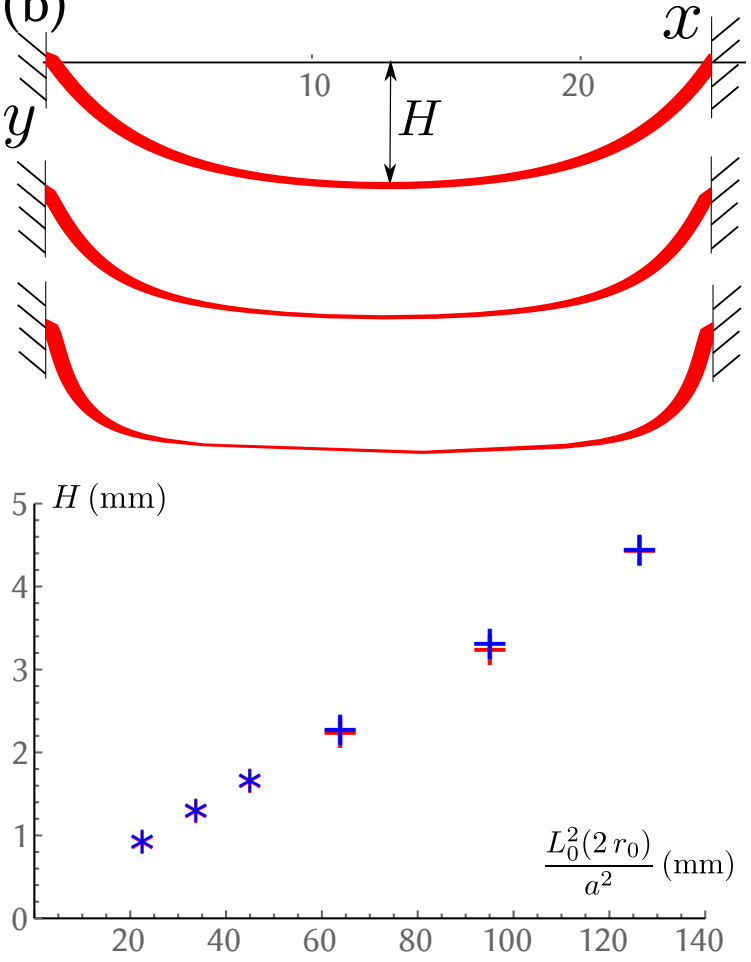

Figure 8.5: A hanging viscous filament sagging under its own weight. (a) Typical catenary shapes, obtained when gravity dominates surface tension. Undeformed configuration (blue) and deformed configurations (centerline is shown with a red dashed line, thickness is not represented) at $t=0.08 \mathrm{~s}, t=0.16 \mathrm{~s}$ and $t=0.24 \mathrm{~s}$ respectively, for $L_{0}=25 \mathrm{~mm}$ and $r_{0}=0.875 \mathrm{~mm}, \eta=10 \mathrm{~Pa} . \mathrm{s}$ and $\gamma=0.02 \mathrm{~kg} . \mathrm{s}^{-2}$. Simulations are run with $n=80$. (b) Typical U-shapes, obtained when surface tension dominates over gravity. Deformed configurations are drawn in red, with a thickness proportional to the thickness predicted by simulations, at $t=0.6 \mathrm{~s}, t=0.8 \mathrm{~s}$ and $t=1 \mathrm{~s}$, respectively, for $L_{0}=25 \mathrm{~mm}, r_{0}=0.2 \mathrm{~mm}, \eta=10 \mathrm{~Pa} . \mathrm{s}$ and $\gamma=0.02 \mathrm{~kg} . \mathrm{s}^{-2}$. Simulations are run with $n=80$. (c) Final height $H$ in the U-shape regime for $\eta \in\{5,10\}$ Pa.s (resp., red and blue symbols) and $L_{0} \in\{15,25\} \mathrm{mm}$ (resp., stars and crosses) at $t=0.21 \mathrm{~s}$, obtained from simulations run with $n=20$.

bending problems, as in classical bar and beam models. Finally, we compare the evolution of the current length of the filament $L(t)$, which is given by the exact formula

$$
L(t)=\int_{0}^{L_{0}} \lambda(s, t) \mathrm{d} s=\int_{0}^{L_{0}} \frac{1}{1-\frac{\rho g(L-s) t}{3 \eta}} \mathrm{d} s .
$$

The evolution of $L(t)$ for $t<3 \eta / \rho g L_{0} \approx 0.006$ (value for which $\lambda(s, t)$ blows) calculated with the numerical model is plotted in Figure 8.4(d) and shows good agreement with the exact prediction (8.3).

Competition between viscous forces and surface tension: the viscous catenary. We study the shape of an initially horizontal filament clamped at both endpoints under its own weight, as in the experiments of Le Merrer et al. (2008). In all our numerical examples, parameters are expressed in default units. Here we use specific units reflecting the units of this experimental study, where silicone oils are used. The beam is initially homogeneous with initial radius in the range $r_{0} \in[0.1,0.875] \mathrm{mm}$, initial length $L_{0}$ in the range $L_{0} \in[15,25] \mathrm{mm}$ and density $\rho=1 \mathrm{~g} \cdot \mathrm{cm}^{-3}$. Two values of the viscosity are considered, $\eta \in\{5,10\} \mathrm{Pa} \cdot \mathrm{s}$. A surface tension $\gamma=0.02 \mathrm{~kg} \cdot \mathrm{s}^{-2}$ is included, the two ends of the thread are clamped at their initial position and the time step is set to $\Delta t=1 \mathrm{~ms}$.

In the simulations, the thread progressively sags under the effect of gravity, as illustrated in Figure 8.5(a). This behavior is similar to what is observed in the experiments. Since the initial radius distribution as a function of the arc length is non-uniform and ill-controlled in the experiments of Le Merrer et al. (2008), 
we can hope for a qualitative, but not a quantitative agreement between simulations and experiments. The experiments have revealed two regimes, referred to as the 'catenary' and 'U-shape' regimes: those regimes are recovered in the simulations, see the parts (a) versus (b) of the figure. The thickness of the filament remains homogeneous in the catenary regime (filament thickness is not shown, for the sake of legibility) while it is much smaller at the center of the thread than at the endpoints in the U-shape regime, as shown in Figure 8.5(b) and reported in Le Merrer et al. (2008). In Figure 8.5(c), we also reproduce the experimental scaling law for the maximum sag $H$ measured in the U-shape regime: $H \sim \frac{L_{0}^{2}\left(2 r_{0}\right)}{a^{2}}$ where $a^{2}=\frac{\gamma}{\rho g}$, for different values of viscosity, initial length and initial radius.

\subsection{Dynamic problems}

Free vibrations of a cantilever beam. We study the free transverse vibrations of a planar (2D) inextensible cantilever beam of length $L$, bending modulus $E I$, cross-sectional area $A$, and density $\rho$. The beam is horizontal and initially aligned with the axis $\mathbf{e}_{x}$; its weight is neglected. We recall that, under the approximation of small displacement, the angular velocities $\lambda$ of the modes of vibrations of the beam can be found as the eigenvalues of the linearized equations of motion

$$
\frac{E I}{\rho A L^{4}} \bar{f}^{\prime \prime \prime \prime}(\bar{x})-\lambda^{2} \bar{f}(\bar{x})=0,
$$

where $f(x)$ is amplitude of the transverse displacement $\xi_{y}(x, t)=\boldsymbol{r}(x, t) \cdot \mathbf{e}_{y}=\sin (\lambda t) f(x)$ and $\bar{f}(\bar{x})=f(x)$ for $\bar{x}=\frac{x}{L}$ and $\bar{x} \in[0,1]$. The clamped-free boundary conditions write

$$
\bar{f}(0)=0, \quad \bar{f}^{\prime}(0)=0, \quad \bar{f}^{\prime \prime}(1)=0, \quad \text { and } \quad \bar{f}^{\prime \prime \prime}(1)=0 .
$$

The angular eigenfrequencies associated with the first three eigenmodes are found to be $\lambda_{1} \approx 0.69623$, $\lambda_{2} \approx 4.36321$ and $\lambda_{3} \approx 12.2171$, respectively. We use this solution as a mean to verify our numerical method. In the simulation, we set $\frac{E I}{\rho A L^{4}}=0.039$ and perform a numerical eigenvalue analysis of Eq. (7.9) in the absence of any external forces. Degrees of freedom other than the translations in the $\mathbf{e}_{y}$ direction are constrained to zero, which has the effect of removing the torsional and extensional modes. Figure 8.6(a) shows the shapes of the first three eigenmodes obtained from the discrete beam model for $n=20$, together with the exact solution of Eqs. (8.4a) and (8.4b). Figure 8.6(b) demonstrates the convergence of the first three eigenvalues $\lambda$ predicted by the discrete beam model, towards the exact values for the continuous model. As expected, the results obtained from the consistent and lumped mass matrices, respectively, over- and underpredict the eigenvalues.

Visco-elastic snap-through. In this last validation example, we simulate the dynamic snap-through of a planar visco-elastic beam whose behavior is described by (6.7). This problem is analyzed numerically by Gomez et al. (2019). The beam is of length $L$ in its natural, straight, configuration. It is clamped at its two ends. To arrive at an initial pre-deformed, buckled configuration, we rotate the left clamp by an angle $\alpha$, while setting the end-to-end distance to $L-\Delta L$, resulting in the buckled shape shown by the blue line in Figure 8.7(a). We let the beam relax in this initial equilibrium, characterized by the dimensionless parameter $\mu=\alpha(L / \Delta L)^{1 / 2}$. Next, we rigidly move the midpoint of the beam vertically down to the vertical coordinate $w(1 / 2)=-0.38 \sqrt{L \Delta L}$ and let the beam relax over a variable time interval $t_{\text {ind. At a time }}$ denoted as $t=0$, the beam is then released, i.e., the constraint on $w(1 / 2)$ is removed, and the subsequent motion of the beam is simulated solving Eq. (7.16) using a Newmark scheme.

Gomez et al. (2019) showed that, in the limit of a large Deborah number De $=\eta / E_{2} \sqrt{\rho h L^{4} /\left(E_{1} I_{1}\right)}$ (where $I_{1}=h^{3} / 12$ and $h$ is the beam's thickness), the system snaps back to the initial configuration shown in blue in the figure. The snap-through occurs either over a short elastic time scale estimated as $t_{\mathrm{el}}=L^{3} / h \sqrt{E_{1} / \rho} \approx 0.1$ (Gomez et al., 2017), or after a series of oscillations. The second case is referred to by Gomez et al. (2019) as the pseudo-bistable regime. For De $=10$ and $\beta=E_{2} /\left(E_{2}+E_{1}\right)=0.1$, they showed that pseudo-bistability can occur for a given range of the parameter $\mu$.

The numerical parameters are chosen such that the value of the dimensionless number $\mu=1.84$ lies within this range. We run dynamic simulations with $n=25$ vertices, $\Delta t=10^{-4} s$, and no numerical damping in 
(a)

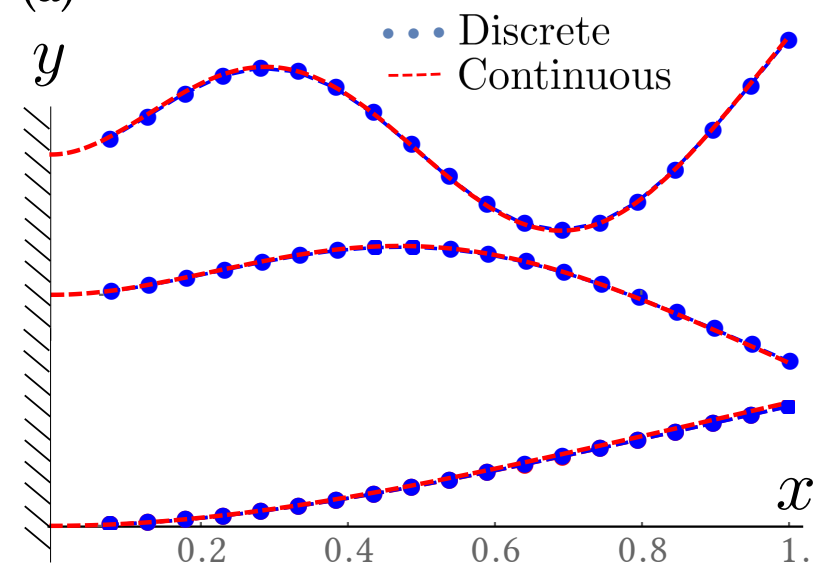

(b)

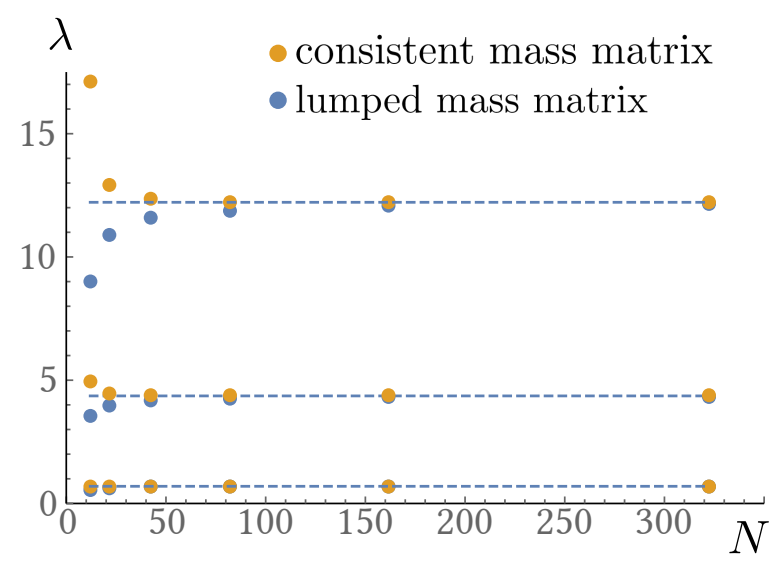

Figure 8.6: Free transverse vibrations of a clamped cantilever beam. (a) Three lowest normalized eigenmodes (with increasing eigenfrequencies from bottom to top) calculated with our discrete model with $n=20$ (blue dots), compared to the reference solution (red line). (b) First angular eigenfrequencies obtained with the lumped (blue) and consistent mass matrix (yellow), compared to the exact prediction for the continuous case (dashed line) as a function of the number of degrees of freedom $N=2 n$.

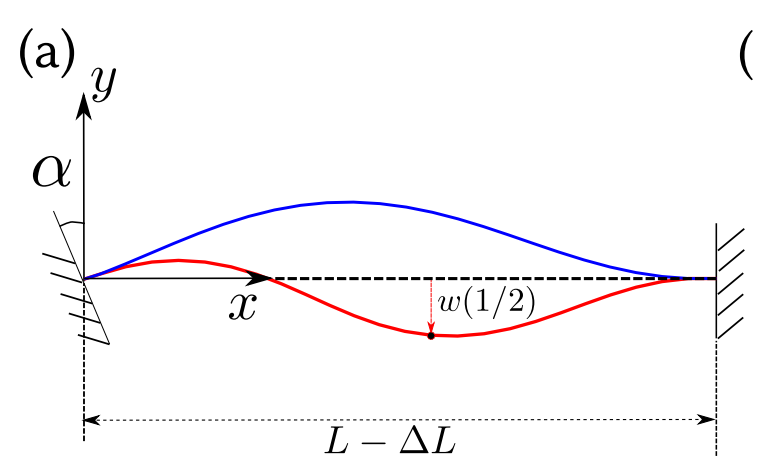

(b) $w^{w(1 / 2)(L \Delta L)^{-1 / 2}}$

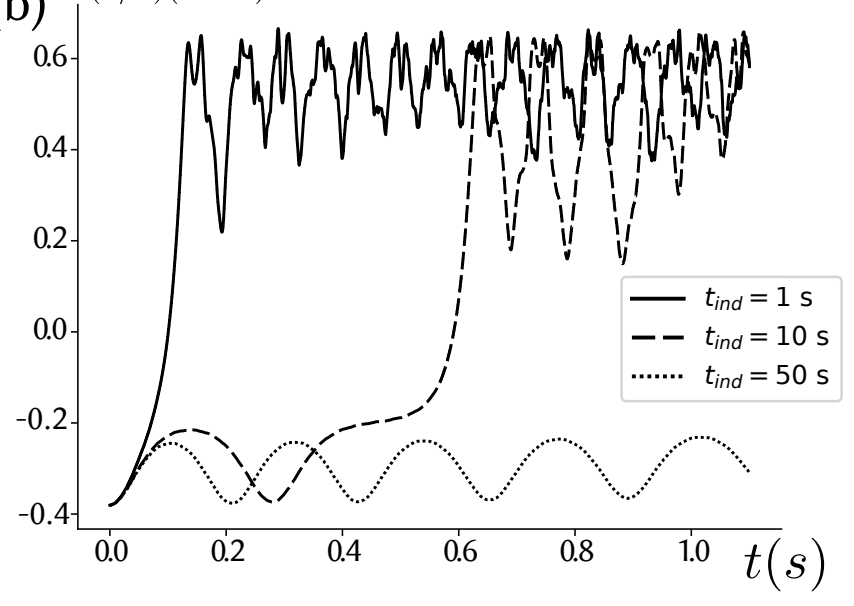

Figure 8.7: Dynamics of visco-elastic snap-through. (a) Initial equilibrium of a visco-elastic arch of undeformed length $L=100$ with clamping angle $\alpha=0.35$ and end-to-end shortening $\Delta L=2.8$ (in blue); intermediate configuration obtained by applying a midpoint constraint $w(1 / 2)=0.38 \sqrt{L \Delta L}$, held fixed for a variable duration $t_{\text {ind }}$ (in red). (b) Vertical displacement of the midpoint of the beam, following its release at time $t=0$. The time interval $t_{\text {ind }}$ during which the midpoint constraint has been applied determines the long-time behavior of the arch. 
the Newmark scheme. The beam has a density of $\rho=7.210^{-8}$, a thickness $h=0.1$, and an elastic modulus $E_{2}=6.10^{3}$ and the other parameters are adjusted as to have $\mathrm{De}=10$ and $\beta=0.1$. Figure 8.7 (b) shows the scaled vertical position of the midpoint, $w(1 / 2)(L \Delta L)^{-1 / 2}$ as a function of time following the release of the midpoint, for different values of the initial indentation time $t_{\text {ind }}=1 \mathrm{~s}, t_{\text {ind }}=10 \mathrm{~s}$, and $t_{\text {ind }}=50 \mathrm{~s}$. When the indentation time is sufficiently large, a pseudo-bistable behavior is observed, as discussed by Gomez et al. (2019).

\section{Conclusion}

We have introduced a unified numerical framework for modeling slender beams in a geometrically exact way, which is applicable to a wide range of constitutive models, including rate-dependent material behavior. We build on the geometric description of discrete framed curves, which is borrowed from the computer graphics community (Bergou et al., 2008, 2010; Jawed et al., 2017). In our approach, the beam geometry is fully decoupled from the constitutive behavior, which results in a versatile method which can easily be applied to a variety of constitutive models for the underlying base material. We have demonstrated the versatility of our approach by showcasing benchmark problems that involve linear elastic, viscous and visco-elastic materials. Our numerical examples further include nonlinear quasistatic problems (solved by continuation using Newton's method at each load increment) and dynamic problems (involving both modal analysis and the solution of transient responses by implicit time integration). Through an incremental formulation based on variational constitutive updates, we also described rate-dependent material behavior, as demonstrated for viscous and visco-elastic beams. This formulation can further be adapted to model history-dependent irreversible behavior such as plasticity or visco-plasticity as well as more complex constitutive laws such as active materials or metabeams (Coulais et al., 2015). This combination of a geometrically exact discrete beam description with a time-discrete variational approach is the main novelty of our implementation: we demonstrate its efficiency based on a specific discretization of Cosserat rods. However, it could potentially be combined with other discrete beam models. With our choice of discretization, we reported quadratic convergence for problems involving bending and twist and linear convergence for pure stretching; this is comparable to the convergence achieved by alternative formulations (Crisfield, 1990). Our implementation yields sparse or banded stiffness matrices and embeds the unshearability constraint. Extensions of this implementation to beam assemblies and trusses with rigid joints will be the subject of future work.

\section{Acknowledgments}

D.M.K. gratefully acknowledge the support from the U.S. Office of Naval Research (ONR) through award N00014-16-1-2431. C.L. acknowledges support through an ETH Postdoctoral Fellowship. We thank Manuel Weberndorfer for fruitful discussions on the numerical implementation.

\section{Appendix A. Area of a spherical triangle and variation of the holonomy}

\section{Appendix A.1. Explicit calculation of the area of a spherical triangle}

The computation of the holonomy introduced in Section 3.3 requires the evaluation of the area of two spherical triangles drawn on the unit sphere. The area $\mathcal{A}$ of a spherical triangle spanned by the unit vectors $\left(\mathbf{v}_{1}, \mathbf{v}_{2}, \mathbf{v}_{3}\right)$ can be calculated from the sum of the three angles $A, B, C$, depicted in Figure A.1, as

$$
\mathcal{A}\left(\mathbf{v}_{1}, \mathbf{v}_{2}, \mathbf{v}_{3}\right)=A+B+C-\pi
$$

The angles $A, B$ and $C$ are obtained as follows. We first define

$$
\begin{gathered}
\cos a=\mathbf{v}_{2} \cdot \mathbf{v}_{3}, \quad \cos b=\mathbf{v}_{3} \cdot \mathbf{v}_{1}, \quad \cos c=\mathbf{v}_{2} \cdot \mathbf{v}_{1}, \\
\sin a=\sqrt{1-\cos ^{2} a}, \quad \sin b=\sqrt{1-\cos ^{2} b}, \quad \sin c=\sqrt{1-\cos ^{2} c} .
\end{gathered}
$$




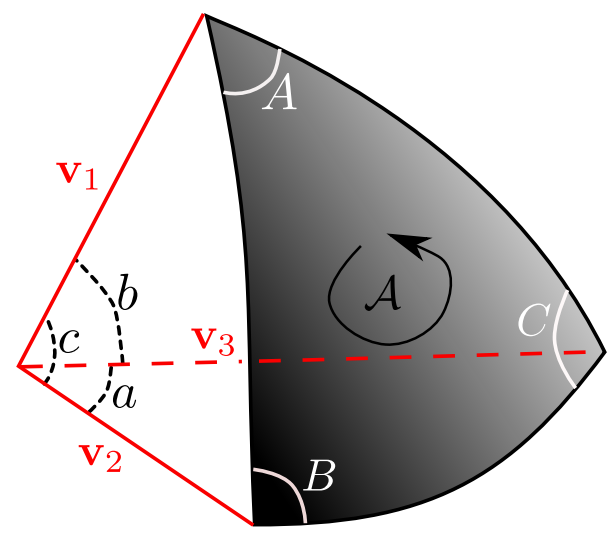

Figure A.1: Caculation of the area of a spherical triangle.

Then, we use spherical trigonometry to obtain

$$
\cos A=\frac{\cos a-\cos b \cos c}{\sin b \sin c}, \quad \cos B=\frac{\cos b-\cos c \cos a}{\sin c \sin a}, \quad \cos C=\frac{\cos c-\cos a \cos b}{\sin a \sin b},
$$

and

$$
\sin A=\frac{\left(\mathbf{v}_{1} \times \mathbf{v}_{2}\right) \cdot \mathbf{v}_{3}}{\sin b \sin c}, \quad \sin B=\frac{\left(\mathbf{v}_{1} \times \mathbf{v}_{2}\right) \cdot \mathbf{v}_{3}}{\sin c \sin a}, \quad \sin C=\frac{\left(\mathbf{v}_{1} \times \mathbf{v}_{2}\right) \cdot \mathbf{v}_{3}}{\sin a \sin b} .
$$

We calculate the angles $a, b$ and $c$, and then $A, B$ and $C$ using the 2-arguments arctangent function, which allows to pick the correct determination: the arguments passed to the arctangent function are the cosines and sines given by the formulas below.

Like the expression of parallel transport, the expression of the area of the spherical triangle $\mathcal{A}\left(\mathbf{v}_{1}, \mathbf{v}_{2}, \mathbf{v}_{3}\right)$ given by Eqs. (A.1a) to (A.1d) becomes ill-determined when two of the three vectors are colinear, whereas the value of the area tends to 0 . When the vectors are nearly equal, we replace the previous, general expression of $\mathcal{A}$ with the expansion

$$
\mathcal{A}\left(\mathbf{v}_{1}, \mathbf{v}_{2}, \mathbf{v}_{3}\right) \approx \frac{\mathbf{v}_{1} \times \mathbf{v}_{2}}{1+\mathbf{v}_{1} \cdot \mathbf{v}_{2}} \cdot \mathbf{v}_{3}\left(1-\frac{\mathbf{v}_{1} \cdot\left(\mathbf{v}_{3}-\mathbf{v}_{2}\right)}{2\left(1+\mathbf{v}_{1} \cdot \mathbf{v}_{2}\right)}+\frac{\left(\mathbf{v}_{1} \cdot\left(\mathbf{v}_{3}-\mathbf{v}_{2}\right)\right)^{2}-\frac{\left(1+\mathbf{v}_{1} \cdot \mathbf{v}_{2}\right)\left(2+\mathbf{v}_{1} \cdot \mathbf{v}_{2}\right)\left(\mathbf{v}_{2} \cdot \mathbf{v}_{3}-1\right)}{1+\mathbf{v}_{2} \cdot \mathbf{v}_{3}}}{3\left(1+\mathbf{v}_{1} \cdot \mathbf{v}_{2}\right)^{2}}\right) .
$$

In practice, we replace the general expression by the above approximate form when $\left|\mathbf{v}_{3}-\mathbf{v}_{2}\right|<0.001$. Similar approximations for $\mathcal{A}\left(\mathbf{v}_{1}, \mathbf{v}_{2}, \mathbf{v}_{3}\right)$ when $\mathbf{v}_{3} \approx \mathbf{v}_{1}$ and when $\mathbf{v}_{1} \approx \mathbf{v}_{2}$ can easily be defined by circular permutation.

\section{Appendix A.2. First variation of the area of a spherical triangle}

The area of an infinitesimal spherical triangle spanned by $\left(\mathbf{v}_{1}, \mathbf{v}_{2}, \mathbf{v}_{2}+\delta \mathbf{v}_{2}\right)$ is

$$
\mathcal{A}\left(\mathbf{v}_{1}, \mathbf{v}_{2}, \mathbf{v}_{2}+\delta \mathbf{v}_{2}\right)=\frac{\mathbf{v}_{1} \times \mathbf{v}_{2}}{1+\mathbf{v}_{1} \cdot \mathbf{v}_{2}} \cdot \delta \mathbf{v}_{2}+\mathcal{O}\left(\left|\mathbf{v}_{2}\right|^{2}\right) .
$$

\section{Appendix A.3. First variation of the holonomy}

We decompose the variation of the holonomy (4.7), associated with the increments $\delta \mathbf{t}^{i-1}$ and $\delta \mathbf{t}^{i}$, into four spherical triangles sketched in Figure A.2. Using (A.2) to calculate the areas of the spherical triangles highlighted in white and green in Figure A.2(a), we express the difference between the gray and red areas in Figure A.2 as

$$
\begin{aligned}
\mathcal{A}\left(\mathbf{t}_{\star}^{i-1}, \mathbf{t}_{\star}^{i}, \mathbf{t}^{i}+\delta \mathbf{t}^{i}, \mathbf{t}^{i-1}+\delta \mathbf{t}^{i-1}\right)-\gamma_{i} & =-\frac{\mathbf{t}_{\star}^{i} \times \mathbf{t}^{i}}{1+\mathbf{t}_{\star}^{i} \cdot \mathbf{t}^{i}} \cdot \delta \mathbf{t}^{i}+\frac{\mathbf{k}_{i}}{2} \cdot\left(\delta \mathbf{t}^{i-1}+\delta \mathbf{t}^{i}\right)-\frac{\mathbf{t}^{i-1} \times \mathbf{t}_{\star}^{i-1}}{1+\mathbf{t}^{i-1} \cdot \mathbf{t}_{\star}^{i-1}} \cdot \delta \mathbf{t}^{i-1} \\
& =\frac{\delta \mathbf{t}^{i-1}}{2} \cdot\left(\mathbf{b}_{\star}^{i-1}+\mathbf{k}_{i}\right)+\frac{\delta \mathbf{t}^{i}}{2} \cdot\left(-\mathbf{b}_{\star}^{i}+\mathbf{k}_{i}\right) .
\end{aligned}
$$

This difference is the variation of the holonomy. 
(a)

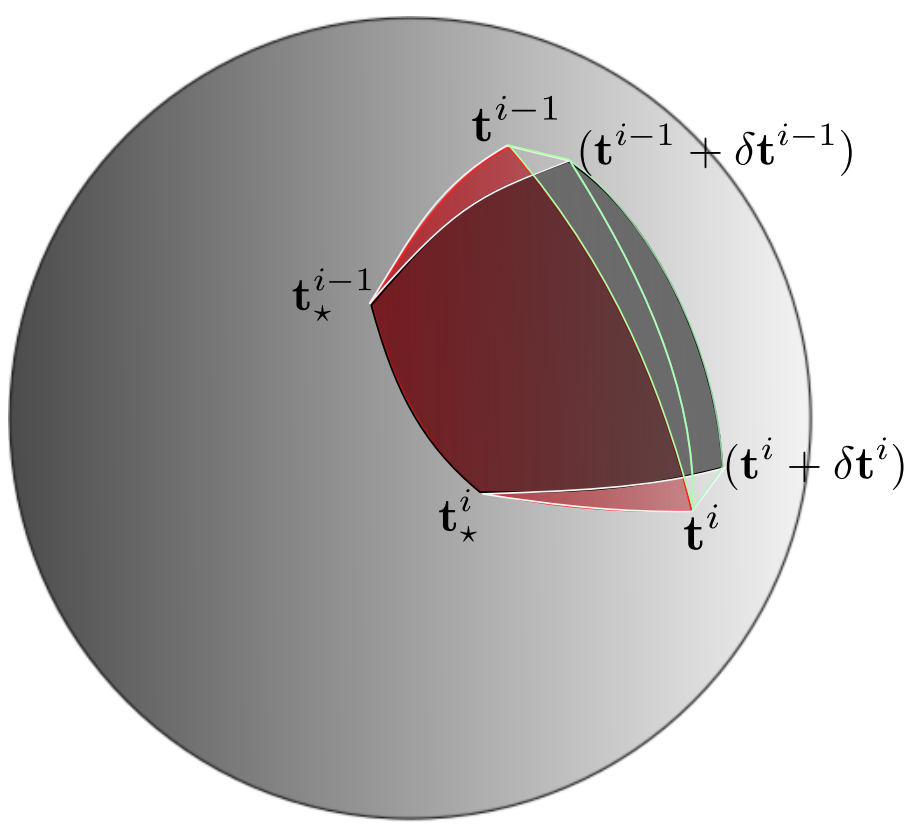

(b) $\quad \mathbf{t}^{i-1} \quad \mathbf{t}^{i-1}\left(\mathrm{t}^{i-1}+\delta \mathbf{t}^{i-1}\right) \quad\left(\mathbf{t}^{i-1}+\delta \mathbf{t}^{i-1}\right)$

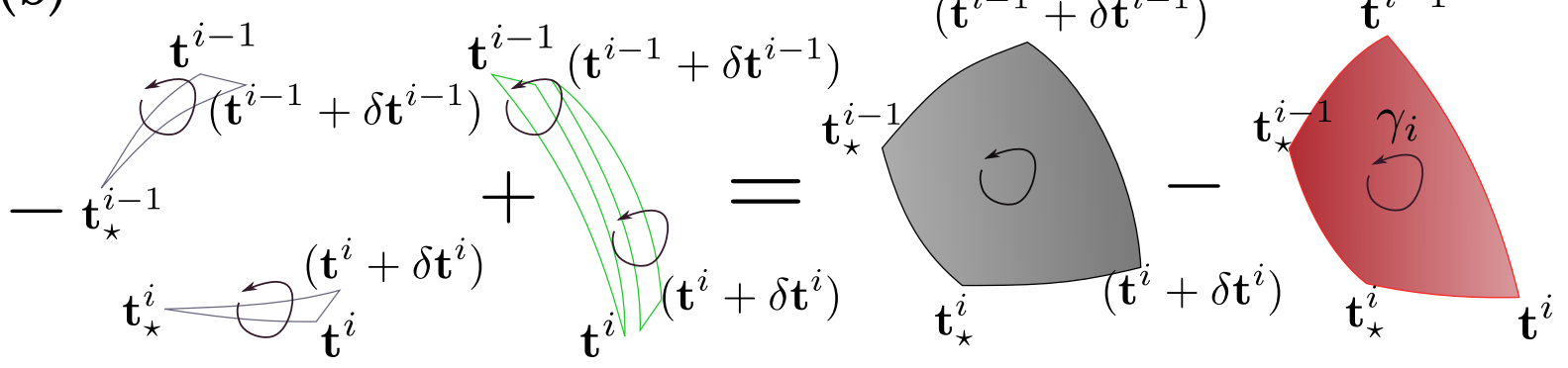

Figure A.2: Variation of the holonomy. (a) Variation of the holonomy on the unit sphere (difference between areas in dark gray and in red), whose individual contributions are the spherical triangles highlighted in white and green. (b) Decomposition of the signed areas from (a). 


\section{Appendix B. Second variations of strains}

Appendix B.1. Second variation of unit tangents and material frame vectors

The second variation of a unit tangents is given by $\delta^{2} \mathbf{t}^{i \pm}=\delta \mathbf{u}_{i}^{1} \cdot \mathrm{D}^{2} \mathbf{t}^{i \pm} \cdot \delta \mathbf{u}_{i}^{2}$. To avoid any ambiguity on the indices of this third-order tensor, we prefer to focus on the second variation of the kth-component of the unit tangent in the Cartesian frame, $\left(\delta^{2} \mathbf{t}^{i \pm}\right)_{k}=\delta \mathbf{u}^{1} \cdot\left(\mathrm{D}^{2} \mathbf{t}^{i \pm}\right)_{k} \cdot \delta \mathbf{u}^{2}$, where $\delta \mathbf{u}^{1}$ and $\delta \mathbf{u}^{2}$ are independent infinitesimal variation. Using (5.4), we find

$$
\left(\mathrm{D}^{2} \mathbf{t}^{i \pm}\right)_{k}=-\frac{\left(\mathbf{e}^{i \pm}\right)_{k}}{\left\|\mathbf{e}^{i \pm}\right\|^{3}} \mathcal{M}_{k}+\frac{3\left(\mathbf{e}^{i \pm}\right)_{k}\left(\mathbf{e}^{i \pm} \otimes \mathbf{e}^{i \pm}\right)}{\left\|\mathbf{e}^{i \pm}\right\|^{5}}, \quad \text { for } \quad k \in\{1,2,3\}
$$

where

$$
\mathcal{M}_{1}=\left(\begin{array}{lll}
3 & 1 & 1 \\
1 & 1 & 0 \\
1 & 0 & 1
\end{array}\right), \quad \mathcal{M}_{2}=\left(\begin{array}{lll}
1 & 1 & 0 \\
1 & 3 & 1 \\
0 & 1 & 1
\end{array}\right), \quad \mathcal{M}_{3}=\left(\begin{array}{lll}
1 & 0 & 1 \\
0 & 1 & 1 \\
1 & 1 & 3
\end{array}\right)
$$

Using this expression, the second variation of the material frame vectors $\mathbf{d}_{J}^{i \pm}$ is derived from Eq. (5.7) and writes, for $I \in\{1,2\}$,

$$
\begin{aligned}
& \delta^{2} \mathbf{d}_{I}^{i \pm}=-\delta \omega_{1}^{i \pm} \delta \omega_{2}^{i \pm} \mathbf{d}_{I}^{i}+\delta \omega_{1}^{i \pm}\left(\mathbf{d}_{I}^{i \pm} \otimes \frac{\mathbf{b}_{\star}^{i \pm}}{2}-\sum_{J=1}^{2} \eta_{I J} \mathbf{t}^{i \pm} \otimes \mathbf{d}_{J}^{i \pm}\right) \cdot \delta \mathbf{t}_{2}^{i \pm} \\
&+\delta \omega_{2}^{i \pm}\left(\mathbf{d}_{I}^{i \pm} \otimes \frac{\mathbf{b}_{\star}^{i \pm}}{2}-\sum_{J=1}^{2} \eta_{I J} \mathbf{t}^{i \pm} \otimes \mathbf{d}_{J}^{i \pm}\right) \cdot \delta \mathbf{t}_{1}^{i \pm}+\delta \mathbf{t}_{1}^{i \pm} \cdot \mathrm{D}_{22}^{2} \mathbf{d}_{I}^{i} \cdot \delta \mathbf{t}_{2}^{i \pm} \\
&-\left[\sum_{J=1}^{2} \eta_{I J} \mathbf{d}_{J}^{i \pm} \otimes \frac{\mathbf{b}_{\star}^{i \pm}}{2}+\mathbf{t}^{i \pm} \otimes \mathbf{d}_{I}^{i \pm}\right] \cdot \delta^{2} \mathbf{t}^{i \pm}
\end{aligned}
$$

where the subscript indices 1 and 2 refer respectively to the two increments of displacement $\delta \mathbf{u}^{1}$ and $\delta \mathbf{u}^{2}$ considered in the calculation of the second variation. In this expression, the operator $\mathrm{D}_{22}^{2} \mathbf{d}_{I}^{i}$ defines the second order partial derivative of the material frame vector $\mathbf{d}_{I}^{i}$ with respect to the unit vector $\mathbf{t}^{i}$. It can be expressed as follows

$$
\begin{aligned}
\left(\mathrm{D}_{22}^{2} \mathbf{d}_{I}^{i \pm}\right)_{k} & =-\frac{\mathbf{b}_{\star}^{i \pm} \otimes \mathbf{b}_{\star}^{i \pm}}{4}\left(\mathbf{d}_{I}^{i \pm}\right)_{k} \\
& +\left[\left(\mathbf{d}_{I}^{i \pm} \otimes \mathbf{t}^{i \pm}+\sum_{J=1}^{2} \eta_{I J} \mathbf{b}_{\star}^{i \pm} \otimes \mathbf{d}_{J}^{i \pm}\right)\left(\mathbf{t}^{i \pm}\right)_{k}+\mathbf{e}_{k} \otimes \mathbf{d}_{I}^{i \pm}-\sum_{J=1}^{2} \frac{\mathrm{D}_{2} \mathbf{b}_{\star}^{i \pm}}{2} \eta_{I J}\left(\mathbf{d}_{J}^{i \pm}\right)_{k}\right]_{\mathrm{sym}},
\end{aligned}
$$

with $[\mathcal{T}]_{\text {sym }}=\frac{\mathcal{T}^{T}+\mathcal{T}}{2}$ denoting the symmetric part of a matrix $\mathcal{T}$, and $\left(\mathbf{e}_{k}\right)_{k \in\{1,2,3\}}$ denoting the director's frame of the Cartesian basis in which all quantities are expressed, not to be confused with the notation for segment vectors (index is in superscript).

\section{Appendix B.2. Second variation for bending}

Using the expression (B.1), we write the second variation of the binormal vector $\mathbf{k}_{i}\left(\mathbf{t}^{i-1}, \mathbf{t}^{i}\right)$ as

$$
\delta^{2} \mathbf{k}_{i}=\left[\begin{array}{c}
\delta \mathbf{t}_{1}^{i-1} \\
\delta \mathbf{t}_{1}^{i}
\end{array}\right] \cdot \mathrm{D}^{2} \mathbf{k}_{i} \cdot\left[\begin{array}{c}
\delta \mathbf{t}_{2}^{i-1} \\
\delta \mathbf{t}_{2}^{i}
\end{array}\right]+\mathrm{D}_{1} \mathbf{k}_{i} \cdot \delta^{2} \mathbf{t}^{i-1}+\mathrm{D}_{2} \mathbf{k}_{i} \cdot \delta^{2} \mathbf{t}^{i}
$$

where $\mathrm{D}_{1} \mathbf{k}_{i}$ and $\mathrm{D}_{2} \mathbf{k}_{i}$ are defined in (5.9) and $\mathrm{D}^{2} \mathbf{k}_{i}=\mathrm{D}^{2} \mathbf{b}\left(\mathbf{t}^{i-1}, \mathbf{t}^{i}\right)$ is the generic second variation of the binormal vector of two unit vectors $\mathbf{v}_{1}$ and $\mathbf{v}_{2}$. The later can be decomposed as

$$
\mathrm{D}^{2} \mathbf{b}\left(\mathbf{v}_{1}, \mathbf{v}_{2}\right)=\left(\begin{array}{cc}
\mathrm{D}_{11}^{2} \mathbf{b}\left(\mathbf{v}_{1}, \mathbf{v}_{2}\right) & \mathrm{D}_{12}^{2} \mathbf{b}\left(\mathbf{v}_{1}, \mathbf{v}_{2}\right) \\
\left(\mathrm{D}_{12}^{2} \mathbf{b}\left(\mathbf{v}_{1}, \mathbf{v}_{2}\right)\right)^{T} & \mathrm{D}_{22}^{2} \mathbf{b}\left(\mathbf{v}_{1}, \mathbf{v}_{2}\right)
\end{array}\right)
$$


with

$$
\begin{gathered}
\left(\mathrm{D}_{11}^{2} \mathbf{b}\left(\mathbf{v}_{1}, \mathbf{v}_{2}\right)\right)_{k}=2\left[\frac{\left(\mathbf{v}_{2} \times\right)^{T} \cdot\left(\mathbf{e}_{k} \otimes \mathbf{v}_{2}\right)+\left(\mathbf{e}_{k} \otimes \mathbf{v}_{2}\right)^{T} \cdot \mathbf{v}_{2 \times}}{\left(1+\mathbf{v}_{1} \cdot \mathbf{v}_{2}\right)^{2}}+2 \frac{\left(\mathbf{v}_{1} \times \mathbf{v}_{2}\right)_{k} \mathbf{v}_{2} \otimes \mathbf{v}_{2}}{\left(1+\mathbf{v}_{1} \cdot \mathbf{v}_{2}\right)^{3}}\right], \\
\left(D_{22}^{2} \mathbf{b}\left(\mathbf{v}_{1}, \mathbf{v}_{2}\right)\right)_{k}=2\left[-\frac{\left(\mathbf{v}_{1} \times\right)^{T} \cdot\left(\mathbf{e}_{k} \otimes \mathbf{v}_{1}\right)+\left(\mathbf{e}_{k} \otimes \mathbf{v}_{1}\right)^{T} \cdot \mathbf{v}_{1 \times}}{\left(1+\mathbf{v}_{1} \cdot \mathbf{v}_{2}\right)^{2}}+2 \frac{\left(\mathbf{v}_{1} \times \mathbf{v}_{2}\right)_{k} \mathbf{v}_{1} \otimes \mathbf{v}_{1}}{\left(1+\mathbf{v}_{1} \cdot \mathbf{v}_{2}\right)^{3}}\right], \\
\left(D_{12}^{2} \mathbf{b}\left(\mathbf{v}_{1}, \mathbf{v}_{2}\right)\right)_{k}=2 \frac{-\left(\mathbf{v}_{1 \times}\right)^{T} \cdot\left(\mathbf{e}_{k} \otimes \mathbf{v}_{2}\right)+\left(\mathbf{e}_{k} \otimes \mathbf{v}_{1}\right)^{T} \cdot \mathbf{v}_{2} \times \mathbf{1}_{3}\left(\mathbf{v}_{2} \times \mathbf{v}_{1}\right)_{k}}{\left(1+\mathbf{v}_{1} \cdot \mathbf{v}_{2}\right)^{2}} \\
-2 \frac{\mathcal{C}_{k}}{1+\mathbf{v}_{1} \cdot \mathbf{v}_{2}}+4 \frac{\left(\mathbf{v}_{1} \times \mathbf{v}_{2}\right)_{k} \mathbf{v}_{1} \otimes \mathbf{v}_{2}}{\left(1+\mathbf{v}_{1} \cdot \mathbf{v}_{2}\right)^{3}},
\end{gathered}
$$

where $\mathcal{C}_{1}=-\mathbf{e}_{3} \otimes \mathbf{e}_{2}+\mathbf{e}_{2} \otimes \mathbf{e}_{3}, \mathcal{C}_{2}=\mathbf{e}_{3} \otimes \mathbf{e}_{1}-\mathbf{e}_{1} \otimes \mathbf{e}_{3}$ and $\mathcal{C}_{3}=-\mathbf{e}_{2} \otimes \mathbf{e}_{1}+\mathbf{e}_{1} \otimes \mathbf{e}_{2}$. In view of this, the second variation of the bending strains becomes, for $I \in\{1,2\}$,

$$
\begin{aligned}
\delta^{2} \kappa_{i}^{I}=\delta^{2} \mathbf{k}_{i} \cdot \frac{\mathbf{d}_{I}^{i-1}+\mathbf{d}_{I}^{i}}{2}+\frac{\mathbf{k}_{i}}{2} \cdot\left(\delta^{2} \mathbf{d}_{I}^{i-1}+\right. & \left.\delta^{2} \mathbf{d}_{I}^{i}\right) \\
+ & \frac{1}{2}\left(\mathrm{D}_{1} \mathbf{k}_{i} \cdot \delta \mathbf{t}_{1}^{i-1}+\right. \\
& \left.\mathrm{D}_{2} \mathbf{k}_{i} \cdot \delta \mathbf{t}_{1}^{i}\right) \cdot\left(\mathrm{Dd}_{I}^{i-1} \cdot \delta \mathbf{t}_{2}^{i-1}+\mathrm{Dd}_{I}^{i} \cdot \mathbf{t}_{2}^{i}\right) \\
& +\frac{1}{2}\left(\mathrm{D}_{1} \mathbf{k}_{i} \cdot \delta \mathbf{t}_{2}^{i-1}+\mathrm{D}_{2} \mathbf{k}_{i} \cdot \delta \mathbf{t}_{2}^{i}\right) \cdot\left(\mathrm{Dd}_{I}^{i-1} \cdot \delta \mathbf{t}_{1}^{i-1}+\mathrm{Dd}_{I}^{i} \cdot \mathbf{t}_{1}^{i}\right) .
\end{aligned}
$$

Appendix B.3. Second variation of the twist

Starting from Eq. (5.12), we derive the second variation of the twist as

$$
\delta^{2} \tau_{i}=\left[\begin{array}{c}
\delta \mathbf{t}_{1}^{i-1} \\
\delta \mathbf{t}_{1}^{i}
\end{array}\right] \cdot \mathrm{D}^{2} \gamma_{i} \cdot\left[\begin{array}{c}
\delta \mathbf{t}_{2}^{i-1} \\
\delta \mathbf{t}_{2}^{i}
\end{array}\right]+\frac{\mathbf{k}_{i}+\mathbf{b}_{\star}^{i-1}}{2} \cdot \delta^{2} \mathbf{t}^{i-1}+\frac{\mathbf{k}_{i}-\mathbf{b}_{\star}^{i}}{2} \cdot \delta^{2} \mathbf{t}^{i},
$$

with $\mathrm{D}^{2} \gamma_{i}=\left[\begin{array}{cc}\mathrm{D}_{11}^{2} \gamma_{i} & \mathrm{D}_{12}^{2} \gamma_{i} \\ \left(\mathrm{D}_{12}^{2} \gamma_{i}\right)^{T} & \mathrm{D}_{22}^{2} \gamma_{i}\end{array}\right]$ where

$$
\mathrm{D}_{11}^{2} \gamma_{i}=\frac{1}{2}\left[\mathrm{D}_{2} \mathbf{b}_{\star}^{i-1}+\mathrm{D}_{1} \mathbf{k}_{i}\right]_{\mathrm{sym}}, \quad \mathrm{D}_{22}^{2} \gamma_{i}=\frac{1}{2}\left[-\mathrm{D}_{2} \mathbf{b}_{\star}^{i}+\mathrm{D}_{2} \mathbf{k}_{i}\right]_{\mathrm{sym}}, \quad \mathrm{D}_{12}^{2} \gamma_{i}=\frac{1}{4}\left(\left(\mathrm{D}_{1} \mathbf{k}_{i}\right)^{T}+\mathrm{D}_{2} \mathbf{k}_{i}\right),
$$

and

$$
\mathrm{D}_{2} \mathbf{b}_{\star}^{i-1}=\mathrm{D}_{2} \mathbf{b}\left(\mathbf{t}_{\star}^{i-1}, \mathbf{t}^{i-1}\right), \quad \mathrm{D}_{2} \mathbf{b}_{\star}^{i}=\mathrm{D}_{2} \mathbf{b}\left(\mathbf{t}_{\star}^{i}, \mathbf{t}^{i}\right), \quad \mathrm{D}_{1} \mathbf{k}_{i}=\mathrm{D}_{1} \mathbf{b}\left(\mathbf{t}^{i-1}, \mathbf{t}^{i}\right), \quad \text { and } \quad \mathrm{D}_{2} \mathbf{k}_{i}=\mathrm{D}_{2} \mathbf{b}\left(\mathbf{t}^{i-1}, \mathbf{t}^{i}\right) .
$$

\section{Appendix C. Definition of the discrete mass matrices}

We denote by $A_{0}$ the cross-sectional area of the beam, by $I_{0}$ its rotational moment of inertia, and by $l_{0}^{i}$ the length of segment $i$. We consider a small axial strain $|\varepsilon| \ll 1$, so that all those quantities remain approximately constant. The kinetic energy of a discrete beam, cf. Eq. (7.1), is approximated by a sum over all segments, i.e.,

$$
\mathcal{E}_{c}^{\text {discrete }}=\frac{\rho}{2} \sum_{i=0}^{n-2}\left(A_{0} l_{0}^{i}\left(\frac{\dot{\mathbf{x}}_{i}+\dot{\mathbf{x}}_{i+1}}{2}\right)^{2}+I_{0}\left(\theta^{i}\right)^{2}\right)=\frac{1}{2} \mathbf{v} \cdot \mathbf{M} \cdot \mathbf{v},
$$

where the consistent mass matrix $\mathbf{M}$ can be decomposed into

$$
\mathbf{M}=\sum_{i=1}^{n-2} \mathbf{C}_{i}^{T} \cdot \mathbf{M}_{i} \cdot \mathbf{C}_{i}
$$


which depends on the connectivity matrices $\left(\mathbf{C}_{i}\right)_{i \in\{1, \cdots, n-2\}}$ and the consistent mass matrix $\mathbf{M}_{i}$ of element $i$, viz.

$$
\begin{aligned}
& \mathbf{M}_{i}=\rho\left(\begin{array}{ccccc}
\mathbf{0} & \mathbf{0} & \mathbf{0} & 0 & 0 \\
& A_{0} \frac{l_{0}^{i-1}+l_{0}^{i}}{4} & \frac{A_{0} l_{0}^{i}}{4} \mathbf{1} & 0 & 0 \\
& & \mathbf{0} & 0 & 0 \\
& & & \frac{l_{0}^{i-1}}{2} I_{0} & 0 \\
& & & & \frac{l_{0}^{i}}{2} I_{0}
\end{array}\right) \quad \text { for } i \in\{2, \cdots, n-3\} \\
& \mathbf{M}_{1}=\rho\left(\begin{array}{ccccc}
\frac{A_{0} l_{0}^{0}}{4} \mathbf{1} & \frac{A_{0} l_{0}^{0}}{4} \mathbf{1} & \mathbf{0} & 0 & 0 \\
& A_{0} \frac{l_{0}^{0}+l_{0}^{1}}{4} \mathbf{1} & \frac{A_{0} l_{0}^{1}}{4} \mathbf{1} & 0 & 0 \\
& & \mathbf{0} & 0 & 0 \\
& \operatorname{sym} & & l_{0}^{0} I_{0} & 0 \\
& & & & \frac{l_{0}^{1}}{2} I_{0}
\end{array}\right) \\
& \mathbf{M}_{n-2}=\rho\left(\begin{array}{ccccc}
\mathbf{0} & \mathbf{0} & \mathbf{0} & 0 & 0 \\
& A_{0} \frac{l_{0}^{n-3}+l_{0}^{n-2}}{4} \mathbf{1} & \frac{A_{0} l_{0}^{n-2}}{4} \mathbf{1} & 0 & 0 \\
& & \frac{A_{0} l_{0}^{n-2}}{4} \mathbf{1} & 0 & 0 \\
& & & \frac{l_{0}^{n-3}}{2} I_{0} & 0 \\
& & & & l_{0}^{n-2} I_{0}
\end{array}\right) .
\end{aligned}
$$

It is convenient to also define a diagonal lumped mass matrix. To this end, we redefine the discrete kinetic energy as

$$
\tilde{\mathcal{E}}_{c}^{\text {discrete }}=\frac{\rho}{2}\left(\frac{A_{0} l_{0}^{0}}{2} \dot{\mathbf{x}}_{0}^{2}+\frac{A_{0} l_{0}^{n-2}}{2} \dot{\mathbf{x}}_{n-1}^{2}+\sum_{i=1}^{n-2}\left[A_{0} \frac{l_{0}^{i-1}+l_{0}^{i}}{2} \dot{\mathbf{x}}_{i}^{2}+I_{0} \frac{\left(\theta^{i}\right)^{2}+\left(\theta^{i-1}\right)^{2}}{2}\right]\right),
$$

which we rewrite as

$$
\tilde{\mathcal{E}}_{c}^{\text {discrete }}=\frac{1}{2} \mathbf{v} \cdot \tilde{\mathbf{M}} \cdot \mathbf{v} \quad \text { with } \quad \tilde{\mathbf{M}}=\sum_{i=1}^{n-2} \mathbf{C}_{i}^{T} \cdot \tilde{\mathbf{M}}_{i} \cdot \mathbf{C}_{i} .
$$

$\tilde{\mathbf{M}}_{i}$ is the lumped (diagonal) mass matrix of element $i(i \in\{1, \cdots, n-2\})$, which is defined as

$$
\tilde{\mathbf{M}}_{i}=\rho\left(\begin{array}{ccccc}
A_{0} l_{0}^{i-1} \delta_{(i-1) 0} \mathbf{1} & \mathbf{0} & \mathbf{0} & 0 & 0 \\
& A_{0} \frac{l_{0}^{i-1}+l_{0}^{i}}{2} \mathbf{1} & \mathbf{0} & 0 & 0 \\
& & A_{0} l_{0}^{i} \delta_{i(n-1)} \mathbf{1} & 0 & 0 \\
& \text { sym. } & & l_{0}^{i-1} I_{0} & 0 \\
& & & & l_{0}^{i} I_{0} \delta_{(n-2) i}
\end{array}\right) \text {. }
$$

\section{Appendix D. Definition of internal forces and tangent moduli}

Appendix D.1. Elastic beam

With the choice (6.2) of discrete energy density, the vector of internal axial forces and moments $\boldsymbol{\Sigma}_{i}(7.7)$ in Eq. (7.10) writes

$$
\boldsymbol{\Sigma}_{i}=\left(\frac{E A l_{0}^{i-1}}{2} \varepsilon^{i-1}, \frac{E A l_{0}^{i}}{2} \varepsilon^{i}, E I_{1} \tilde{\kappa}_{i}^{1}, E I_{2} \tilde{\kappa}_{i}^{2}, \mu J \tilde{\tau}_{i}\right) .
$$

The tangent matrix is the diagonal matrix

$$
\mathbf{K}_{i}=\left(\begin{array}{ccccc}
\frac{E A l_{0}^{i-1}}{2} & 0 & 0 & 0 & 0 \\
& \frac{E A l_{0}^{i}}{2} & 0 & 0 & 0 \\
& & \frac{E I_{1}}{\left(\tilde{l}_{i}\right)_{0}} & 0 & 0 \\
& \text { sym. } & & \frac{E I_{2}}{\left(\tilde{l}_{i}\right)_{0}} & 0 \\
& & & & \frac{\mu J}{\left(\tilde{l}_{i}\right)_{0}}
\end{array}\right) .
$$


Appendix D.2. Viscous beam with surface tension

The vectors of effective internal tractions and moments associated with the constitutive model in Eqs. (6.5) and (6.6) are given by

$$
\begin{gathered}
\boldsymbol{\Sigma}_{i}^{\dagger}=\gamma\left(\frac{1}{2} \sqrt{\frac{\pi V^{i-1}}{\left(l^{i-1}\right)^{3}}}\left(l_{0}^{i-1}\right)^{2}, \frac{1}{2} \sqrt{\frac{\pi V^{i}}{\left(l^{i}\right)^{3}}}\left(l_{0}^{i}\right)^{2}, 0,0,0\right), \\
\mathbf{N}_{i}^{\dagger}=\frac{\eta}{\Delta t}\left(\frac{3 V^{i-1}}{2}\left(\frac{l_{0}^{i-1}}{l_{\star}^{i-1}}\right)^{4} \Delta_{\star} \varepsilon^{i-1}, \frac{3 V^{i}}{2}\left(\frac{l_{0}^{i}}{l_{\star}^{i}}\right)^{4} \Delta_{\star} \varepsilon^{i}, \frac{3\left(\tilde{I}_{i}\right)_{\star}}{\left(\tilde{l}_{i}\right)_{\star}} \Delta_{\star} \kappa_{i}^{1}, \frac{3\left(\tilde{I}_{i}\right)_{\star}}{\left(\tilde{l}_{i}\right)_{\star}} \Delta_{\star} \kappa_{i}^{2}, \frac{2\left(\tilde{I}_{i}\right)_{\star}}{\left(\tilde{l}_{i}\right)_{\star}} \Delta_{\star} \tau_{i}\right),
\end{gathered}
$$

where $\Delta_{\star} f=f-f_{\star}$. The matrix of effective tangent moduli writes

$$
\begin{aligned}
& \mathbf{K}_{i}^{\dagger}=\left(\begin{array}{ccccc}
-\frac{3 \gamma}{4} \sqrt{\frac{\pi V^{i-1}}{\left(l^{i-1}\right)^{7}}}\left(l_{0}^{i-1}\right)^{4} & 0 & 0 & 0 & 0 \\
& -\frac{3 \gamma}{4} \sqrt{\frac{\pi V^{i}}{\left(l^{i}\right)^{7}}}\left(l_{0}^{i}\right)^{4} & 0 & 0 & 0 \\
\text { sym. } & 0 & 0 & 0 \\
& & 0 & 0 \\
& & & 0
\end{array}\right) \\
& +\frac{\eta}{\Delta t}\left(\begin{array}{ccccc}
\frac{3 V^{i-1}}{2}\left(\frac{l_{0}^{i-1}}{l_{\star}^{i-1}}\right)^{4} & 0 & 0 & 0 & 0 \\
& \frac{3 V^{i}}{2}\left(\frac{l_{0}^{i}}{l_{\star}^{i}}\right)^{4} & 0 & 0 & 0 \\
& & \frac{3\left(\tilde{I}_{i}\right)_{\star}}{\left(\tilde{l}_{i}\right)_{\star}} & 0 & 0 \\
& & & \frac{3\left(\tilde{I}_{i}\right)_{\star}}{\left(\tilde{l}_{i}\right)_{\star}} & 0 \\
& & & & \frac{2\left(\tilde{I}_{i}\right)_{\star}}{\left(\tilde{l}_{i}\right)_{\star}}
\end{array}\right) .
\end{aligned}
$$

\section{Appendix D.3. Visco-elastic beam}

For the potential (6.8), the discrete kinetic evolution law (7.14) becomes

$$
-E_{2}\left(\kappa_{i}^{1}-z_{i}\right)+\eta \frac{z_{i}-z_{i}^{\star}}{\Delta t}=0
$$

which can be re-written as

$$
z_{i}=\frac{\eta / \Delta t}{\eta / \Delta t+E_{2}} z_{i}^{\star}+\frac{E_{2}}{\eta / \Delta t+E_{2}} \kappa_{i}^{1}
$$

Inserting this expression into $W_{i}$ and $\mathcal{D}_{i}$ in Eq. (6.8) yields

$$
\begin{gathered}
W_{i}^{\dagger}\left(\mathbf{E}_{i}\right)=\frac{I}{2\left(\tilde{l}_{i}\right)_{0}}\left[E_{1} \kappa_{i}^{1^{2}}+E_{2}\left(\kappa_{i}^{1}\left(1-\frac{E_{2}}{\eta / \Delta t+E_{2}}\right)-\frac{\eta / \Delta t}{\eta / \Delta t+E_{2}} z_{i}^{\star}\right)^{2}\right], \\
\mathcal{D}_{i}^{\dagger}\left(\mathbf{E}_{i}\right)=\frac{\eta I}{2\left(\tilde{l}_{i}\right)_{0} \Delta t^{2}}\left(\left(\frac{\eta / \Delta t}{\eta / \Delta t+E_{2}}-1\right) z_{i}^{\star}+\frac{E_{2}}{\eta / \Delta t+E_{2}} \kappa_{i}^{1}\right)^{2} .
\end{gathered}
$$

Note that we do not detail the contribution of the axial strain. Its associated energy in the potential (6.8) is elastic and thus yields a contribution to the internal forces and to the matrix of tangent moduli similar to the one found in Appendix D.1. The contributions asssociated with bending finally write

$$
\begin{gathered}
\frac{\partial W_{i}^{\dagger}}{\partial \kappa_{i}^{1}}=\frac{I}{\left(\tilde{l}_{i}\right)_{0}}\left[E_{2}\left(1-\frac{E_{2}}{\eta / \Delta t+E_{2}}\right)\left(\left(1-\frac{E_{2}}{\eta / \Delta t+E_{2}}\right) \kappa_{i}^{1}-\frac{\eta / \Delta t}{\eta / \Delta t+E_{2}} z_{i}^{\star}\right)+E_{1} \kappa_{i}^{1}\right] \\
\frac{\partial^{2} W_{i}^{\dagger}}{\partial \kappa_{i}^{1}}=\frac{I}{\left(\tilde{l}_{i}\right)_{0}}\left[E_{2}\left(1-\frac{E_{2}}{\eta / \Delta t+E_{2}}\right)^{2}+E_{1}\right]
\end{gathered}
$$




$$
\begin{gathered}
\frac{\partial \mathcal{D}_{i}^{\dagger}}{\partial \kappa_{i}^{1}}=\frac{\eta I}{\left(\tilde{l}_{i}\right)_{0} \Delta t^{2}}\left[\frac{E_{2}}{\eta / \Delta t+E_{2}}\left(\frac{E_{2}}{\eta / \Delta t+E_{2}} \kappa_{i}^{1}+\left(\frac{\eta / \Delta t}{\eta / \Delta t+E_{2}}-1\right) z_{i}^{\star}\right)\right], \\
\frac{\partial^{2} \mathcal{D}_{i}^{\dagger}}{\partial \kappa_{i}^{1^{2}}}=\frac{\eta I}{\left(\tilde{l}_{i}\right)_{0} \Delta t^{2}}\left[\frac{E_{2}}{\eta / \Delta t+E_{2}}\right]^{2} .
\end{gathered}
$$

Similar discrete constitutive laws can be derived for a 3D visco-elastic beam undergoing twist and bending.

J. Aldinger, I. Klapper, and M. Tabor. Formulae for the calculation and estimation of writhe. Journal of Knot theory and Its Ramifications, 4:343-372, 1995.

B. Audoly and Y. Pomeau. Elasticity and geometry: from hair curls to the nonlinear response of shells. Oxford University Press, 2010.

B. Audoly, N. Clauvelin, P.-T. Brun, M. Bergou, E. Grinspun, and M. Wardetzky. A discrete geometric approach for simulating the dynamics of thin viscous threads. Journal of Computational Physics, 253:18-49, 2013.

A. B. Basset. On the deformation of thin elastic wires. American Journal of Mathematics, 17:281-317, 1895.

M. Bergou, M. Wardetzky, S. Robinson, B. Audoly, and E. Grinspun. Discrete elastic rods. ACM transactions on graphics, $27: 63,2008$.

M. Bergou, B. Audoly, E. Vouga, M. Wardetzky, and E. Grinspun. Discrete viscous threads. ACM Transactions on Graphics, 29, 2010

K. Bertoldi, V. Vitelli, J. Christensen, and M. van Hecke. Flexible mechanical metamaterials. Nature Reviews Materials, 2 , 2017.

P. Betsch and P. Steinmann. Frame-indifferent beam finite elements based upon the geometrically exact beam theory. International Journal for Numerical Methods in Engineering, 54:1775-1788, 2002.

M. A. Biot. Theory of stress-strain relations in anisotropic viscoelasticity and relaxation phenomena. Journal of Applied Physics, 25:1385-1391, 1954.

A. Cardona and M. Geradin. A beam finite element non-linear theory with finite rotations. International Journal for Numerical Methods in Engineering, 26:2403-2438, 1988.

C. Coulais, J. T. B. Overvelde, L. A. Lubbers, K. Bertoldi, and M. van Hecke. Discontinuous buckling of wide beams and metabeams. Phys. Rev. Lett., 115:044301, 2015.

M. A. Crisfield. A consistent co-rotational formulation for non-linear, three-dimensional, beam-elements. Computer Methods in Applied Mechanics and Engineering, 81(2):131 - 150, 1990.

M. A. Crisfield and G. Jelenić. Objectivity of strain measures in the geometrically exact three-dimensional beam theory and its finite-element implementation. Proc. R. Soc. A, 455:11251147, 1998.

Renko de Vries. Evaluating changes of writhe in computer simulations of supercoiled DNA. The Journal of Chemical Physics, 122:064905, 2005.

Z. Ding, O. Weeger, H. J. Qi, and M. L. Dunn. 4d rods: 3d structures via programmable 1d composite rods. Materials 8 design, 137:256-265, 2018.

E. J. Doedel, A. R. Champneys, T. F. Fairgrieve, Y. A. Kuznetsov, B. Sandstede, and X. J. Wang. AUTO-07p: continuation and bifurcation software for ordinary differential equations. See http://indy.cs.concordia.ca/auto/, 2007.

S. R. Eugster, C. Hesch, P. Betsch, and C. Glocker. Director-based beam finite elements relying on the geometrically exact beam theory formulated in skew coordinates. International Journal for Numerical Methods in Engineering, 97:111-119, 2014.

F. B. Fuller. The writhing number of a space curve. Proc. Natl. Acad. Sci. U.S.A., 68:815-819, 1971.

F. B. Fuller. Decomposition of the linking number of a closed ribbon : A problem from molecular biology. Proc. Natl. Acad. Sci. U.S.A., 75:3557-3561, 1978.

M. Gazzola, L. H. Dudte, A. G. McCormick, and L. Mahadevan. Forward and inverse problems in the mechanics of soft filaments. Royal Society Open Science, 5, 2018.

A. S. Gladman, E. A. Matsumoto, R. G. Nuzzo, L. Mahdevan, and J. A. Lewis. Biomimetic 4D printing. Nature Materials, 15:413-419, 2016.

M. Gomez, D. E. Moulton, and D. Vella. Critical slowing down in purely elastic 'snap-through' instabilities. Nature Physics, $13: 142-145,2017$.

M. Gomez, D. E. Moulton, and D. Vella. Dynamics of viscoelastic snap-through. Journal of the Mechanics and Physics of Solids, 124:781-813, 2019.

B. Halphen and Q. S. Nguyen. Sur les matériaux standards généralisés. Journal de Mécanique, 14:39-63, 1975.

N. Hu and R. Burgueño. Buckling-induced smart applications: recent advances and trends. Smart Materials and Structures, 24:063001, 2015.

A. Ibrahimbegović. On finite element implementation of geometrically nonlinear Reissner's beam theory: three-dimensional curved beam elements. Computer Methods in Applied Mechanics and Engineering, 122:11-26, 1995.

J. A. Jackson, M. C. Messner, N. A. Dudukovic, W. L. Smith, L. Bekker, B. Moran, A. M. Bolobic, A. J. Pascall, E. B. Duoss, K. J. Loh, and C. M. Spadaccini. Field responsive mechanical metamaterials. Science Advances, 4, 2018.

M. K. Jawed, F. Da, J. Joo, E. Grinspun, and P. Reis. Coiling of elastic rods on rigid substrates. PNAS, to appear, 2014.

M.K. Jawed, A.fEugst Novelia, and O.M. OReilly. A Primer on the Kinematics of Discrete Elastic Rods. Springer, 2017.

P. Jung, S. Leyendecker, J. Linn, and M. Ortiz. A discrete mechanics approach to the cosserat rod theory - part 1: static equilibria. International Journal for Numerical Methods in Engineering, 85:31-60, 2011. 
Danny M. Kaufman, R. Tamstorf, B. Smith, J.-M. Aubry, and E. Grinspun. Adaptive nonlinearity for collisions in complex rod assemblies. ACM Trans. on Graphics (SIGGRAPH 2014), 2014.

H. Lang, J. Linn, and M. Arnold. Multi-body dynamics simulation of geometrically exact cosserat rods. Multibody System Dynamics, 25(3):285-312, 2011.

M. Le Merrer, J. Seiwert, D Quéré, and C. Clanet. Shapes of hanging viscous filaments. Europhysics Letters, 84:56004, 2008.

J. Linn. Discrete cosserat rod kinematics constructed on the basis of the difference geometry of framed curvespart i: Discrete cosserat curves on a staggered grid. Journal of Elasticity, pages 1-60, 2019.

J. Linn, H. Lang, and A. Tuganov. Geometrically exact cosserat rods with kelvin-voigt type viscous damping. Mechanical Sciences, 4(1):79-96, 2013.

J. T. Miller, A. Lazarus, B. Audoly, and P. M. Reis. Shapes of a suspended curly hair. Physical Review Letters, 112:068103, 2014.

M. Ortiz and L. Stainier. The variational formulation of viscoplastic constitutive updates. Computer Methods in Applied Mechanics and Engineering, 171:419-444, 1999.

M. R. Shankar, M. L. Smith, V. P. Tondiglia, K. M. Lee, M. E. McConney, D. H. Wang, L-S. Tan, and T. J. White. Contactless, photoinitiated snap-through in azobenzene-functionalized polymers. PNAS, 110:18792-18797, 2013.

Z. Shen, J. Huang, W. Chen, and H. Bao. Geometrically exact simulation of inextensible ribbon. Computer Graphics Forum, $34: 145-154,2015$.

J. C. Simo. A finite strain beam formulation. the three-dimensional dynamic problem. Part I. Computer Methods in Applied Mechanics and Engineering, 49:55-70, 1985.

J. C. Simo and L. Vu-Quoc. A three-dimensional finite-strain rod model. Part II: Computational aspects. Computer Methods in Applied Mechanics and Engineering, 58:79-116, 1986.

V. Sonneville, A. Cardona, and O. Brüls. Geometrically exact beam finite element formulated on the special euclidean group SE(3). Computational Methods Appl. Mech. Engrg., 268:451-474, 2014.

M. Wagner, T. Chen, and K. Shea. Large shape transforming 4D auxetic structures. 3D Printing and Additive Manufacturing, 4:133-141, 2017. 